\title{
FRACTAL DRUM, INVERSE SPECTRAL PROBLEMS FOR ELLIPTIC OPERATORS AND A PARTIAL RESOLUTION OF THE WEYL-BERRY CONJECTURE
}

\author{
MICHEL L. LAPIDUS \\ Dedicated to Professor Gustave Choquet, with sincere respect and admiration
}

\begin{abstract}
Let $\Omega$ be a bounded open set of $\mathbb{R}^{n}(n \geq 1)$ with "fractal" boundary $\Gamma$. We extend Hermann Weyl's classical theorem by establishing a precise remainder estimate for the asymptotics of the eigenvalues of positive elliptic operators of order $2 m(m \geq 1)$ on $\Omega$. We consider both Dirichlet and Neumann boundary conditions. Our estimate-which is expressed in terms of the Minkowski rather than the Hausdorff dimension of $\Gamma$-specifies and partially solves the Weyl-Berry conjecture for the eigenvalues of the Laplacian. Berry's conjecture-which extends to "fractals" Weyl's conjecture-is closely related to Kac's question "Can one hear the shape of a drum?"; further, it has significant physical applications, for example to the scattering of waves by "fractal" surfaces or the study of porous media. We also deduce from our results new remainder estimates for the asymptotics of the associated "partition function" (or trace of the heat semigroup). In addition, we provide examples showing that our remainder estimates are sharp in every possible "fractal" (i.e., Minkowski) dimension.

The techniques used in this paper belong to the theory of partial differential equations, the calculus of variations, approximation theory and-to a lesser extent-geometric measure theory. An interesting aspect of this work is that it establishes new connections between spectral and "fractal" geometry.
\end{abstract}

\section{INTRODUCTION}

The object of this paper is to provide a partial resolution of the Weyl-Berry conjecture for the eigenvalues of the Laplacian on a bounded domain with "fractal" boundary. This conjecture has many significant physical applications, including for example to the scattering of waves by "fractal" surfaces or to the study of porous media.

Received by the editors May 25, 1988 and, in revised form, April 28, 1989.

1980 Mathematics Subject Classification (1985 Revision). Primary 35J20, 35J35, 35P20, 31A25, 31B20, 28A12; Secondary 26B15, 28A75, 31A15, 31A35, 31B35, 41A46, 47A70, 78A40, 78 A45.

Key words and phrases. Dirichlet and Neumann Laplacians, higher order elliptic operators, variational boundary value problems, inverse problems, spectral theory, approximation theory, asymptotics of eigenvalues, remainder estimate, Weyl-Berry conjecture, fractals, Minkowski and Hausdorff dimensions, vibrations of fractal drums, spectral and fractal geometry.

The author's research was partially supported by the National Science Foundation under Grant DMS-8703138. 
We shall consider in this work the cases of both Dirichlet and Neumann boundary conditions, as well as of higher order positive elliptic operators (with locally constant leading coefficients). For the sake of clarity, however, we will first discuss in this introduction the simpler and more familiar case of the Dirichlet Laplacian.

Weyl's asymptotic formula for the Dirichlet Laplacian. Let $\Omega$ be an arbitrary nonempty bounded open set in $\mathbb{R}^{n} \quad(n \geq 1)$, with boundary $\Gamma:=\partial \Omega$. We consider the following eigenvalue problem:

$$
\left\{\begin{aligned}
-\Delta u=\lambda u & \text { in } \Omega, \\
u=0 & \text { on } \Gamma,
\end{aligned}\right.
$$

where $\Delta=\sum_{k=1}^{n} \partial^{2} / \partial x_{k}^{2}$ denotes the Dirichlet Laplacian in $\Omega$.

We interpret $(P)$ in the variational sense. More precisely, the scalar $\lambda$ is said to be an eigenvalue of the Dirichlet problem (P) if there exists $u \neq 0$ in $H_{0}^{1}(\Omega)$ [the completion of $C_{0}^{\infty}(\Omega)$, the space of smooth functions with compact support in $\Omega$, with respect to the Sobolev norm $\left.\|\cdot\|_{H^{1}(\Omega)}\right]$ satisfying $-\Delta u=\lambda u$ in the distributional sense.

It is classical that the spectrum of $(\mathrm{P})$ is discrete and is composed of an infinite sequence of positive eigenvalues, written in increasing order according to their multiplicity:

$$
0<\lambda_{1} \leq \lambda_{2} \leq \cdots \leq \lambda_{i} \leq \cdots, \quad \text { with } \lambda_{i} \rightarrow+\infty \text { as } i \rightarrow \infty .
$$

As is well known, problem (P) can be considered as a mathematical model for the study of the (steady-states) vibrations of a drum. Indeed, the natural frequencies (or "normal modes") of a vibrating membrane are proportional to the square root of the eigenvalues $\lambda_{i}$; further, the lowest frequency is called the fundamental tone and the higher frequencies are called the overtones of the drum. In this paper, we shall be primarily interested in the case when $\Gamma$ is very irregular; i.e., that of a "drum with fractal boundary".

In 1911, Hermann Weyl [We1, 2]-thereby solving an outstanding problem posed in 1905 by the physicist $\mathrm{H}$. A. Lorentz about the asymptotics of the high frequency modes of musical instruments (see [Ka, pp. 3-4])-showed that

$$
\lambda_{i} \sim C_{n}\left(i /|\Omega|_{n}\right)^{2 / n} \text { as } i \rightarrow \infty,
$$

where the "classical constant" $C_{n}=(2 \pi)^{2}\left(\mathscr{B}_{n}\right)^{-2 / n}$ depends only on $n$. (See also [CoHi, $\mathrm{Ka}, \mathrm{ReSi}, \mathrm{Si}, \mathrm{Mt} 3, \mathrm{Ho} 2]$ etc.) [Here, $|A|_{n}$ denotes the $n$-dimensional Lebesgue measure or "volume" of $A \subset \mathbb{R}^{n}$ and $\mathscr{B}_{n}$ is the volume of the unit ball in $\mathbb{R}^{n}$; further, the symbol " " means that the ratio of left and right sides of (1.2) tends to one.]

The asymptotic behavior of the eigenvalues $\left\{\lambda_{i}\right\}_{i=1}^{\infty}$ can also be deduced from that of the "counting function" $N(\lambda)$, the number of positive eigenvalues (counted with multiplicity) $\leq \lambda$ :

$$
N(\lambda):=\#\left\{i \geq 1: 0<\lambda_{i} \leq \lambda\right\} \text { for } \lambda>0 .
$$


In fact, since $N\left(\lambda_{i}\right)=i$, Weyl's famous asymptotic formula (1.2) can be equivalently stated as follows:

$$
N(\lambda) \sim(2 \pi)^{-n} \mathscr{B}_{n}|\Omega|_{n} \lambda^{n / 2} \quad \text { as } \lambda \rightarrow+\infty .
$$

This result-initially obtained by Weyl for sufficiently regular domains-has since been generalized in many different ways. (See, e.g., [Ag, ReSi, Si, Ho2, La1, 2, FlLa1, 2, Ya] and the references therein.) In the present case of the Dirichlet Laplacian, it is now known to hold for an arbitrary bounded open set in $\mathbb{R}^{n}$ [Mt1-3].

Kac's inverse spectral problem. In a beautiful paper, entitled "Can one hear the shape of a drum?", Mark Kac [Ka] asked the following question: Can someone with perfect pitch recover the precise shape of a drum just by listening to its fundamental tone and all the overtones? This question-in conjunction with Weyl's result (1.4) - has motivated numerous works on the subject during the past twenty years. (See, e.g., the review papers [Pr and Ya, §1].) Recently, Urakawa [Ur] has discovered two isospectral domains of $\mathbb{R}^{n}(n \geq 4)$ which are not isometric. Consequently, Kac's inverse problem does not have an affirmative answer in general. However, it is known that one can recover a lot of geometric information about $\Omega$ from the spectrum of $(\mathrm{P})$ : for instance, the volume $|\Omega|_{n}$ [according to Weyl's formula (1.4)] and, for a smooth domain, the curvature of the boundary $\Gamma$ [McKean and Singer [McSn]]. Accordingly, it is natural to wonder whether one could "hear" (some other) "shape(s)" of the boundary: for example, the "surface" $|\Gamma|_{n-1}$ (i.e., the $(n-1)$-dimensional volume of $\Gamma$ ), or possibly, if the boundary is not smooth, the "fractal" dimension of $\Gamma$. We will now address certain aspects of this inverse spectral problem.

The conjectures of Weyl and Berry. Actually, if the boundary $\Gamma$ is smooth (i.e., of class $C^{\infty}$ ), it is even known that Weyl's asymptotic formula (1.4) can be extended as follows:

$$
N(\lambda)=(2 \pi)^{-n} \mathscr{B}_{n}|\Omega|_{n} \lambda^{n / 2}+O\left(\lambda^{(n-1) / 2}\right) \quad \text { as } \lambda \rightarrow+\infty .
$$

This result was first obtained (in this context) by Seeley [Se1, 2] when $n=3$ and later generalized to arbitrary $n \geq 1$ by Pham The Lai [Ph]. Its proof (as well as aspects of that of [Iv1,2] below) makes use of techniques from the theory of spectral transforms and of Fourier integral operators, originating in a closely related work of Hörmander [Ho1]. [See also [Ho2, Vol. III, §XVII.5] and the relevant references therein.]

The remainder estimate (1.5) constitutes an important step on the way to Weyl's conjecture [We3] which states that if $\Gamma$ is sufficiently "smooth", then the asymptotic expansion of $N(\lambda)$ admits a second term, proportional to $\lambda^{(n-1) / 2}$

Recently, Ivrii [Iv1, 2] made great progress towards the resolution of this conjecture; he showed that if $\Omega$ is a bounded domain with $C^{\infty}$ boundary $\Gamma$ (and if the manifold $\bar{\Omega}$ does not have too many multiply reflected closed 
geodesics), then the following remarkable result holds:

(1.6) $N(\lambda)=(2 \pi)^{-n} \mathscr{B}_{n}|\Omega|_{n} \lambda^{n / 2}-c_{n}|\Gamma|_{n-1} \lambda^{(n-1) / 2}+o\left(\lambda^{(n-1) / 2}\right) \quad$ as $\lambda \rightarrow+\infty$, where $c_{n}$ is a positive constant depending only on $n$. [See also Kuznetsov [Ku] for a simple case of (1.6) and especially Melrose [Ms1, 2]. Further, for an exposition of the proof of Ivrii's theorem, the reader may also wish to consult Hörmander's treatise [Ho2, Vol. III, §XVII.3 and Vol. IV, §XXIX.3] where are combined Ivrii's wave equation method and Melrose's simplifications based on results on the propagation of singularities.] We note that Weyl's conjecture is known to fail in some cases. (See [Gr, Bd].)

In 1979, the physicist Michael V. Berry [Be1, 2]-motivated in part by the study of the scattering of light by random surfaces-extended Weyl's conjecture to the "fractal" case. He conjectured that if $\Omega$ has a "fractal" boundary $\Gamma$ with Hausdorff dimension $H \in(n-1, n]$, then

$$
N(\lambda)=(2 \pi)^{-n} \mathscr{B}_{n}|\Omega|_{n} \lambda^{n / 2}-c_{n, H^{\prime}} \mathscr{H}_{H}(\Gamma) \lambda^{H / 2}+o\left(\lambda^{H / 2}\right) \quad \text { as } \lambda \rightarrow+\infty,
$$

where $c_{n, H}$ is a positive constant depending only on $n$ and $H$, and $\mathscr{H}_{H}(\Gamma)$ denotes the $H$-dimensional Hausdorff measure of $\Gamma$.

Observe that if $\Gamma$ is sufficiently smooth (e.g., of class $C^{1}$ ), then $H=n-1$ and (1.7) reduces to (1.6). In general, however, $\Gamma$ may be extremely irregular and hence $H$ is a real number $>n-1$.

Actually, Berry's conjecture, as stated above in terms of the Hausdorff dimension, is not correct. Indeed, in an important work, Brossard and Carmona [BrCa] have recently constructed a simple counterexample to (1.7) and suggested that $H$ should be replaced by $D$, the Minkowski dimension of $\Gamma$. Under suitable assumptions, they also obtained one and two-sided pre-Tauberian estimates (expressed in terms of $D$ ) for the second term in the asymptotic expansion of the "partition function" $Z(t):=\sum_{i=1}^{\infty} e^{-\lambda_{t} t}$, a well known regularization of the "counting function" $N(\lambda)$. (See [BrCa, §3].) That the less familiar $D$ (Minkowski) should be substituted for $H$ (Hausdorff) in (1.7) is also clear $a$ posteriori in light of the paper by Fleckinger and Lapidus [FlLa2] on eigenvalue problems with indefinite weights, in conjunction with the present work. [See especially $\S 3$ below (in particular, Proposition 3.1 and its Corollaries 3.1 and 3.3) where are provided several results connecting the Minkowski dimension of $\Gamma$ and the "tessellations" of $\mathbb{R}^{n}$ into small cubes, as well as Examples 5.1-5.1' .]

In this paper, we make a significant step towards the resolution of the (modified) Weyl-Berry conjecture by obtaining a remainder estimate associated to Weyl's asymptotic formula (1.4), valid even if the boundary $\Gamma$ is very irregular. More precisely, we show that if $\Gamma$ is "fractal" (i.e., if the Minkowski dimension $D$ of $\Gamma$ lies in $(n-1, n])$, then, for all $d>D$,

$$
N(\lambda)=(2 \pi)^{-n} \mathscr{B}_{n}|\Omega|_{n} \lambda^{n / 2}+O\left(\lambda^{d / 2}\right) \text { as } \lambda \rightarrow+\infty ;
$$

furthermore, except possibly in the degenerate case when the upper Minkowski content $\mathscr{M}_{D}$ of $\Gamma$ is infinite, (1.8) also holds if $d=D$. (See Definition 1.1 below and $\S 2.1$ for the definitions of $D$ and $\mathscr{M}_{D}$. ) 
Observe that this result is a counterpart in the "fractal case" of the remainder estimate (1.5), valid in the "smooth case" and improved successively by Hörmander, Seeley, and Pham The Lai (among others).

Moreover, we prove that the same result holds for the Neumann problem if, in addition, $\Gamma$ satisfies the " $\left(C^{\prime}\right)$ condition" (see Definition 2.2$)$; this is the case, for example, if $\Omega$ obeys a "segment condition" [Ag, p. 11] or, loosely speaking, if the boundary $\Gamma$ is not "too long". Actually, if the " $\left(C^{\prime}\right)$ condition" is not satisfied, then even the leading asymptotics of $N(\lambda)$ need not be given by Weyl's formula (1.4) [Mt3, §VII] and hence the remainder estimate (1.8) cannot hold.

We note that Ivrii's theorem (1.6) and the Weyl-Berry conjecture (1.7) do extend to the Neumann problem provided that the second term in the righthand side of (1.6) and (1.7), respectively, is preceded by " + " rather than by "-". In the present case when $\Gamma$ need not be smooth, however, proper care is required to formulate and establish the extension of $(1.8)$ to Neumann boundary conditions: $\partial u / \partial n=0$ on $\Gamma$ (where $\partial / \partial n$ denotes a "normal derivative" along $\Gamma$ ). In particular, we say that $\lambda$ is an eigenvalue of the (variational) Neumann problem if there exists a nonzero $u$ in the Sobolev space $H^{1}(\Omega)$ satisfying the distributional equation $-\Delta u=\lambda u$.

Although it falls short of showing the existence of a second term, our remainder estimate (1.8) is of the desired form and provides good evidence that the (appropriately modified) Weyl-Berry conjecture might be true.

Remarks 1.1. (a) We stress that no assumption of "self-similarity" (or, more generally, "self-alikeness" of any kind), in the sense of Mandelbrot [Md1, 2], has been made about $\Gamma$.

(b) The larger $D$, the more irregular $\Gamma$. Further, we always have $D \in$ $[n-1, n]$ since $\Gamma=\partial \Omega$ and $\Omega \subset \mathbb{R}^{n}$. We will say here that $\Gamma$ is "fractal" if $D \in(n-1, n]$; this is the case in particular if $D$ is noninteger.

(c) Of course, if $\Gamma$ is "smooth" enough (e.g., if $\Gamma$ is $(n-1)$-rectifiable [Fe, pp. 251 and 275] and hence, in particular, if $\Gamma$ is of class $C^{1}$ ), then $H=D=n-1$, the topological dimension of $\Gamma$. In general, however, we have $n-1 \leq H \leq D \leq n$.

(d) Intuitively, the Hausdorff (resp., Minkowski) dimension can be understood as follows: for $\varepsilon>0$, let $\mathscr{N}(\varepsilon)$ be the number of $n$-dimensional cubes (or balls) of diameter $\leq \varepsilon$ (resp., $=\varepsilon$ ) needed to cover $\Gamma$; then, very roughly, if $\mathscr{N}(\varepsilon)$ increases like $\mathscr{N}(\varepsilon) \propto \varepsilon^{-H}$ (resp., $\varepsilon^{-D}$ ), as $\varepsilon \rightarrow 0^{+}$, one says that $\Gamma$ has Hausdorff (resp., Minkowski) dimension $H$ (resp., D). (Further explanation of (b)-(d) is given in $\S 3$.)

(e) If $D=n-1$ (i.e., if $H=D=n-1$ ), then our proof shows that the above remainder estimate still holds provided that the error term $O\left(\lambda^{d / 2}\right)$ is replaced by $O\left(\lambda^{d / 2} \log \lambda\right)$ in (1.8); this last result is a restatement and a slight extension of one obtained by Métivier in [Mt2, 3]. (See also Courant [Co] and Courant and Hilbert [CoHi, $\S$ VI.5, pp. 443-445] for an early special case of this result.) 
(f) We do not consider here, as was done in [Be1, 2], the mathematically ill-defined case when $\Omega$ itself is "fractal" (and hence not open). We intend to tackle this problem in a future work [La10].

The Weyl-Berry conjecture has many significant physical applications, including, for example [Be1, 2], to the scattering of waves by "fractal" surfaces, the study of the vibrations of a "fractal drum" or of water in a lake $[n=2$ and $D \in(1,2)]$, the oscillations of the Earth or the acoustic modes of a concert hall with very irregular walls $[n=3$ and $D \in(2,3)]$. (Of course, Berry was using $H$ instead of $D$ in [Be1, $\S 2$, Class I, p. 51].)

See also the examples of our results in $\$ 5.1$ which could apply, for instance, to the scattering of light from a "triadic Koch island" [Md1, pp. 42-45] in the form of Koch's snowflake curve and to the high frequency modes of a "Koch drum" $(n=2$ and $D=H=\log 4 / \log 3=1.2618 \ldots)$.

The Minkowski dimension. Next, we briefly recall the definition of the Minkowski dimension. We note that the latter-which is sometimes called the Cantor-Minkowski-Bouligand dimension-is closely related to the entropy (or information) dimension of Kolmogorov occurring in the theory of dynamical systems and to the "box dimension" used by the practitioners of "fractal geometry". (See [Bo, KhSa, Fe, Md1, 2, PiTo, Ce, MrVu] etc., as well as Definition 2.1 and $\S 3$.)

Definition 1.1. For $\varepsilon>0$, let $\Gamma_{\varepsilon}$, the $\varepsilon$-neighborhood of $\Gamma$, be the set of $x \in \mathbb{R}^{n}$ within a distance $<\varepsilon$ from $\Gamma$. Let $D=D(\Gamma)$ be the infimum of the positive numbers $d$ such that

$$
\mathscr{M}_{d}=\mathscr{M}_{d}(\Gamma):=\limsup _{\varepsilon \rightarrow 0^{+}} \varepsilon^{-(n-d)}\left|\Gamma_{\varepsilon}\right|_{n}=0 .
$$

Then $D$ (resp., $\mathscr{M}_{D}$ ) is called the Minkowski dimension [resp., ( $D$-dimensional) upper Minkowski content] of $\Gamma$.

We have $\mathscr{M}_{d}=0$ for $d>D$ and $\mathscr{M}_{d}=+\infty$ for $d<D$; moreover, $\mathscr{M}_{D}$ may be infinite or finite (possibly zero). [For most usual "fractals", however, we have $0<\mathscr{M}_{D}<+\infty$.] Thus, by Remark 1.1(b), our partial solution of the Weyl-Berry conjecture [estimate (1.8)] is a direct corollary of the following result:

Theorem 1.1. Let $d \in(n-1, n]$ be such that $\mathscr{M}_{d}(\Gamma)<+\infty$. Then estimate (1.8) holds for this value of $d$.

We point out that our remainder estimate (1.8) is in general "best possible". (See Examples 5.1 and 5.1' , as well as 5.2.)

Moreover, we also deduce corresponding results for the asymptotics as $t \rightarrow 0^{+}$ of the "partition function" (or trace of the heat semigroup)

$$
Z(t):=\int_{0}^{\infty} e^{-\lambda t} d N(\lambda)=\sum_{i=1}^{\infty} e^{-\lambda_{\imath} t}
$$


associated with this problem. In particular, we show for the Dirichlet Laplacian that if $\Omega$ is an arbitrary bounded open set with "fractal" boundary $\Gamma$, then

$$
Z(t)=(4 \pi)^{-n / 2}|\Omega|_{n} t^{-n / 2}+O\left(t^{-D / 2}\right) \quad \text { as } t \rightarrow 0^{+},
$$

provided that $\mathscr{M}_{D}(\Gamma)<+\infty$. [If $\mathscr{M}_{D}(\Gamma)=+\infty$, then we must replace $D$ by $d$, with $d>D$ arbitrarily close to $D$, in the right-hand side of (1.10).] (See Theorem 2.3 and-for the more general case considered below-Theorem 2.2. The special case of the Dirichlet Laplacian when $\mathscr{M}_{D}(\Gamma)<+\infty$ was already obtained (by different methods) in [BrCa].)

Extension to higher order elliptic operators. We also obtain the counterpart of Theorem 1.1 and estimate (1.8) for positive uniformly elliptic operators of order $2 m(m \geq 1): \mathscr{A}=\sum_{|\alpha| \leq m,|\beta| \leq m}(-1)^{|\alpha|} D^{\alpha}\left(a_{\alpha \beta}(x) D^{\beta}\right)$, with (locally) constant leading coefficients and with Dirichlet or Neumann (or more generally, mixed Dirichlet-Neumann) boundary conditions. Hence, in particular, we show how to extend our partial resolution of the Weyl-Berry conjecture-which corresponds to the Laplace operator-to higher order elliptic operators. We refer the reader to $\S 2$ (particularly Theorem 2.1 and its corollaries) for a precise statement of our hypotheses and results in this general case.

Remark 1.2. Actually, in the case of the Dirichlet problem, we prove a somewhat sharper result. We introduce a slightly different notion of "fractal dimension", denoted by $\widetilde{D}$ and called the Minkowski dimension of $\Gamma:=\partial \Omega$, relative to $\Omega$. (See Definition 2.1.) It is obtained in the same way as $D$ in Definition 1.1 except that $\Gamma_{\varepsilon}$ is replaced by $\widetilde{\Gamma}_{\varepsilon}:=\Gamma_{\varepsilon} \cap \Omega=\{x \in \Omega: d(x, \Gamma)<\varepsilon\}$, the (one-sided) $\varepsilon$-neighborhood of $\Gamma$ relative to $\Omega$. By construction, $\widetilde{D} \leq D$; further, for the Dirichlet problem, Theorem 1.1 and estimates (1.8) and (1.10), as well as their counterpart for higher order operators, also hold with $D$ replaced by $\widetilde{D}$ and $\mathscr{M}_{d}$ by the corresponding $\widetilde{\mathscr{M}}_{d}$. (See Theorems $2.1-2.3$ and Corollary 2.1.)

It is noteworthy that, according to (the analogue of) Remark 1.1(e), different estimates hold in the "fractal" case $(D \in(n-1, n])$ and the "nonfractal" case ( $D=H=n-1$, but $\Gamma$ is not necessarily smooth). Somewhat paradoxically, this would seem to indicate (in the special case of the Laplacian) that a "drum with fractal boundary" usually plays more regularly than a more "standard drum" (one with "nonfractal" but yet irregular boundary).

Our proof of Theorem 2.1 (the counterpart of Theorem 1.1) is purely analytic. In particular, we do not make use of probabilistic results. Partially motivated by [FlLa2], it extends to the "fractal" case that of [CoHi and $\mathrm{Mt} 2,3$ ] . Variational techniques-based on the "max-min formula" for the $i$ th eigenvalue and its consequences-play an essential role; among them, we mention the method of " $i$-width" in Sobolev spaces coming from approximation theory [Lo, BiSo, BG, Ek, Pn], and the method known by mathematical physicists as the "DirichletNeumann bracketing" [CoHi, ReSi, Mt1-3, La1-3, FlLa 1, 2]. We use finer and finer "tessellations" of $\mathbb{R}^{n}$ into small cubes (Whitney-type coverings); more 
precisely, we "exhaust" $\Omega$ by cubes whose size tends to zero as you approach $\Gamma$. This enables us to detect-from the point of view of harmonic analysis and spectral theory-the influence of $\Omega$ and especially of the irregularities (or "fractality") of its boundary $\Gamma$. Naturally, in order to keep the problem under firm control, it is crucial, in particular, to obtain precise estimates in terms of the Minkowski dimension of $\Gamma$ for certain "counting functions" near the boundary. (See Proposition 4.6.) Curiously enough, in our present proof, the aforementioned dichotomy between the spectral behavior in the "fractal" and the "nonfractal" cases, respectively (see, especially, Theorem 2.1 as well as Corollaries 2.1 and 2.2), can be attributed to the following elementary fact: the partial sums of a geometric series of ratio $2^{\theta}$ take different forms according to whether $\theta \neq 0$ or $\theta=0$, respectively. [See Remarks 4.10 and 4.11(b); here, $\theta:=\widetilde{D}-(n-1)$ (resp., $:=D-(n-1))$ for the Dirichlet (resp., Neumann) problem.]

In classical spectral geometry (e.g., [C, GuKz, Iv1, 2, Ms1, 2, OsWi, Ph, Se1, 2], and relevant references therein), one works mostly within the framework of smooth (Riemannian) manifolds. Furthermore, in geometric measure theory (e.g., [Al, Fe]), one extends the classical methods of the calculus of variations as well as parts of differential geometry in order to deal with generalized surfaces (currents, varifolds, etc.) which need not be smooth but are essentially of integral (Hausdorff) dimension. An interesting aspect of the present paper is that it goes beyond the traditional areas of investigation and establishes in the process new connections between spectral and "fractal" geometry.

Because our work draws on several fields of mathematics which may not be all familiar to the reader-elliptic partial differential equations, calculus of variations, spectral theory, approximation theory, as well as aspects of geometric measure theory and "fractal geometry"-we have endeavored to supply some background material whenever possible.

We close this introduction by indicating how the rest of the paper is organized:

After having presented the necessary notation and definitions of $\S 2.1$, we give precise statements of our hypotheses and main results (Theorems 2.1 and 2.3 together with Corollaries 2.1 and 2.2) in $\S 2.2$. Further, we deduce from our remainder estimates for the "counting function" $N(\lambda)$, as $\lambda \rightarrow+\infty$, a corresponding one for the "partition function" (or trace of the heat semigroup) $Z(t)$, as $t \rightarrow 0^{+}$. (See Theorems 2.2 and 2.3.)

In $\S 3$, we discuss some basic properties of the Minkowski dimension; we also briefly explain why the Minkowski dimension $D$ should be better suited to the study of spectral theory or (aspects of) harmonic analysis than the more familiar Hausdorff dimension $H$. In addition, theoretical reasons for favoring $D$ over $H$ or other "fractal dimensions" are given throughout this paper.

The proofs of our main results are given in $\S 4$. After having provided some background and preliminary estimates, we establish Theorem 2.1 (the generalization of Theorem 1.1) in $\S 4.2$, both for Dirichlet and Neumann boundary 
conditions. The techniques and ideas developed here should be useful in later work dealing with both spectral and "fractal" geometry. We have tried to present a proof that was essentially self-contained, especially for the Dirichlet problem. However, some readers may wish to consult $\S 4$ only briefly on a first reading.

Towards the end of $\S 4$, we also present some extensions of our results for the Neumann problem, valid, for example, for "quasidisks" and their higher dimensional analogues ("Jones domains"). (See Theorem 4.1.)

We illustrate our results in $\S 5.1$ by applying them to several concrete examples of mathematical or of physical interest. This enables us, in particular, to show that-in the "fractal" case-our remainder estimates are optimal in every possible "fractal" (i.e., Minkowski) dimension; more precisely, we construct a one-parameter family of examples for which our remainder estimates are sharp and, as the parameter varies, the Minkowski dimension $D$ of $\Gamma$ takes on every value in $(n-1, n)$ whereas $H \equiv n-1$. (See Examples 5.1-5.1' .) [Berry's original conjecture-expressed in terms of the Hausdorff dimension $H$ of $\Gamma$ obviously fails for Examples 5.1 and $5.1^{\prime}$.] Finally, we also propose several open problems and a conjecture; the latter-stated in $\$ 5.2$-extends and modifies the Weyl-Berry conjecture.

\section{NOTATION AND MAIN RESULTS}

2.1. Notation and definitions. Throughout this paper, we shall use the following notation:

Let $m, n$ be integers $\geq 1$. Let $\Omega$ be an arbitrary (nonempty) bounded open subset of $\mathbb{R}^{n}$, with (topological) boundary $\Gamma:=\partial \boldsymbol{\Omega}$.

The interior (resp., closure, boundary) of $A \subset \mathbb{R}^{n}$ is denoted by Int $A$ (resp., $\bar{A}, \partial A$ ). If, in addition, $A$ is (Lebesgue) measurable, we denote by $|A|_{n}$-or simply $|A|$ when no ambiguity will result-its $n$-dimensional Lebesgue measure or "volume". (See, e.g., [Cn, p. 21, or Fe].)

For $x \in \mathbb{R}^{n}$ and $A \subset \mathbb{R}^{n}, d(x, A):=\inf \{|x-y|: y \in A\}$ denotes the Euclidean distance from $x$ to $A$; here, $|x|=\left(\sum_{k=1}^{n} x_{k}^{2}\right)^{1 / 2}$ is the Euclidean norm of $x=\left(x_{1}, \ldots, x_{n}\right)$. Given $\varepsilon>0, A_{\varepsilon}$ denotes the $\varepsilon$-neighborhood (or open tubular neighborhood of radius $\varepsilon$ ) of $A \subset \mathbb{R}^{n}$ :

$$
A_{\varepsilon}:=\left\{x \in \mathbb{R}^{n}: d(x, A)<\varepsilon\right\} .
$$

If $I$ is a subset of $J, J \backslash I$ stands for the complement of $I$ in $J$. If $J$ is a finite set, we let \#J be its cardinality.

Given $\alpha=\left(\alpha_{1}, \ldots, \alpha_{n}\right) \in \mathbb{N}^{n}, D^{\alpha}$ stands for the derivative of order $|\alpha|:=$ $\alpha_{1}+\cdots+\alpha_{n}: D^{\alpha}:=\partial^{|\alpha|} / \partial x_{1}^{\alpha_{1}} \cdots \partial x_{n}^{\alpha_{n}} ;$ moreover, for $\xi=\left(\xi_{1}, \ldots, \xi_{n}\right) \in \mathbb{R}^{n}$, we set $\xi^{\alpha}=\xi_{1}^{\alpha_{1}} \cdots \xi_{n}^{\alpha_{n}}$.

As usual, $L^{2}(\Omega)$ is the space of all (complex-valued) functions $u$ which are square-integrable with respect to $n$-dimensional Lebesgue measure $|\cdot|_{n}$ (also denoted $d x$ ); the Hilbert space $L^{2}(\Omega)$ is equipped with its natural norm $\|\cdot\|_{L^{2}(\Omega)}$ and inner product $(\cdot, \cdot)_{L^{2}(\Omega)}$. For $p \in \mathbb{N}, C^{p}(\Omega)$ is the space of $p$ times continuously differentiable functions on $\Omega$. 
By $H^{m}(\Omega)$ we mean the Sobolev space of all (complex-valued) functions $u \in L^{2}(\Omega)$ with distributional derivatives $D^{\alpha} u$ also in $L^{2}(\Omega)$ for $|\alpha| \leq m$. Recall that $H^{m}(\Omega)$ is a Hilbert space when endowed with the norm

$$
\|u\|_{H^{m}(\omega)}=\left(\int_{\Omega} \sum_{|\alpha| \leq m}\left|D^{\alpha} u(x)\right|^{2} d x\right)^{1 / 2}=\left(\sum_{|\alpha| \leq m}\left\|D^{\alpha} u\right\|_{L^{2}(\Omega)}^{2}\right)^{1 / 2} .
$$

Further, $H_{0}^{m}(\Omega)$ is the closed subspace of $H^{m}(\Omega)$ obtained by completing $C_{0}^{\infty}(\Omega)$ with respect to this norm; of course, $H_{0}^{m}(\Omega)$ is also a Hilbert space for $\|\cdot\|_{H^{\prime}(\Omega)}$. (See, e.g., [Ad, Ag, LiMa].) Here, $C_{0}^{\infty}(\Omega)$ stands for the space of infinitely differentiable functions with compact support contained in $\Omega$. [When necessary, similar notation will be used for functions defined on other open subsets of $\mathbb{R}^{n}$.]

Let $f, g$ be real-valued functions on $(0,+\infty)$, with $g \geq 0$. We write $f(\lambda)=O(g(\lambda))$, as $\lambda \rightarrow+\infty$, if there exist positive constants $C$ and $\lambda_{0}$ such that $|f(\lambda)| \leq C g(\lambda), \forall \lambda \geq \lambda_{0}$. Moreover, we write $f(\lambda) \approx g(\lambda)$, as $\lambda \rightarrow+\infty$, whenever there exist positive constants $c_{1}, c_{2}$, and $\lambda_{0}$ such that $c_{1} g(\lambda) \leq$ $f(\lambda) \leq c_{2} g(\lambda), \forall \lambda \geq \lambda_{0}$.

We shall use various constants throughout the text; they will be denoted by $c, c_{0}, c^{\prime}, C$, etc. Often, the same letter will be used to represent different constants.

In order to state our results, we shall need the following definitions. The "fractal dimension" $\widetilde{D}$ (resp., $D$ ) defined below will be used to study Dirichlet (resp., Neumann or, more generally, mixed) boundary conditions.

Definition 2.1. (a) Given $\varepsilon>0$, let $\Gamma_{\varepsilon}=\left\{x \in \mathbb{R}^{n}: d(x, \Gamma)<\varepsilon\right\}$ be the $\varepsilon$-neighborhood of $\Gamma$, as in (2.1). For $d \geq 0$, let

$$
\mathscr{M}_{d}=\mathscr{M}_{d}(\Gamma):=\limsup _{\varepsilon \rightarrow 0^{+}} \varepsilon^{-(n-d)}\left|\Gamma_{\varepsilon}\right|_{n}
$$

be the $d$-dimensional upper Minkowski content of $\Gamma$. Then

$$
D=D(\Gamma):=\inf \left\{d \geq 0: \mathscr{M}_{d}(\Gamma)=0\right\}=\sup \left\{d \geq 0: \mathscr{M}_{d}(\Gamma)=+\infty\right\}
$$

is called the Minkowski dimension of $\Gamma$.

(b) Given $\varepsilon>0$, let

$$
\widetilde{\Gamma}_{\varepsilon}:=\Gamma_{\varepsilon} \cap \Omega=\{x \in \Omega: d(x, \Gamma)<\varepsilon\}
$$

be the one-sided $\varepsilon$-neighborhood (or one-sided tubular neighborhood) of $\Gamma$. For $d \geq 0$, let

$$
\widetilde{\mathscr{M}}_{d}=\widetilde{\mathscr{M}}_{d}(\Gamma):=\limsup _{\varepsilon \rightarrow 0^{+}} \varepsilon^{-(n-d)}\left|\widetilde{\Gamma}_{\varepsilon}\right|_{n}
$$

be the $d$-dimensional upper Minkowski content of $\Gamma$, relative to $\Omega$. Then

$$
\widetilde{D}=\widetilde{D}(\Gamma):=\inf \left\{d \geq 0: \mathscr{M}_{d}(\Gamma)=0\right\}=\sup \left\{d \geq 0: \mathscr{M}_{d}(\Gamma)=+\infty\right\}
$$

is called the Minkowski dimension of $\Gamma$, relative to $\Omega$. 
Moreover, if in (a), $0<\mathscr{M}_{D}<+\infty$ and the upper limit in (2.3) can be replaced by a true limit (as $\varepsilon \rightarrow 0^{+}$), with $d:=D$, then $\Gamma$ is said to be Minkowski "measurable" (or "contented") and $\mathscr{M}_{D}$ is called the Minkowski "measure" (or "content") of $\Gamma$. [In (b), one defines similarly the Minkowski "measurability" and "measure" of $\Gamma$, relative to $\Omega$.]

Remarks 2.1. (a) Clearly, $\Gamma_{\varepsilon}$ and $\widetilde{\Gamma}_{\varepsilon}$ are bounded open subsets of $\mathbb{R}^{n}$; further, since $\widetilde{\Gamma}_{\varepsilon} \subset \Gamma_{\varepsilon}$, we have $\widetilde{D} \leq D$. Moreover, $n-1 \leq D \leq n$. [The fact that $D \geq n-1$ will be justified in Corollary 3.2 below.]

(b) By construction, we have $\mathscr{M}_{d}=+\infty$ if $d<D$ and $\mathscr{M}_{d}=0<+\infty$ if $d>D$. Moreover, $\mathscr{M}_{D} \in[0,+\infty]$. [For most of the classical "fractals" studied for instance in [Ce, Fa, Md1, 2], however, we have $0<\mathscr{M}_{D}<+\infty$.] Further, if $0<\mathscr{M}_{d}<+\infty$ for some $d \geq 0$, then we must have $d=D$. [The same remark holds with $\mathscr{M}_{d}$ replaced by $\widetilde{\mathscr{M}}_{d}$ and $D$ by $\widetilde{D}$.]

(c) It is easy to check that the last equality in (2.4) holds. Indeed, given $d<D$, choose $t \in(d, D)$; by definition, $\mathscr{M}_{t} \in(0,+\infty]$ and thus, by $(2.3)$, $\mathscr{M}_{d}=+\infty$. Similarly, given $d>D$, there exists $t \in(D, d)$ such that $\mathscr{M}_{t}=0$; and hence; by (2.3), $\mathscr{M}_{d}=0$. [An identical argument applies to (2.7).]

(d) The "critical exponent" $\widetilde{D}$ (resp., $D$ ) will serve as a measure of the roughness of the boundary $\Gamma$ in the Dirichlet (resp., Neumann) problem: the larger $\widetilde{D}$ (resp., $D$ ), the more irregular $\Gamma$.

(e) The notion of Minkowski dimension was introduced by Bouligand in [Bo]; many of its properties can be found in [Ce, $\mathrm{Fe}, \mathrm{Md} 1,2, \mathrm{MrVu}$ ], as well as $\S 3$ below. Naturally, one can define-exactly as in Definition $2.1(\mathrm{a})-D(A)$ and $\mathscr{M}_{D}(A)$ for any subset $A \subset \mathbb{R}^{n}$; clearly, $D(A)$ increases with $A$ and if $A$ is bounded, $0 \leq D(A) \leq n$. [One can also define-by analogy with Definition 2.1(b)-the Minkowski dimension and content of $A$, relative to $B \subset \mathbb{R}^{n}$; of course, if $A$ or $B$ is not (Lebesgue) measurable, we must interpret $|\cdot|_{n}$ as denoting the outer Lebesgue measure.]

In the following, unless otherwise specified, we shall use the notation of Definition 2.1 and write in particular:

$$
D=D(\Gamma) \text { and } \widetilde{D}=\widetilde{D}(\Gamma) .
$$

The following definition will be needed only in the case of mixed DirichletNeumann boundary conditions. For simplicity, we adopt the definition from [Mt2, p. 15] rather than the slightly different one from [Mt3, pp. 154 and 156]. (See, however, Remark 2.4(f) below.)

Definition 2.2. The open set $\Omega$ satisfies the " $\left(C^{\prime}\right)$ condition" if there exist positive constants $\varepsilon_{0}, M$, and $t_{0}$, with $\varepsilon_{0} M<t_{0}$, an open cover $\left\{\Omega_{j}\right\}_{j=1}^{N}$ of $\widetilde{\Gamma}_{\varepsilon_{0}}$, and nonzero vectors $h_{j}(j=1, \ldots, N)$ in $\mathbb{R}^{n}$ such that $\forall j=1, \ldots, N$, $\forall(x, y) \in \Omega_{j} \times \Omega_{j}$ with $|x-y|<\varepsilon_{0}$, and $\forall t \in \mathbb{R}$ with $M|x-y| \leq t \leq t_{0}$, the line segments $\left[x, x+t h_{j}\right],\left[y, y+t h_{j}\right]$, and $\left[x+t h_{j}, y+t h_{j}\right]$ are all contained in $\Omega$. 
Intuitively, $\Omega$ satisfies the " $\left(C^{\prime}\right)$ condition" if its boundary $\Gamma$ is not "too long". Next we recall two examples from [Mt2, pp. 15-16] (see also [Mt3, pp. 154-156]).

Example 2.1 (Segment and cone conditions). $\Omega$ satisfies the " $\left(\mathrm{C}^{\prime}\right)$ condition" if it obeys the "segment condition". (I.e., if there exists an open cover $\left\{\Omega_{j}\right\}_{j=1}^{N}$ of $\partial \Omega=\Gamma$ and nonzero vectors $h_{j}(j=1, \ldots, N)$ in $\mathbb{R}^{n}$ such that for all $x \in \Omega_{j} \cap \Omega$, we have $\left[x, x+h_{j}\right] \subset \Omega$; see [Ag, p. 11].) This is the case, in particular, if $\Omega$ is Lipschitz [Mt3, p. 155] or if it satisfies a "restricted cone condition" (in the sense of $[\mathrm{Ag}, \mathrm{p} .11]$ ); in the latter case, it follows from [Fe, Theorem 3.2 .39 , p. 275 or Mt2, p. 16], respectively, that $D(=H)=n-1$ and $\mathscr{M}_{D}(\Gamma)<+\infty$.

Example 2.2 (Open sets with cusp). The " $\left(\mathrm{C}^{\prime}\right)$ condition" is also satisfied by open sets of the following form: $\Omega=\left\{x=\left(x_{0}, x^{\prime}\right) \in(0, \delta) \times \mathbb{R}^{n-1}:\left|x^{\prime}\right|<\right.$ $\left.g\left(x_{0}\right)\right\}$, where $n \geq 2$ and $g$ is a continuous nondecreasing function on $(0, \delta)$. Observe that $g$ is allowed to be flat near 0 and hence that $\Omega$ may be pinched at the origin. (One may take, for instance, $g(x)=\exp \left(-x^{-2}\right)$.) Furthermore, note that $g$ may be the Cantor singular function, for instance. (See e.g., [Cn, pp. 55-56 or Md1, pp. 82-83].) Consequently, even though the graph of $g$ is rectifiable and $\Gamma$ may not be "fractal" in the standard sense, the boundary $\Gamma$ can be quite irregular in this case.

We conclude this subsection by providing some general references: for the theory of "fractals", we mention [Ce, Fa, PeRi, Md1, 2], and for its applications to physics, we point out [Md1, 2, PiTo, SMR]. For the theory of elliptic boundary value problems, the reader may wish to consult [Ag, LiMa, Wb], and for the study of spectral asymptotics of elliptic operators, we refer to [CoHi, EdEv, Ho2, ReSi]. An interesting review of Weyl's problem (up to 1976) from a physicist's perspective is provided in [BaHi]. Finally, the basic facts about Sobolev spaces used in this paper can be found in [Ad, Ag].

2.2. Hypotheses and statements of the results. We can now state our hypotheses and present our problem in a precise form:

(2.9) Let $V$ be a closed subspace of $H^{m}(\Omega)$ containing $H_{0}^{m}(\Omega)$ :

$$
H_{0}^{m}(\Omega) \subset V \subset H^{m}(\Omega) .
$$

(2.10) Let $\mathscr{A}$ be a positive, uniformly elliptic and formally selfadjoint operator of order $2 m(m \geq 1)$ defined on $\Omega$ :

$$
\mathscr{A}=\sum_{\substack{|\alpha| \leq m \\|\beta| \leq m}}(-1)^{|\alpha|} D^{\alpha}\left(a_{\alpha \beta}(x) D^{\beta}\right) .
$$

We suppose that $a_{\alpha \beta}=\overline{a_{\alpha \beta}} \in L^{\infty}(\Omega)$ for $|\alpha|,|\beta| \leq m, a_{0} \geq 0$, and that the 
associated bounded hermitian form

$$
a(u, v)=\int_{\Omega} \sum_{\substack{|\alpha| \leq m \\|\beta| \leq m}} a_{\alpha \beta}(x) D^{\alpha} u \overline{D^{\beta} v}, \quad u, v \in V
$$

is coercive on $V$ (in the sense of [Ag or LiMa]); i.e., there exists a positive constant $c_{1}$ such that $c_{1}\|u\|_{H^{m}(\Omega)}^{2} \leq a(u, u), \forall u \in V$.

(2.11) We assume that $\mathscr{A}$ has locally constant leading coefficients on $\Omega$; i.e., for $|\alpha|=|\beta|=m, a_{\alpha \beta}$ is constant on each connected component of $\Omega$.

We consider the following eigenvalue problem:

$$
\mathscr{A} u=\lambda u, \quad u \in V \text {. }
$$

We stress that $\left(\mathbf{P}_{\mathscr{A}}\right)$ is understood in the variational sense; hence, by definition the scalar $\lambda$ is said to be an eigenvalue of $\left(\mathbf{P}_{\mathscr{A}}\right)$ [or is in the "spectrum" of $\left.\left(\mathrm{P}_{\mathscr{A}}\right)\right]$ if there exists a nonzero $u \in V$ such that, with $a$ defined as in (2.10),

$$
a(u, v)=\lambda(u, v)_{L^{2}(\Omega)} \quad \forall v \in V .
$$

When $V=H_{0}^{m}(\Omega)$ [resp., $V=H^{m}(\Omega)$ ], $\left(\mathrm{P}_{\mathscr{A}}\right)$ provides a variational interpretation of the eigenvalue problem: $\mathscr{A} u=\lambda u$ in $\Omega$, with (linear homogeneous) Dirichlet (resp., Neumann) boundary conditions; in this case, we refer to $\left(\mathrm{P}_{\mathscr{A}}\right)$ as the Dirichlet (resp., Neumann) problem. [For example, when $m=1$-as is the case of the usual Laplace operator studied in the introduction and in Theorem 2.3 below-we have, in the variational sense, $u=0$ (resp., $\partial u / \partial n=0)$ on $\Gamma$, where $\partial / \partial n$ denotes a "normal derivative" along $\Gamma$.] Similarly, when $H_{0}^{m}(\Omega) \subset V \subset H^{m}(\Omega),\left(\mathrm{P}_{\mathscr{A}}\right)$ enables us to treat (linear homogeneous) mixed boundary conditions on $\Gamma$. We then refer to $\left(\mathrm{P}_{\mathscr{A}}\right)$ as the mixed (Dirichlet-Neumann) problem. (See [Ag, Chapter 10 or LiMa, Chapter 9].) We note that it would be more accurate to refer to the general case when $H_{0}^{m}(\Omega) \subset V \subset H^{m}(\Omega)$ as defining "abstract boundary conditions", which includes, in particular, the case of mixed boundary conditions. (See, e.g., [Ag, p. 142].) However, we shall continue throughout the text using instead the more suggestive expression "mixed Dirichlet-Neumann boundary conditions".

(2.13) We assume that $\Omega$ satisfies the " $\left(C^{\prime}\right)$ condition" only for the Neumann or, more generally, the mixed Dirichlet-Neumann problem [i.e., when $V \neq$ $\left.H_{0}^{m}(\Omega)\right]$. (See Definition 2.2 above.)

We point out that for the Dirichlet problem, $\Omega$ is assumed to be an arbitrary (nonempty) bounded open set in $\mathbb{R}^{n}$.

Since $\Omega$ is bounded, it is known that the spectrum of $\left(\mathrm{P}_{\mathscr{A}}\right)$ is discrete and consists of an infinite sequence $\left\{\lambda_{i}\right\}_{i=1}^{\infty}$ of positive eigenvalues of finite multiplicity, written in increasing order (according to multiplicity) as follows:

$$
0<\lambda_{1} \leq \lambda_{2} \leq \cdots \leq \lambda_{i} \leq \cdots \text {, with } \lambda_{i} \rightarrow+\infty \text { as } i \rightarrow \infty .
$$

Moreover, these eigenvalues are given by the "max-min formula" (see, e.g., [Mt3 or FlLa 1, p. 314, in conjunction with 4.B, pp. 315-316]):

$$
\frac{1}{\lambda_{i}}=\max _{F_{i} \in \mathscr{S}_{i}} \min _{u \in F_{i}}\left\{\int_{\Omega}|u|^{2}: a(u, u) \leq 1\right\},
$$


where, for $i \geq 1, \mathscr{F}_{i}$ is the set of $i$-dimensional subspaces of $V$.

Let $N(\lambda)$ denote the "counting function" for the variational problem $\left(\mathrm{P}_{\mathscr{A}}\right)$; that is, for $\lambda>0, N(\lambda)$ is the number of positive eigenvalues (counting multiplicity) which do not exceed $\lambda$ :

$$
N(\lambda)=\#\left\{i \geq 1: \lambda_{i} \leq \lambda\right\}=\sum_{\lambda_{i} \leq \lambda} 1 .
$$

The leading asymptotics of $N(\lambda)$ are provided by the analogue of Hermann Weyl's formula in the present more general situation (see, e.g., [Mt1, 2; Mt3, Theorems 5.1 and 5.2, p. 175]):

$$
N(\lambda) \sim \mu_{\mathscr{A}}^{\prime}(\Omega) \lambda^{n / 2 m} \text { as } \lambda \rightarrow+\infty .
$$

[Recall that (2.17) means that $N(\lambda)=\mu_{\mathscr{A}}^{\prime}(\Omega) \lambda^{n / 2 m}+o\left(\lambda^{n / 2 m}\right)$, as $\lambda \rightarrow+\infty$. ] Here, $\mu_{\mathscr{A}}^{\prime}(\Omega)$, the "Browder-Gårding measure" of $\Omega$, is a constant depending only on $n, \Omega$, and the leading coefficients of $\mathscr{A}$ :

$$
\mu_{\mathscr{A}}^{\prime}(\Omega)=\int_{\Omega} \mu_{\mathscr{A}}^{\prime}(x) d x
$$

where the "Browder-Gårding density" is defined by

$$
\mu_{\mathscr{A}}^{\prime}(x)=(2 \pi)^{-n}\left|\left\{\xi \in \mathbb{R}^{n}: a^{\prime}(x, \xi)<1\right\}\right|_{n},
$$

with $a^{\prime}(x, \xi)$-the leading symbol of the quadratic form $a$ associated with $\mathscr{A}$ - given by

$$
a^{\prime}(x, \xi)=\sum_{|\alpha|=|\beta|=m} a_{\alpha \beta}(x) \xi^{\alpha+\beta}, \quad(x, \xi) \in \Omega \times \mathbb{R}^{n} .
$$

Remarks 2.2. (a) Of course, if $\mathscr{A}$ is assumed to have constant leading coefficients, then $\mu_{\mathscr{A}}^{\prime}(\Omega)=\tilde{\mu}_{\mathscr{A}}^{\prime}|\Omega|_{n}$, where $\tilde{\mu}_{\mathscr{A}}^{\prime}\left(\equiv \mu_{\mathscr{A}}^{\prime}(x)\right.$ in this case $)$ is a constant depending only on the leading part of $\mathscr{A}$. [For example, if $\mathscr{A}=-\Delta+1$, then $\tilde{\mu}_{\mathscr{A}}^{\prime}=(2 \pi)^{-n} \mathscr{B}_{n}$.]

(b) Recall that (for the Laplacian and) for the Neumann problem, if the " $\left(C^{\prime}\right)$ condition" does not hold, then-according to [Mt3, §VII.1, pp. 200-204]-even the leading asymptotics of $N(\lambda)$ need not be given by Weyl's formula (2.17)and hence our remainder estimate given in Corollary 2.2 (and Theorem 2.3) below could not possibly hold in this case. (See also [Mt1, FlMt].) This is so even if the Neumann spectrum is assumed to be discrete, which is the case, of course, if the " $\left(C^{\prime}\right)$ condition" is satisfied. (Naturally, the spectrum of the Dirichlet problem is always discrete because $\Omega$ is bounded; see, e.g., [EdEv, $\S 4.9$, pp. 272-274, and Theorem 3.6, p. 227] as well as [M, $\S \S 4.10-4.11$, pp. 249-268].)

We next state our main results, in which we obtain a remainder estimate associated with (2.17) and expressed in terms of the "fractal dimension" of the boundary $\Gamma$ : the Minkowski dimension $\widetilde{D}$ or $D$, as in (2.7) or (2.4), respectively, according to whether we consider Dirichlet or Neumann boundary 
conditions. This remainder estimate is provided in Corollaries 2.1 and 2.2 below and will be deduced from the following theorem which-together with many of the results of this paper-is announced in [La9].

Theorem 2.1. Assume that the above hypotheses are satisfied. Let $d \in[n-1, n]$ be such that $\widetilde{\mathscr{M}}_{d}(\Gamma)<+\infty$ (resp., $\left.\mathscr{M}_{d}(\Gamma)<+\infty\right)$ in the case of Dirichlet (resp., mixed Dirichlet-Neumann) boundary conditions. Then the following remainder estimate holds:

(i) If $d \in(n-1, n]$, then

$$
N(\lambda)=\mu_{\mathscr{A}}^{\prime}(\Omega) \lambda^{n / 2 m}+O\left(\lambda^{d / 2 m}\right) \text { as } \lambda \rightarrow+\infty .
$$

(ii) If $d=n-1$, then

$$
N(\lambda)=\mu_{\mathscr{A}}^{\prime}(\Omega) \lambda^{n / 2 m}+O\left(\lambda^{d / 2 m} \log \lambda\right) \text { as } \lambda \rightarrow+\infty .
$$

Here, the constant $\mu_{\mathscr{A}}^{\prime}(\Omega)$ is defined by (2.18); further, $\mathscr{M}_{d}=\mathscr{M}_{d}(\Gamma)$ and $\widetilde{\mathscr{M}_{d}}=\widetilde{\mathscr{M}}_{d}(\Gamma)$ are given by (2.3) and (2.6), respectively.

Remarks 2.3. (a) Since, clearly, $\widetilde{\mathscr{M}}_{d} \leq \mathscr{M}_{d}, \widetilde{\mathscr{M}}_{d}$ is finite whenever $\mathscr{M}_{d}$ is. Moreover, since for the same reason, $D \leq \widetilde{D}$-as was noted in Remark 2.1(a)all the results stated for the Dirichlet problem in Corollary 2.1 and Theorems 2.2-2.3 below still hold a fortiori if $D$ and $\widetilde{\mathscr{M}}_{\widetilde{D}}$ are replaced by $D$ and $\mathscr{M}_{D}$, respectively. (This justifies, in particular, the way we stated our results in the introduction; see Remark 1.2.)

(b) Case (ii) of Theorem 2.1, in which $d=n-1$, and with the additional assumption that $\widetilde{\mathscr{M}}_{n-1}$ [resp., $\left.\mathscr{M}_{n-1}(\Gamma)\right]$ is finite, corresponds to a "nonfractal" (but possibly "nonsmooth") boundary $\Gamma$ and was already obtained in [Mt3, Theorem 6.1, p. 195]. (See also [Mt2, Theorem, p. 16].) Note that in [Mt2,3], Métivier was not making explicit use of the notion of Minkowski dimension. Further, observe that since $D \geq H \geq n-1$, the condition $d=n-1$ with $\mathscr{M}_{d}<+\infty$ implies that $D=H=n-1$, the topological dimension of $\Gamma$; here, $D=D(\Gamma)$ [resp., $H=H(\Gamma)$ ] denotes the Minkowski [resp., Hausdorff] dimension of $\Gamma$.

(c) In our proof of Theorem 2.1, we shall be able to treat cases (i) and (ii) in parallel. However, the derivation of case (i), where $\Gamma$ may be "fractal", will be the most delicate one.

Our main results are now direct consequences of Theorem 2.1. For the sake of clarity, we shall state them separately for Dirichlet and for mixed DirichletNeumann boundary conditions.

Corollary 2.1 (Dirichlet boundary conditions). Let $\Omega$ be an arbitrary (nonempty) bounded open set in $\mathbb{R}^{n}$, with boundary $\Gamma$, and let $\mathscr{A}$ be a positive elliptic operator of order $2 m$ on $\Omega$ satisfying hypotheses (2.9) through (2.11). Consider the variational Dirichlet problem $\left(\mathrm{P}_{\mathscr{A}}\right)$ [i.e., $V=H_{0}^{m}(\Omega)$ ]. Let $\widetilde{D}=\widetilde{D}(\Gamma) \epsilon$ $[n-1, n]$ be the Minkowski dimension of $\Gamma$, relative to $\Omega$. Then we have the 
following remainder estimates:

(i) If $\widetilde{D} \in(n-1, n]$ (i.e., if $\Gamma$ is "fractal"), then estimate (2.19) holds for all $d>\widetilde{D}$.

(ii) If $\widetilde{D}=n-1$, then estimate (2.20) holds for all $d>\widetilde{D}$.

Furthermore, except possibly in the degenerate case when $\widetilde{\mathscr{M}}_{\widetilde{D}}(\Gamma)$ is infinite, estimate (2.19) [resp., (2.20)] also holds with $d=\widetilde{D}$ in case (i) [resp., (ii)].

Here, $\widetilde{\mathscr{M}}_{\widetilde{D}}(\Gamma)$, as defined in (2.6), denotes the $\widetilde{D}$-dimensional upper Minkowski content of $\Gamma$, relative to $\Omega$.

Note that Corollary 2.1 follows from Theorem 2.1 since according to Definition $2.1, d>\widetilde{D}$ implies that $\widetilde{\mathscr{M}}_{d}<+\infty$. Since, similarly, $\mathscr{M}_{d}$ is finite for $d>D$, Theorem 2.1 also yields the next corollary, which applies in particular to Neumann boundary conditions [i.e., $V=H^{m}(\Omega)$ ].

Corollary 2.2 (Mixed Dirichlet-Neumann boundary conditions). Let $\Omega, \mathscr{A}$, and $V$ satisfy hypotheses (2.9) through (2.11) and (2.13). Recall in particular that the bounded open set $\Omega$ must obey the " $\left(\mathrm{C}^{\prime}\right)$ condition". Consider the variational mixed Dirichlet-Neumann problem $\left(\mathrm{P}_{\mathscr{A}}\right)$ [i.e., $\left.H_{0}^{m}(\Omega) \subset V \subset H^{m}(\Omega)\right]$. Let $D=D(\Gamma) \in[n-1, n]$ be the Minkowski dimension of the boundary $\Gamma$. Then we have the following remainder estimates:

(i) If $D \in(n-1, n]$ (i.e., if $\Gamma$ is "fractal"), then estimate (2.19) holds for all $d>D$.

(ii) If $D=n-1$, then estimate (2.20) holds for all $d>D$.

Furthermore, except possibly in the degenerate case when $\mathscr{M}_{D}(\Gamma)$ is infinite, estimate (2.19) [resp., (2.20)] also holds with $d=D$ in case (i) [resp., (ii)].

Here, $\mathscr{M}_{D}=\mathscr{M}_{D}(\Gamma)$, as defined in (2.3), denotes the D-dimensional upper Minkowski content of $\Gamma$.

Remarks 2.4. (a) In some sense, $\widetilde{D}$ (resp., $D$ ) is related to the "intrinsic" (resp., "extrinsic") geometry of $\bar{\Omega}$. Hence, from the point of view of partial differential equations, it is natural to use $\widetilde{D}$ (resp., $D$ ) to study Dirichlet (resp., Neumann) boundary conditions.

(b) Assume that $\widetilde{\mathscr{M}}_{\widetilde{D}}$ (resp., $\left.\mathscr{M}_{D}\right)=+\infty$. Then, clearly, in the conclusion of Corollary 2.1(i) [resp., 2.2(i)], we can substitute $o\left(\lambda^{d / 2 m}\right)$ for $O\left(\lambda^{d / 2 m}\right)$ if $d>\widetilde{D}($ resp., $>D)$. [This follows since $O\left(\lambda^{d / 2 m}\right)=o\left(\lambda^{d^{\prime} / 2 m}\right), \forall d^{\prime}>d$.] Further, in the conclusion of Corollary 2.1(ii) [resp., 2.2(ii)], we can substitute $o\left(\lambda^{d / 2 m}\right)$ for $O\left(\lambda^{d / 2 m} \log \lambda\right)$ if $d>n-1=\widetilde{D}$ (resp., $\left.=D\right)$. [To see this, applyfor a fixed $d>n-1$-Corollary 2.1(ii) [resp., 2.2(ii)] to $d^{\prime} \in(n-1, d)$ and note that $O\left(\lambda^{d^{\prime} / 2 m} \log \lambda\right)=o\left(\lambda^{d / 2 m}\right)$.] Finally, we point out that since $\widetilde{D} \leq D \leq n$, we are really only interested in those $d$ 's such that $d \leq n$. [A similar remark applies to the remaining results of this section.]

(c) The latter part of Corollary 2.1 (resp., 2.2) when $\widetilde{\mathscr{M}}_{\widetilde{D}}\left(\right.$ resp., $\left.\mathscr{M}_{D}\right)<+\infty$, often applies in practice. [See Remark 2.1(b).] 
(d) In the most "fractal" case when $\widetilde{D}=n$ (resp., $D=n$ ), the conclusion of Corollary 2.1 (resp., 2.2) is less informative than the (generalized) H. Weyl's formula (2.17). Further, we know that in this case, the asymptotics of $N(\lambda)$ need not have a second term of the conjectured form. (See [ $\mathrm{BrCa}$, Example 1, p. 106] and Remark 5.5(b) below.) It would be interesting to find out what precisely happens in this situation.

(e) By using more general definitions of $D$ and $\widetilde{D}$, based on a broader class of functions than the power functions or on a metric other than the Euclidean one, we could refine some of the results obtained in this paper.

(f) In all our results concerning the Neumann problem, it would suffice to assume that the bounded open set $\Omega$ is locally diffeomorphic (in the sense of [Mt3, p. 156]) to one that satisfies the " $\left(\mathrm{C}^{\prime}\right)$ condition". Intuitively, such a relaxed condition allows $\Omega$ not to remain on just "one side of its boundary" $\Gamma$, in which case we may have $\widetilde{D} \neq D$ (i.e., $\widetilde{D}<D$ ). [This generalization and that mentioned in the next comment hold true because Proposition $4.5^{\prime}$ (and hence the counterpart of Proposition 4.6) extends to these situations.]

Some further results about the Neumann problem are given at the end of §4.2. (See Theorem 4.1.) We shall show, in particular, that the " $\left(C^{\prime}\right)$ condition" can be replaced by the "extension property" (i.e., the existence of a continuous linear extension map from $H^{m}(\Omega)$ to $H^{m}\left(\mathbb{R}^{n}\right)$ ). (See Definition 4.3.) This hypothesis is satisfied, for example, by domains bounded by "quasicircles" (i.e., the image of circles under quasiconformal mappings). (See [VGL, Jn; M, pp. 70-71].) We point out that in the latter case, the boundary $\Gamma$ can be extremely irregular [GeVa]. This generalization is noteworthy in view of the interest of "quasidisks" (and their higher-dimensional analogues) in harmonic analysis.

Next, we consider the "partition function" $Z(t)$ associated with $\left(\mathrm{P}_{\mathscr{A}}\right)$ :

$$
Z(t):=\int_{0}^{\infty} e^{-\lambda t} d N(\lambda)=\sum_{i=1}^{\infty} e^{-\lambda_{i} t}
$$

recall that $Z(t)$ is nothing but the trace of the heat semigroup: $Z(t)=\operatorname{Tr}\left(e^{-t \mathscr{8}}\right)$. (See, e.g., [Si] and, for smooth domains, [Gi].) By a simple Abelian argument (e.g., a refinement of $[\mathrm{Si}$, Theorem 10.2, p. 107] given in Theorem A of Appendix A), we deduce the following result from the analogue of Corollaries 2.1 and 2.2 .

Theorem 2.2. Assume that hypotheses (2.9) through (2.11) and-only for the mixed problem-(2.13), are satisfied. Then, for the Dirichlet (resp., mixed Dirichlet-Neumann) problem $\left(\mathrm{P}_{\mathscr{A}}\right)$, the following remainder estimate holds for all $d>\widetilde{D}($ resp., all $d>D)$ :

$$
Z(t)=\mu_{\mathscr{A}}^{\prime}(\Omega)\left(\frac{n}{2 m}\right) ! t^{-n / 2 m}+O\left(t^{-d / 2 m}\right) \text { as } t \rightarrow 0^{+},
$$

where $\mu_{\mathscr{A}}^{\prime}(\Omega)$ is given by (2.18). Furthermore, if $D \in(n-1, n]$ and $\widetilde{\mathscr{M}}_{\widetilde{D}}(\Gamma)<$ $+\infty$ (resp., $D \in(n-1, n]$ and $\left.\mathscr{M}_{D}(\Gamma)<+\infty\right)$, then (2.22) still holds with $d=\widetilde{D}($ resp., $d=D)$. 
Moreover, the present theorem applies without change to $\mathscr{A}:=(-\Delta)^{m}$, with $m \geq 1 ;$ in this case, $\mu_{\mathscr{A}}^{\prime}(\Omega)(n / 2 m) !=(4 \pi)^{-n / 2}|\Omega|_{n}(n / 2 m) ! /(n / 2) !$.

Theorem 2.2 follows from (but by Remark 2.5(a) below does not necessarily imply) Theorem 2.1 (as well as Corollaries $2.1-2.2$ ) by application of the aforementioned Abelian argument. Theorem 2.1 will be established in $\S 4$ while Theorem 2.2 will be proved in Appendix A.

Remarks 2.5. (a) Beyond the leading term (for which a Tauberian theorem due to Karamata can be used [Ka, Si]), it is not known how to obtain precise information about the asymptotics of $N(\lambda)$ from that of $Z(t)$. (See, e.g., [Ya, p. 114].)

(b) We stress that for the Dirichlet problem, Theorem 2.1 does not require any regularity about the boundary $\Gamma$. Some further results about the asymptotics of the partition function $Z(t)$ are given in [ $\mathrm{BrCa}, \S 3]$ under certain regularity assumptions. [When comparing the constants involved, the reader should be aware of the fact that some authors (e.g., [Ka, Si, and $\mathrm{BrCa}]$ ) use the probabilistic (negative) Laplacian $-(1 / 2) \Delta$ instead of $-\Delta$; hence, with our notation, their partition function is equal to $Z(t / 2)$.]

(c) The (pre-Tauberian) results about $Z(t)$ obtained in [BrCa, $\$ 3]$ - which are based on probabilistic methods-are stated in the case of the Dirichlet Laplacian and include Theorem 2.2 in this special case. On the other hand, our results are valid for positive elliptic operators of any order (as well as Neumann boundary conditions), as is seen from Theorem 2.2.

We now consider the case of the Laplace operator $\Delta=\sum_{k=1}^{n} \partial^{2} / \partial x_{k}^{2}$. Hence we assume that $\mathscr{A}=-\Delta, m=1$, and simply write $(\mathrm{P})$ instead of $\left(\mathrm{P}_{\mathscr{A}}\right):-\Delta u=$ $\lambda u, u \in V$, where $H_{0}^{1}(\Omega) \subset V \subset H^{1}(\Omega)$, as in (2.9); further, (P) is understood in the variational sense and $a(u, v):=\int_{\Omega} \nabla u \nabla \bar{v}$. The only difference with the previous case is that $-\Delta$ is nonnegative instead of being (strictly) positive. [Recall that the Dirichlet problem $(\mathrm{P})$ has only positive eigenvalues whereas 0 is an eigenvalue of the Neumann problem $(\mathrm{P})$; moreover, $N(\lambda)$ still denotes the number of (strictly) positive eigenvalues of $(\mathbf{P})$ not exceeding $\lambda$.] We thus obtain Theorem 2.3 below by applying Theorem 2.1 (and its corollaries) to the (strictly) positive operator $\mathscr{A}_{\tau}:=-\Delta+\tau$, with $\tau$ a positive constant, and then by letting $\tau \rightarrow 0^{+}$; note that the implicit constants involved in the remainder term can be chosen to be independent of $\tau$ since $-\Delta$, the leading part of $\mathscr{A}_{\tau}$, does not depend on $\tau$.

We now briefly summarize our results for the Laplacian:

Theorem 2.3. Assume that $\mathscr{A}:=-\Delta$ and that hypothesis (2.9) is satisfied with $m=1$. Further, only for mixed Dirichlet-Neumann boundary conditions, we also suppose that (2.13) holds. For the Dirichlet problem, $\Omega$ is allowed to be an arbitrary (nonempty) bounded subset of $\mathbb{R}^{n}$. Let $\mathscr{B}_{n}=\pi^{n / 2} /(n / 2)$ ! denote the volume of the unit ball in $\mathbb{R}^{n}$. 
Then, Theorems 2.1-2.2, as well as Corollaries 2.1-2.2, apply without change in this case. In particular, estimate (2.19) reads as follows:

$$
N(\lambda)=(2 \pi)^{-n} \mathscr{B}_{n}|\Omega|_{n} \lambda^{n / 2}+O\left(\lambda^{d / 2}\right) \text { as } \lambda \rightarrow+\infty ;
$$

moreover, estimate (2.22) becomes

$$
Z(t)=(4 \pi)^{-n / 2}|\Omega|_{n} t^{-n / 2}+O\left(t^{-d / 2}\right) \text { as } t \rightarrow 0^{+} .
$$

Remarks 2.6. (a) Since $\widetilde{D} \leq D$, we recover the results concerning the Laplacian stated in the introduction [Theorem 1.1 as well as estimates (1.8) and (1.10)].

(b) The special case of estimate (2.23) for the Dirichlet Laplacian (with $D$ instead of $\widetilde{D}$ ), is announced in [LaFl].

(c) We could of course state the counterpart of Theorem 2.3 for the iterated Laplacian $\mathscr{A}:=(-\Delta)^{m}$, with $m \geq 1$.

(d) Our results are in some sense "best possible". Indeed, [BrCa, Example 2] provides an instance where $0<\widetilde{\mathscr{M}}_{\widetilde{D}}<+\infty$ and for the Dirichlet Laplacian, with $n=2$, we have $\left.\left|N(\lambda)-(2 \pi)^{-n} \mathscr{B}_{n}\right| \Omega\right|_{n} \lambda^{n / 2} \mid \approx \lambda^{\widetilde{D} / 2}$, as $\lambda \rightarrow+\infty$; hence our remainder estimate $(2.23)$ cannot be improved in this case. (See Example 5.2 below.) We exhibit an even simpler example of this kind (with $n=1$ ) in Example 5.1. Actually, for all $n \geq 1$, Examples 5.1 and $5.1^{\prime}$ yield a oneparameter family of examples for which our remainder estimate is sharp and the Minkowski dimension $D$ (equal to $\widetilde{D}$ in this case) takes on every value in $(n-1, n)$. Further, Berry's original conjecture-expressed in terms of the Hausdorff instead of the Minkowski dimension-obviously fails for any of these examples.

(e) In this paper, we consider several facets of the problem of "fractality" in space. On the other hand, certain aspects of our recent work on the "FeynmanKac formula with a Lebesgue-Stieltjes measure" [La4-7] (see also [JhLa]) are related to the problem of "fractality" in time.

\section{Minkowski AND HAUSdORfF Dimensions}

Generally, the Hausdorff dimension has been the preferred measure of "fractality" or irregularity; there are both historical and theoretical reasons for this. However, many practitioners-often without being fully aware of it-have been using the Minkowski dimension instead, in one disguised form or another. In this section, we shall briefly expand upon these remarks and review and compare some of the main properties of the Minkowski and Hausdorff dimensions.

Many of the results of this section-that pertain among other fields to the area of geometric measure theory-are probably known to a few experts but are not easily accessible to most readers since they are scattered throughout the literature.

For simplicity, we shall work in $\mathbb{R}^{n}(n \geq 1)$, equipped with its Euclidean metric. Let $A$ be a subset of $\mathbb{R}^{n}$.

Definition 3.1. Given $d \geq 0$, we set $\mathscr{H}_{d}(A)=\lim _{\varepsilon \rightarrow 0^{+}}\left\{\inf \sum_{i=1}^{\infty}\left(r_{i}\right)^{d}\right\}$, where the infimum is taken over all (countable) coverings of $A$ by open balls $\left\{B_{i}\right\}_{i=1}^{\infty}$ 
of radius $r_{i} \leq \varepsilon$. The number $\mathscr{H}_{d}(A)$ in $[0,+\infty]$ is called the $d$-dimensional Hausdorff (outer) measure of $A$ and $H(A):=\inf \left\{d \geq 0: \mathscr{H}_{d}(A)=0\right\}=$ $\sup \left\{d \geq 0: \mathscr{H}_{d}(A)=+\infty\right\}$ is called the Hausdorff dimension of $A$.

When $d=H(A)$, we have $\mathscr{H}_{d}(A) \in[0,+\infty]$. For detailed information about the Hausdorff dimension, we refer to [Ro or Fa].

In Definition 3.1, the $B_{i}$ 's can also be assumed to be cubes of side $r_{i} \leq \varepsilon$ (or even arbitrary subsets of $\mathbb{R}^{n}$ of diameter $r_{i} \leq \varepsilon$ ). In any case, the $r_{i}$ 's are allowed to vary between 0 and $\varepsilon$. This is in contrast with the (alternative) definition of the Minkowski dimension provided by Corollary 3.1 below, where the radius of the balls (or the sides of the squares) must be exactly equal to $\varepsilon$.

The next results (Proposition 3.1 and its corollaries) illustrate this basic difference between the Minkowski and Hausdorff dimensions. In addition, they have led us to reformulate our initial remainder estimate in terms of the Minkowski dimension and-as will be explained towards the end of this section-to establish connections with our earlier (joint) work [FlLa2] on eigenvalue problems with indefinite weights. (See especially Corollaries 3.1 and 3.3.) Their proof should be useful in following that of our main results in the next section.

Given $\varepsilon>0$, let $\left\{Q_{\zeta}\right\}_{\zeta \in \mathbb{Z}^{n}}$ be a "tessellation" of $\mathbb{R}^{n}$ by a countable family of disjoint open ( $n$-dimensional) cubes of side $\varepsilon$ and center $x_{\zeta}$ such that $\bigcup_{\zeta \in \mathbb{Z}^{n}} \overline{Q_{\zeta}}=\mathbb{R}^{n}$. [In the following, we write $\|$ instead of $\|\left.\right|_{n}$.] If $A \subset \mathbb{R}^{n}$ is bounded, we set

$$
K=K(\varepsilon)=K(\varepsilon ; A):=\left\{\zeta \in \mathbb{Z}^{n}: \overline{Q_{\zeta}} \cap A \neq \varnothing\right\} .
$$

Proposition 3.1. There exist positive constants $c_{1}$ and $c_{2}$-depending only on $n$-such that for all $\varepsilon>0$ and all bounded subsets $A$ of $\mathbb{R}^{n}$, we have, with $A_{\varepsilon}$ as in (2.1):

$$
(\# K(\varepsilon)) \varepsilon^{n} \leq\left|A_{c_{1} \varepsilon}\right| \text { and }\left|A_{\varepsilon}\right| \leq c_{2}(\# K(\varepsilon)) \varepsilon^{n} .
$$

Proof. For notational simplicity, we write $K$ instead of $K(\varepsilon)$. We first establish the first inequality in (3.2). Clearly, by (3.1), $\bigcup_{\zeta \in K} Q_{\zeta} \subset A_{(1+\sqrt{n}) \varepsilon}$; hence, since the cubes $Q_{\zeta}$ are disjoint, we have $\left|\bigcup_{\zeta \in K} Q_{\zeta}\right|=(\# K)\left|Q_{\zeta}\right|=(\# K) \varepsilon^{n} \leq$ $\left|A_{(1+\sqrt{n}) \varepsilon}\right|$. The result follows with $c_{1}=1+\sqrt{n}$ (or, more precisely, any $c_{1}>$ $\sqrt{n})$.

Next we prove the second inequality in (3.2). Observe that there exists $\rho>$ 1 -depending only on $n$-such that $A_{\varepsilon} \subset \bigcup_{\zeta \in K} \overline{\rho Q_{\zeta}}$. [Here, " $\rho Q_{\zeta}$ " denotes the cube of center $x_{\zeta}$ and side $\rho \varepsilon$; we can choose, for example, $\rho=3 \sqrt{n}$.] Note that, by construction, the cubes $\left\{\rho Q_{\zeta}\right\}_{\zeta \in K}$ are not (pairwise) disjoint. We deduce that

$$
\left|A_{\varepsilon}\right| \leq\left|\bigcup_{\zeta \in K} \overline{\rho Q_{\zeta}}\right| \leq \sum_{\zeta \in K}\left|\overline{\rho Q_{\zeta}}\right|=(\# K) \rho^{n} \varepsilon^{n} .
$$

The desired result follows with $c_{2}=(3 \sqrt{n})^{n}$. 
Remarks 3.1. (a) For simplicity, we do not consider here the notion of relative Minkowski dimension introduced in $\S 2.1$; hence, $D(A)$ is given as in Definition 2.1(a) [and Remark 2.1(e)].

(b) As we learned after having obtained this result, a special case of Proposition 3.1 is contained in [Bo].

(c) I wish to thank Dr. Joseph H. G. Fu for a conversation about geometric measure theory.

We now state several consequences of Proposition 3.1:

Corollary 3.1. Let $A$ be a bounded subset of $\mathbb{R}^{n}$. Then

$$
D(A)=\limsup _{\varepsilon \rightarrow 0^{+}} \frac{\log (\# K(\varepsilon))}{\log (1 / \varepsilon)} .
$$

Proof. Let $b$ denote the right-hand side of (3.3). We will deduce from the first inequality in (3.2) that $b \leq D(A)$ and from the second one that $b \geq D(A)$.

Step 1: $b \leq D(A)$. Clearly, it suffices to show that $d>D(A)$ implies $d \geq b$. Hence, fix $d>D(A)$. Since, by Definition 2.1, $\mathscr{M}_{d}(A)=0<+\infty$, there exist positive constants $\varepsilon_{0}$ and $C$ such that $\left|A_{c_{1} \varepsilon}\right| \leq C \varepsilon^{n-d}, \forall \varepsilon<\varepsilon_{0}$. Now, we apply the first inequality in (3.2) and simplify by $\varepsilon^{n}$ to obtain $\# K(\varepsilon) \leq C \varepsilon^{-d}$, $\forall \varepsilon<\varepsilon_{0}$, and conclude that

$$
b:=\limsup _{\varepsilon \rightarrow 0^{+}} \frac{\log (\# K(\varepsilon))}{\log (1 / \varepsilon)} \leq d,
$$

as desired.

Step 2: $b \geq D(A)$. Equivalently, we prove that $d>b$ implies that $d \geq D(A)$. Fix $d>b$. By definition of $b$, there exists $\varepsilon_{0} \in(0,1)$ such that $\forall \varepsilon<\varepsilon_{0}$,

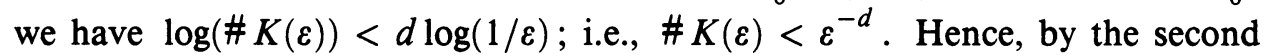
inequality in (3.2), $\varepsilon^{-(n-d)}\left|A_{\varepsilon}\right| \leq c_{2}, \forall \varepsilon<\varepsilon_{0}$, and, by (2.3), $\mathscr{M}_{d}(A) \leq c_{2}<$ $+\infty$. Thus $d \geq D(A)$, as required.

Remarks 3.2. (a) Related characterizations of $D(A)$ can be found in [Tr2] and references therein (particularly [Bo], where the upper limit was omitted). (See, e.g., [Tr2, Corollary 2, p. 61].) Moreover, we note that the notion of Minkowski dimension can be linked with that of Whitney coverings, of frequent use in harmonic analysis (see $[\mathrm{MrVu}])$.

(b) When $n=1$, further equivalent definitions of $D(A)$ are given in [Tr1]; these extend in particular the following useful criterion obtained (in part) in [Ha, Theorem 3.1, p. 707]: let $A \subset \mathbb{R}$ be a compact set; write $[\inf A, \sup A] \backslash A$ as a disjoint union of open intervals $\left\{I_{i}\right\}_{i=1}^{\infty}$ ordered so that their respective lengths $\left\{l_{i}\right\}_{i=1}^{\infty}$ form a nonincreasing sequence. Then $D(A)$ is given by the following "Taylor-Besicovitch index": $D(A)=\inf \left\{\rho \geq 0: \sum_{i=1}^{\infty}\left(l_{i}\right)^{\rho}<+\infty\right\}$.

It follows from Corollary 3.1 (as well as [Tr2, Corollary 2, p. 61]) that the Minkowski dimension (in $\mathbb{R}^{n}$ ) is nothing but the "metric dimension", the "entropy (or information) dimension" (Pontrjagin-Schnirelmann/Gelfand/Hawkes), 
the " $\varepsilon$-capacity dimension", the "logarithmic density", as well as the "box dimension" frequently used by the practitioners of "fractal geometry". (See, for example, [Tr2 and Ce, especially the papers by S. Dubuc, pp. 16-38, and J. Peyrière, pp. 151-157] for precise references on these subjects.)

Since it is defined in a constructive manner, $D(A)$ can be measured "experimentally" (see, e.g., [Ce, p. 152]) and calculated with the help of a computer (see, e.g., [Ce, p. 31]); Corollary 3.1-that connects $D(A)$ with "tessellations" of $\mathbb{R}^{n}$ by cubes of equal size-is the most useful in this context.

The next result (see, e.g., [Ce; $\mathrm{MrVu}$, p. 26; Tr2, p. 60]) can easily be deduced from Corollary 3.1 (and its method of proof).

Proposition 3.2. For every $A \subset \mathbb{R}^{n}$, we have $H(A) \leq D(A)$.

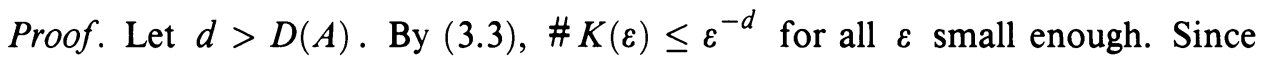
$A \subset \bigcup_{\zeta \in K(\varepsilon)} \overline{Q_{\zeta}}$, it follows from Definition 3.1 that

$$
\mathscr{H}_{d}(A) \leq \lim \sup \left(\# K(\varepsilon)(\sqrt{n} \varepsilon)^{d}\right) \leq n^{d / 2}<+\infty
$$

and hence $H(A) \leq d$. Since $d>D(A)$ is arbitrary, we conclude that $D(A) \leq$ $H(A)$.

This enables us in particular to justify an equality that was often used in $\S 2$. (See Remark 2.1(a).)

Corollary 3.2. If $A:=\partial \omega$, where $\omega$ is a nonempty bounded open subset of $\mathbb{R}^{n}$, then $D(A) \in[n-1, n]$.

Proof. Recall from [HrWa, §VII.4, p. 107] that $H(A) \geq T(A)$, where $T(A)$ denotes the topological dimension of $A$. Moreover, since the open set $\omega$ is neither empty nor dense, we have according to [HrWa, Corollary 2, p. 46]: $T(A)=T(\partial \omega)=T(\omega)-1=n-1$. Since obviously $D(A) \leq n$, the conclusion follows from Proposition 3.2. [We note that the same argument shows that Corollary 3.2 extends to any nonempty open set $\omega$ that is not dense in $\mathbb{R}^{n}$.]

Recall that $\mathscr{H}_{d}(\cdot)$ is an outer measure and induces a ( $\sigma$-additive) measure on (the Borel subsets of) $\mathbb{R}^{n}$. (See, e.g., [Fa, Chapter 1].) It follows from the first property that the Hausdorff dimension satisfies $(*): H\left(\bigcup_{i=1}^{\infty} A_{i}\right)=$ $\sup _{i \geq 1} H\left(A_{i}\right)$. (See, e.g., [Tr2].) In particular, $H(A)=0$ whenever $A \subset \mathbb{R}^{n}$ is countable.

On the other hand, the upper Minkowski content $\mathscr{M}_{d}(\cdot)$ is only a premeasure on $\mathbb{R}^{n}$ and thus $(*)$ fails to hold (except for finite families); this follows since, according to Definition 2.1, $\mathscr{M}_{d}(A)=\mathscr{M}_{d}(\bar{A})$ and hence $D(A)=D(\bar{A})$.

These properties of $D(\cdot)$ are certainly unpleasant from a theoretical point of view; however, as will be explained below and in $\$ 5.1$, and as should be clear from the proof of Theorem 2.1 given in $\S 4.2$, they enable us to extract information-essential in our context-which is otherwise "invisible" to $H(\cdot)$ or to other notions of "fractal" dimension. (See especially Examples 5.1-5.1' and Remarks 5.2(a),(b).) 
We now give several examples of sets $A \subset \mathbb{R}^{n}$ for which $H(A) \neq D(A)$; the first three assume that $n=1$ but similar examples can be obtained in $\mathbb{R}^{n}$ $(n \geq 2)$.

Example 3.1. Let $A$ be the set of rational numbers in $[0,1]$. Then $H(A)=0$ and $D(A)=1$. [In fact, $A$ is countable and $D(A)=D(\bar{A})=D([0,1])=1$.]

Of course, the same conclusion holds if $A$ is any countable dense subset of $[0,1]$.

The following simple example will be useful to illustrate our main results and develop further intuition for the relationship between "fractal" dimension(s) and spectral theory. (See Examples 5.1 and $5.1^{\prime}$.)

Example 3.2. Fix a positive real number $a$. Let $A=\left\{i^{-a}: i=1,2, \ldots\right\}$. Then $H(A)=0$ and $D(A)=(a+1)^{-1}$. [In fact, $H(A)=0$ since $A$ is countable; further,

$$
\begin{aligned}
D(A) & =D(\bar{A})=D(A \cup\{0\})=\inf \left\{\rho: \sum_{i=1}^{\infty}\left(l_{i}\right)^{\rho}<+\infty\right\} \\
& =\inf \left\{\rho: \rho>(a+1)^{-1}\right\}=(a+1)^{-1} .
\end{aligned}
$$

Note that $[0,1] \backslash \bar{A}=\bigcup_{i=1}^{\infty}\left((i+1)^{-a}, i^{-a}\right)$ and hence we can apply the result recalled in Remark 3.2(b) with $l_{i}:=i^{-a}-(i+1)^{-a} \approx i^{-(a+1)}$, as $i \rightarrow \infty$; for a different derivation, we refer to Appendix $\mathrm{C}$ (in conjunction with Remark 5.1).] Observe that as the parameter $a$ varies in $(0,+\infty), D(A)$ takes on every value in $(0,1)$.

Example 3.3. Let $A$ be a perfect symmetric set in $[0,1]: A=\bigcap_{i=0}^{\infty} K_{i}$, where $K_{i}$ is the union of $2^{i}$ disjoint intervals of length $a_{i}$, such that $a_{0}=1$ and $a_{i+1}<a_{i} / 2$. Then

$$
H(A)=\liminf _{i \rightarrow \infty} \frac{\log 2^{i}}{\log \left(1 / a_{i}\right)} \quad \text { and } \quad D(A)=\limsup _{i \rightarrow \infty} \frac{\log 2^{i}}{\log \left(1 / a_{i}\right)} .
$$

(Cf. [KhSa, Chapters I and II; Tr2, Example 1, pp. 66-67].) In general, $H(A)<$ $D(A)$; for the triadic Cantor set, however, $a_{i}=3^{-i}$ and hence $H(A)=D(A)=$ $\log 2 / \log 3$. [The latter fact can also be deduced from Lemma 3.1(i) below; see Remark 3.4(b).]

A higher-dimensional analogue of this example [Tr2, p. 69] shows that for every $n \geq 1$, there exists a "generalized Cantor set" $A \subset \mathbb{R}^{n}$ such that $D(A)-$ $H(A)$ takes any preassigned value in $[0, n]$.

Example 3.4. Let $A \subset \mathbb{R}^{2}$ be the graph of a planar spiral. Then $H(A)=1$, the topological dimension of $A$, whereas $D(A)$ takes every value in $[1,2]$, as $A$ runs through the family of spirals. (Cf. [DMT]; see also Example 5.3 below in which these facts are used to illustrate our results.) Again, in this case, the Minkowski dimension is a more refined measure of "complexity" than the Hausdorff dimension. 
Remarks 3.3. (a) An additional and instructive example is provided by [ $\mathrm{BrCa}$, Example 2]. (See Example 5.2 below; in [BrCa], it is shown that $H<\widetilde{D}$, and whence $H \neq D$, since $\widetilde{D} \leq D$.)

(b) Naturally, if $A$ is sufficiently regular, we have $H(A)=D(A)$. For instance, if $A \subset \mathbb{R}^{n}$ (is closed and) $k$-rectifiable (i.e., is the image under a Lipschitz map of a compact set $B \subset \mathbb{R}^{k}$ ), then $H=D=k$. (See [Fe, Theorem 3.2.39, p. 275].)

Another case when the Minkowski and Hausdorff dimensions coincide is when $A$ is "self-similar". (See, e.g., [Tr3, Lemma 5; MrVu] and Remark 3.4(a) below.)

Lemma 3.1. (i) [Self-similar sets] Let $N$ be an integer $\geq 2$. Assume that $A \subset$ $\mathbb{R}^{n}$ is (strictly) "self-similar"; i.e., $A$ is the union of $N$ disjoint sets $A_{i}$ ( $i=$ $1, \ldots, N)$ each of which is similar to $A$ with ratio $r \in(0,1)$. Then $D(A)=$ $H(A)=\log N / \log (1 / r)$.

(ii) More generally, if the $A_{i}$ 's above are similar to $A$ with possibly different ratios $r_{i} \in(0,1), i=1, \ldots, N$, then $D(A)$ is equal to the "similarity dimension" of $A$; i.e., $D(A)$ is the unique positive real number $d$ such that $\sum_{i=1}^{N}\left(r_{i}\right)^{d}=1$.

Remarks 3.4. (a) More generally, Lemma 3.1(i) extends to "self-similar fractals" that satisfy the "open set condition" (in the sense of [Hu or $\mathrm{Fa}, \S 8.3]$ ); see, e.g., [MrVu, Theorem 4.19, p. 20]. In addition, in this case $\mathscr{M}_{D}(A)<+\infty$; see [MrVu, Remark 4.20, p. 29].

(b) Many classical "fractals" are "self-similar" in the above sense; for instance, if $A$ is the triadic Cantor set [Ch; Mdl, pp. 80-81], we have $N=$ $2, r=1 / 3$, and hence $D(A)=H(A)=\log 2 / \log 3$. Further, if $A$ is the classical Koch curve (i.e., the "snowflake curve" [Md1, pp. 42-43]), Lemma 3.1(i) [or rather, its extension given in (a)] also applies with $N=4, r=1 / 3$, and hence $D(A)=H(A)=\log 4 / \log 3$; this last result will be used in Example 5.4.

(c) In the present paper, we use the word "fractal" to convey the idea of "roughness", without any implication of "self-similarity"; this is in contrast with many authors for whom "fractal" means both "roughness" and "self-similarity" (or, more generally, "self-alikeness"). [In his beautiful recent essays [Md1,2], Mandelbrot does distinguish between "fractals" and "self-similar" or "scaling" "fractals", although almost all of his examples are of the second kind.] In part of the literature, the various notions of "fractal" dimensions are often interchanged without proper justification and even sometimes erroneously. This is the source of much confusion and may be one reason why Berry's conjecture [Be1,2] was initially formulated in terms of the Hausdorff dimension. (See, in particular, Berry's informal scaling argument given in [Be1, p. 52].) Actually, it is only when we freed ourselves from the notion of "self-similarity" that we could see the situation much more clearly and eventually obtain our partial resolution of the Weyl-Berry conjecture. 
Finally, we conclude this section by making explicit some connections with our former (joint) work [FlLa2], in which remainder estimates were obtained for eigenvalue problems involving indefinite weight functions; we mention that in [FlLa2], no use was made of the notion of Minkowski dimension. In order to derive Corollary 3.3 below, we shall use Proposition 3.1 one more time. The following definition is weaker than that of the " $\beta$-condition" introduced in [FILa2, Definition 1, p. 332]. (See Remark 3.5(a) below.)

Definition 3.2. Given $\beta \geq 0$, a bounded subset $A$ of $\mathbb{R}^{n}$ is said to satisfy the (modified) " $\beta$-condition" if \# $K(\varepsilon)=O\left(\varepsilon^{\beta-n}\right)$, as $\varepsilon \rightarrow 0^{+}$, where $K(\varepsilon)=$ $K(\varepsilon ; A)$ is given by $(3.1)$. [Of course, we are only interested here in the case when $\beta \in[0, n]$.]

Corollary 3.3. Let $A \subset \mathbb{R}^{n}$ be bounded. Then $A$ satisfies the (modified) " $\beta$ condition" if and only if $\mathscr{M}_{n-\beta}(A)<+\infty$.

Hence, if $A$ satisfies the (modified) " $\beta$-condition", then $D(A) \geq n-\beta$. Conversely, if $D(A)>n-\beta$ or if $D(A)=n-\beta$ with $\mathscr{M}_{D(A)}<+\infty$, then $A$ satisfies the (modified) " $\beta$-condition".

Proof. First, note that according to Definition 2.1,

$$
\mathscr{M}_{n-\beta}(A):=\limsup _{\varepsilon \rightarrow 0^{+}} \varepsilon^{-\beta}\left|A_{\varepsilon}\right|<+\infty
$$

if and only if $D(A)>n-\beta$ or $D(A)=n-\beta$ with $\mathscr{M}_{D(A)}<+\infty$.

Now, if $\mathscr{M}_{n-\beta}(A)<+\infty$, there exist $\varepsilon_{0}, c>0$ such that $\varepsilon^{-\beta}\left|A_{\varepsilon}\right| \leq c$, $\forall \varepsilon<\varepsilon_{0}$; thus, by the first inequality in (3.2), \#K $\leq c^{\prime} \varepsilon^{\beta-n}, \forall \varepsilon<\varepsilon_{0}$, and so $A$ satisfies the (modified) " $\beta$-condition".

Conversely, if $A$ satisfies the (modified) " $\beta$-condition", there exist $\varepsilon_{0}, c>0$ such that $\# K \leq c \varepsilon^{\beta-n}, \forall \varepsilon<\varepsilon_{0}$; hence, by the second inequality in (3.2), $\left|A_{\varepsilon}\right| \leq c^{\prime} \varepsilon^{\beta}, \forall \varepsilon<\varepsilon_{0}$, and so $\mathscr{M}_{n-\beta}(A)<+\infty$.

Remarks 3.5. (a) It is easy to check that if $A \subset \mathbb{R}^{n}$ obeys the " $\beta$-condition" in the sense of [FILa2, Definition 1, p. 332], then it satisfies the (modified) " $\beta$-condition", in the sense of Definition 3.2 above, for the same value of $\beta$. Moreover, all the results of [FILa2] hold without change if the " $\beta$-condition" is replaced by the weaker (modified) " $\beta$-condition" in the hypotheses of the theorems.

(b) Let $A=\partial \omega$, where $\omega$ is a bounded open subset of $\mathbb{R}^{n}$; this is the case considered in [FlLa2] and of interest to us in the present paper. Then, it follows from Corollaries 3.2 and 3.3 that if $A$ satisfies the (modified) " $\beta$ condition", we must have $\beta \in[0,1]$. [In view of this fact and Corollary 3.1, [FlLa2, Remark 3.1(a), p. 332] must be modified appropriately.] Further, the exponent $\delta$ appearing in the conclusion of [FlLa2, Theorems 1 and $1^{\prime}$, p. 337] is necessarily $\geq 1 / 4 \mathrm{~m}$. Note that the smaller $\beta$, the more "fractal" $\partial \omega$. Moreover, with our present terminology, the "nonfractal" case considered 
in [Mt3] corresponds to the case when $\beta=1$; i.e., $D(\partial \omega)=n-1$ with $\mathscr{M}_{n-1}(\partial \omega)<+\infty$. [Indeed, by Corollary 3.2, $\mathscr{M}_{n-1}(\partial \omega)<+\infty$ if and only if $D(\partial \omega)=n-1$.

(c) In light of the above results and comments, we see a posteriori that some measure of "fractality" was allowed in the hypotheses of [FILa2]. However, it is easily checked that the remainder estimates obtained in [FILa2] (in the special case when the weight function is equal to one and the leading coefficients of $\mathscr{A}$ are locally constant) are much worse than the optimal ones obtained in the present paper. In view of $\S 4$ below, it is clearly possible to extend our results to the case when the operator $\mathscr{A}$ has variable leading coefficients and/or the eigenvalue problem involves an indefinite weight function. However, in order to simplify the exposition, we have chosen to postpone the presentation of this extension to a later work.

(d) For the Neumann problem, the derivation of estimate (41) in [FILa2, p. 346] should be changed. This could be done by using the techniques developed in $\S 4.2$ below. We hope to explain this in more detail in the aforementioned paper.

\section{Proof of the MAIN THEOREM}

The purpose of this section is to establish Theorem 2.1. As was explained in $\S 2.2$, our main results-namely, the remainder estimates for the "counting functions" associated with the Dirichlet and Neumann problems (Corollaries 2.1, 2.2, and Theorem 2.3)-all follow from Theorem 2.1. In addition, we shall see in Appendix A how to derive Theorem 2.2 from Theorem 2.1 and its corollaries.

This section is divided into two parts: in $\S 4.1$, we provide the necessary background and notation, while we prove Theorem 2.1 in $\$ 4.2$. The methods and ideas presented here may also be of interest in future work dealing with both spectral and "fractal" geometry.

We have attempted to give a proof of Theorem 2.1 that was essentially selfcontained, especially for the Dirichlet problem (and second-order operators). The reader who is mainly interested in the Dirichlet Laplacian may assume throughout this section that $\mathscr{A}=-\Delta+1, m=1, V=H_{0}^{1}(\Omega)$, and $a(u, u)=$ $\|u\|_{H^{1}(\Omega)}^{2}$.

4.1. Preliminaries: $i$-widths and eigenvalues. In $\S \S 4.1 . A$ and 4.1.B, we recall the connections between $i$-widths and eigenvalues, as well as their consequences for variational boundary value problems; further, in $\S 4.1$.C, we provide several technical estimates that will be needed in deriving Theorem 2.1.

With the possible exception of $\S 4$.1.C, we suggest that the reader go over the material quickly and then return to it if and when necessary.

4.1.A. $i$-widths. We briefly present some of the basic properties of " $i$-widths". For further information about this subject and its relations with approximation theory, we refer to [Lo or Pn]. 
Definition 4.1. Let $\left(X,\|\cdot\|_{X}\right)$ be a (real or complex) normed linear space and $B$ a subset of $X$. Given any nonnegative integer $i$, the (Kolmogorov) $i$-width (or $i$-diameter) of $B$ in $X$ is given by $d_{i}(B ; X):=\inf _{X_{i}} \sup _{x \in B} \inf _{y \in X_{i}}\|x-y\|_{X}$, where the left-most infimum is taken over all $i$-dimensional subspaces $X_{i}$ of $X$.

Intuitively, the $i$-width $d_{i}(B ; X)$ measures the extent to which $B$ can be approximated by $i$-dimensional subspaces of $X$. [Other notions of $i$-width have been considered in the literature: linear $i$-width, Gelfand $i$-width, etc.; see, e.g., [Pn, especially Chapters I, II, and IV]. It is noteworthy that in the context in which we shall work, all these definitions of $i$-width essentially coincide. (See [Pn, pp. 65-66].)]

We note the following immediate properties (see, e.g., [Pn, Theorem 1.1 and Proposition 1.2, p. 10]).

Lemma 4.1. (a) $d_{i}(B ; X)=d_{i}(\bar{B} ; X)$, where $\bar{B}$ is the closure of $B$ in $X$.

(b) $d_{i}(B ; X)$ increases (resp., decreases) with $B$ (resp., $\left.X\right)$.

(c) The sequence $\left\{d_{i}(B ; X)\right\}_{i=0}^{\infty}$ is nonincreasing. Further, if $B$ is closed and bounded, then $d_{i}(B ; X) \downarrow 0$ if and only if $B$ is compact.

We shall see in the next section how to relate the notion of " $i$-width" with the "counting function" of certain variational eigenvalue problems.

4.1.B. Variational problems and max-min formula. Let $H$ be a (real or complex, infinite-dimensional) Hilbert space, with inner product $(\cdot, \cdot)_{H}$ and norm $\|\cdot\|_{H}$. Let $(W, H, b)$ be a "variational triple"; i.e., $W$ is a dense subspace of $H$ with continuous embedding and $b$ is a bounded, hermitian and coercive form on $W$. (See, e.g., [LiMa, Wb, Mt3, §II or FlLa1, §4].)

We consider the variational eigenvalue problem associated with $(W, H, b)$ :

$$
b(u, v)=\lambda(u, v)_{H} \quad \forall v \in W
$$

here, the scalar $\lambda$ is an eigenvalue of (E) [or is in the "spectrum" of (E)] if there exists a nonzero $u \in W$ such that the latter equation holds.

Example 4.1. We use the notation and hypotheses of $\S 2$. If $H:=L^{2}(\Omega), W:=$ $V$, and $b:=a$, then $(\mathrm{E})$ is simply the variational eigenvalue problem $\left(\mathbf{P}_{\mathscr{A}}\right)$ defined in $\$ 2.2$; recall that by $(2.9), H_{0}^{m}(\Omega) \subset V \subset H^{m}(\Omega)$. In particular, if $W=V:=H_{0}^{m}(\Omega)$ [resp., $W=V:=H^{m}(\Omega)$ ], then (E) is the variational Dirichlet (resp., Neumann) problem $\left(\mathrm{P}_{\mathscr{A}}\right)$.

The hermitian form $b$ induces on $W$ an inner product equivalent to $(\cdot, \cdot)_{H}$. Hence, by the Riesz representation theorem, we can define a bounded positive selfadjoint operator $T$ on the Hilbert space $(W, b(\cdot, \cdot))$ by $b(T u, v)=$ $(u, v)_{H}, \forall u, v \in W$. It follows that $\lambda$ is an eigenvalue of (E) if and only if $\mu:=\lambda^{-1}$ is an eigenvalue of $T$; i.e., $T u=\mu u$, for some nonzero $u$ in $W$.

From now on, we assume that $T$ is compact. This is the case if $W$ is compactly embedded in $H$; this hypothesis holds in all the problems considered in this paper (including, of course, those of Example 4.1). 
Remarks 4.1. (a) It may be helpful to some readers to recall why $T$ is compact under the hypotheses of Example 4.1. For the Dirichlet problem, this follows since the embedding of $H_{0}^{1}(\Omega)$ into $L^{2}(\Omega)$ is compact for any bounded open set $\Omega$; on the other hand, for the Neumann problem, this follows since $H^{1}(\Omega)$ is compactly embedded into $L^{2}(\Omega)$ if $\Omega$ satisfies the " $\left(C^{\prime}\right)$ condition" (a sufficient, but not necessary condition). (See, e.g., [EdEv, Theorem 3.6, p. 227, and $\S 4.9$, pp. 272-274] as well as [M, $\S \S 4.10-4.11$, pp. 249-268].)

(b) Instead of using $T$, one could work with the unbounded selfadjoint operator on $L^{2}(\Omega)$ associated to the coercive form $b$ through the Lax-Milgram lemma, as in [ReSi].

According to the classical theory of compact (positive) selfadjoint operators, the spectrum of $(\mathrm{E})$ is discrete and consists of a sequence $\left\{\mu_{i}\right\}_{i=1}^{\infty}$ of (positive) eigenvalues, written in increasing order according to multiplicity: $(0)<\mu_{1} \leq$ $\mu_{2} \leq \cdots \leq \mu_{i} \leq \cdots$. Further, for $i \geq 1, \mu_{i}^{-1}$ is given by the well known "max-min formula":

$$
\frac{1}{\mu_{i}}=\max _{F_{i} \in \mathscr{F}_{i}} \min _{u \in F_{i}}\left\{(u, u)_{H}: b(u, u) \leq 1\right\},
$$

where $\mathscr{F}_{i}=\mathscr{F}_{i}(W)$ is the set of $i$-dimensional subspaces of $W$. In addition, let $\left\{\phi_{i}\right\}_{i=1}^{\infty}$ be an orthormal basis of $(W, b(\cdot, \cdot))$ of eigenvectors of (E) such that $T \phi_{i}=\mu_{i}^{-1} \phi_{i}$; then the "max-min" in (4.1) is achieved for $F_{i}:=\operatorname{span}\left\{\phi_{1}, \ldots, \phi_{i}\right\}$ and $u:=\phi_{i}$.

We can now provide the basic connection between spectral theory and the notion of " $i$-width" from approximation theory. Set

$$
S_{b}=S_{b}(W)=\{u \in W: b(u, u) \leq 1\} .
$$

Then it results easily from the "max-min formula" (4.1) and the comment following it that

$$
d_{i-1}\left(S_{b}(W) ; H\right)=\left(\mu_{i}\right)^{-1 / 2} \quad \forall i \geq 1,
$$

where $d_{i-1}\left(S_{b}(W) ; H\right)$ is given as in Definition 4.1. (See, e.g., [Pn, Theorems 2.1 and 2.2, pp. 64-66, as well as Remark, p. 66].) Next, for $\lambda>0$, we set as in [Mt1-3]:

$$
N(\lambda ; W, H, b)=\sum_{\lambda d_{i-1}^{2}\left(S_{b}(W) ; H\right) \geq 1} 1=\#\left\{i \geq 0: d_{i}\left(S_{b}(W) ; H\right) \geq \lambda^{-1 / 2}\right\} .
$$

Then, in view of (4.3), we have obtained the following key result:

Proposition 4.1. Let $(W, H, b)$ be a "variational triple" as above. Then, $\forall \lambda>0$,

$$
N(\lambda ; W, H, b)=\sum_{\mu_{i} \leq \lambda} 1=\#\left\{i \geq 1: \mu_{i} \leq \lambda\right\} .
$$


Hence $N(\lambda ; W, H, b)$-as defined in (4.4)-is finite for all $\lambda>0$ and is nothing but the "counting function" (i.e., the number of eigenvalues $\leq \lambda$ counted according to multiplicity) of the variational eigenvalue problem (E).

[Observe that, with the above notation as well as that of $\S 2$ and Example 4.1, we have $N(\lambda)=N\left(\lambda ; V, L^{2}(\Omega), a\right)$.] The next result (see, e.g., [Mt3, $\S 2.2$ or FlLa1, Lemmas 4.2 and 4.3, p. 315]) is an immediate consequence of Lemma 4.1 and Proposition 4.1 - or equivalently, of the "max-min formula" (4.1).

Proposition 4.2 (Monotonicity principles). With the obvious meaning:

(i) $N(\lambda ; W, H, b)$ increases with $W$ and

(ii) $N(\lambda ; W, H, b)$ decreases with $b$.

One also easily deduces from the definitions the following result (see, e.g., [Mt3, Proposition 2.8, pp. 141-142 or FlLa1, 2]):

Lemma 4.2. Let $\left(W_{j}, H_{j}, b_{j}\right), j=1,2$, be variational triples. Set $W=W_{1} \oplus$ $W_{2}, \quad H=H_{1} \oplus H_{2}$ (Hilbert sums), and $b=b_{1} \oplus b_{2}$. Then $(W, H, b)$ is a variational triple and $N(\lambda ; W, H, b)=\sum_{j=1}^{2} N\left(\lambda ; W_{j}, H_{j}, b_{j}\right)$.

Lemma 4.2 in conjunction with Proposition 4.2(i) above, constitutes the basis for the method of "Dirichlet-Neumann bracketing". (See, e.g., [CoHi, ReSi, Mt3, Lal and FlLa1, 2].) Finally, we mention a refinement of this method (as well as of Proposition 4.2(i) and Lemma 4.2) that will be used in the proof of Theorem 2.1. It will provide us with a very convenient tool to obtain upper estimates for the "counting function" on irregular open sets, both for Dirichlet and Neumann boundary conditions. (See [Mt2, Proposition 3, p. 18 or Mt3, Proposition 2.7, p. 138].)

Proposition 4.3. Let $(W, H, b)$ be a variational triple and let $W_{0}$ be a closed subspace of $W$. For $\lambda>0$, let $Z_{\lambda}:=\left\{u \in W: b(u, v)=\lambda(u, v)_{H}, \forall v \in W_{0}\right\}$. Then $N(\lambda ; W, H, b)=N\left(\lambda ; W_{0}, H, b\right)+N\left(\lambda ; Z_{\lambda}, H, b\right)-\operatorname{dim}\left(W_{0} \cap Z_{\lambda}\right)$. Hence, in particular:

$$
N\left(\lambda ; W_{0}, H, b\right) \leq N(\lambda ; W, H, b) \leq N\left(\lambda ; W_{0}, H, b\right)+N\left(\lambda ; Z_{\lambda}, H, b\right) .
$$

The idea of the proof of Proposition 4.3 consists in using an eigenbasis for $b$ and noting the following fact (which results from the "max-min formula" and its equivalent dual form): if $F$ is a closed subspace of $W$ on which the quadratic form $b(\cdot, \cdot)-\lambda(\cdot, \cdot)_{H}$ is negative (resp., positive definite), then its dimension (resp., codimension in $W$ ) is $\leq$ (resp., $\geq$ ) $N(\lambda ; W, H, b)$.

Remark 4.2. We illustrate Proposition 4.3 by the following instructive example: let $W_{0}:=H_{0}^{m}(\omega)$ and $W:=H^{m}(\omega)$, with $\omega \subset \Omega$. Then, in essence, Proposition 4.3 enables us in this context to take into account the contribution to the "counting function" due to the orthogonal complement of $H_{0}^{m}(\omega)$ in $H^{m}(\omega)$.

4.1.C. Technical estimates. We provide here some technical results that will be needed in the proof of Theorem 2.1. 
Besides the classical Sobolev spaces $H_{0}^{m}(\Omega)$ and $H^{m}(\Omega)$, and since the boundary of the open set $\Omega$ is allowed to be irregular, we shall use the following (generalized) Sobolev space:

(4.7)

$\mathscr{H}_{0}^{m}(\Omega):=\left\{u \in H^{m}\left(\mathbb{R}^{n}\right): D^{\alpha} u(x)=0\right.$ for $|\cdot|$-a.e. $x \in \mathbb{R}^{n} \backslash \Omega$, and for $\left.|\alpha| \leq m\right\}$.

[As before, $|\cdot|=|\cdot|_{n}$ denotes $n$-dimensional Lebesgue measure.] Naturally, in (4.7), $D^{\alpha} u$ stands for the distributional (or "weak") derivative of $u$. Clearly, $H_{0}^{m}(\Omega) \subset \mathscr{H}_{0}^{m}(\Omega) \subset H^{m}(\Omega)$. More precisely, $\mathscr{H}_{0}^{m}(\Omega)$ is a closed subspace of $H^{m}\left(\mathbb{R}^{n}\right)$ that we identify with a closed subspace of $H^{m}(\Omega)$ containing $H_{0}^{m}(\Omega)$. [Note that the space $\mathscr{H}_{0}^{m}(\Omega)$ enables us to give a precise meaning to the phrase: "extend the function $u$ by 0 outside $\Omega$." (Of course, other function spaces could also be used for this purpose.)]

Let $a^{\prime}$ be the leading form associated with the leading part $\mathscr{A}^{\prime}$ of $\mathscr{A}$, and let $a_{\omega}^{\prime}$ be its restriction to $\omega \subset \Omega$ (i.e., $\left.a_{\omega}^{\prime}(u, u):=\int_{\omega} \sum_{|\alpha|=|\beta|=m} a_{\alpha \beta}(x) D^{\alpha} u D^{\beta} \bar{u}\right)$. The following result-the proof of which makes use of Proposition 4.3 (see Remark 4.2 above) -is obtained by combining [Mt3, Proposition 4.1, p. 162 and FlLa2, Lemma 2, p. 352, and Appendix, p. 354]. It yields in particular a uniform remainder estimate on cubes of the same size, for the "counting functions" associated with the Dirichlet and Neumann problems.

Proposition 4.4. Assume that hypotheses (2.9) through (2.11) hold (so that, in particular, $\mathscr{A}^{\prime}$ is a homogeneous operator of order $2 \mathrm{~m}$ with (locally) constant coefficients on $\Omega$ ). Then there exists a positive constant $c$ such that for all $\varepsilon>0$, all (open n-dimensional) cubes $Q \subset \Omega$ of side $\varepsilon$ and all $\lambda, \nu>0$, we have:

(i) $\left|N\left(\lambda ; W_{Q}, L^{2}(Q), a_{Q}^{\prime}\right)-\mu_{\mathscr{Q}}^{\prime}(Q) \lambda^{n / 2 m}\right| \leq c\left[1+\varepsilon^{n-1} \lambda^{(n-1) / 2 m}\right]$, where $W_{Q}$ denotes any one of the spaces $H_{0}^{m}(Q), \mathscr{B}_{0}^{m}(Q)$, or $H^{m}(Q)$.

(ii) $N\left(\nu ; Z_{\lambda}(Q), L^{2}(Q), a_{Q}^{\prime}\right) \leq c\left[1+\varepsilon^{n-1}\left(\lambda^{(n-1) / 2 m}+\nu^{(n-1) / 2 m}\right)\right]$, where $Z_{\lambda}(Q):=\left\{u \in H^{m}(Q): a_{Q}^{\prime}(u, v)=\lambda(u, v)_{L^{2}(Q)}, \forall v \in H_{0}^{m}(Q)\right\}, b y$ analogy with Proposition 4.3.

Remarks 4.3. (a) Part (i) [for $W_{Q}$ equal to $H_{0}^{1}(Q)$ or $H^{1}(Q)$, respectively] is well known when $\mathscr{A}=\mathscr{A}^{\prime}=-\Delta$; in this case, it is obtained by an explicit calculation of the eigenvalues on the cube $Q$ for the Dirichlet or Neumann problem, respectively. (See, e.g., [CoHi, $\S \mathrm{VI} .1$ and $\mathrm{ReSi}$, Proposition 2, pp. 266-267].)

(b) The constant $c$ in the conclusion of Proposition 4.4 depends only on $M:=\max _{|\alpha|=|\beta|=m}\left\|a_{\alpha \beta}\right\|_{L^{\infty}(\Omega)}$ and the constant of uniform ellipticity of $\mathscr{A}$; this follows since, according to hypothesis (2.11), the coefficients of $\mathscr{A}^{\prime}$ lie in $L^{\infty}(\Omega)$ and are constant on each open cube $Q \subset \Omega$.

The next result [Mt3, Lemma 3.3, p. 150] will be used in the proof of Proposition 4.5 below. 
Lemma 4.3. Let $U$ be a convex bounded open set in $\mathbb{R}^{n}$ of diameter diam $U$ and let $U_{1}, U_{2}$ be measurable subsets of $U$. Then, for all $f \in H^{1}(U)$, we have:

$$
\iint_{U_{1} \times U_{2}}|f(x)-f(y)|^{2} d y d x \leq 2^{n-1}\left(\left|U_{1}\right|+\left|U_{2}\right|\right)[\operatorname{diam} U]^{2}\|f\|_{H^{1}(U)}^{2} .
$$

Proof. By a density argument (see, e.g., [EdEv, Theorem 3.2, p. 221]), we may assume that $f \in H^{1}(U) \cap C^{1}(U)$. Further, for $(x, y) \in U_{1} \times U_{2}$ and by the convexity of $U$, we can write

$$
f(y)-f(x)=\int_{0}^{1}\langle\nabla f(t x+(1-t) y), x-y\rangle d t,
$$

where $\langle\cdot, \cdot\rangle$ is the inner product in $\mathbb{R}^{n}$ and $\nabla f$ the gradient of $f$; hence Hölder's inequality yields

$$
|f(x)-f(y)|^{2} \leq[\operatorname{diam} U]^{2} \int_{(0,1)}|\nabla f(t x+(1-t) y)|^{2} d t .
$$

We now integrate this inequality over $U_{1} \times U_{2}$, break $(0,1)$ into $(0,1 / 2)$ and $(1 / 2,1)$, and perform the change of variables $\kappa(x, y, t):=(x, t x+(1-t) y, t)$ [resp., : $=(t x+(1-t) y, y, t)]$ for $t<1 / 2$ [resp., $>1 / 2$ ]; the conclusion then follows since $\|\nabla f\|_{L^{2}(U)}^{2} \leq\|f\|_{H^{1}(U)}^{2}$.

We shall use in Proposition 4.5 (resp., 4.5') the following abstract definition when $W=\mathscr{H}_{0}^{m}(\Omega)$ [resp., $=H^{m}(\Omega)$ ], equipped with the usual Sobolev norm $\|\cdot\|_{H^{m}(\Omega)}$.

Definition 4.2. Let the (Hilbert) space $W$ be continuously embedded in $L^{2}(\Omega)$ and let $\omega$ be an open subset of $\Omega$. Let $S(W)$ be the closed unit ball of $W$ (for its original norm) and $S(W)_{/ \omega}$ the set of restrictions to $\omega$ of elements of $S(W)$. Let $d_{i}\left(S(W)_{/ \omega} ; L^{2}(\omega)\right)$, the $i$-width of $S(W)_{/ \omega}$ in $L^{2}(\omega)$, be given as in Definition 4.1. Then, for $\lambda>0$, set [by analogy with (4.4)]:

$$
N^{*}\left(\lambda ; W, L^{2}(\omega)\right)=\#\left\{i \geq 0: d_{i}\left(S(W)_{/ \omega} ; L^{2}(\omega)\right) \geq \lambda^{-1 / 2}\right\} .
$$

Remarks 4.4. (a) It follows from Proposition 4.1 that (with the notation of $\S \S 2$ and 4.1.B) when $\omega:=\Omega$ and $W:=V$ is equipped with the Hilbert norm $a(\cdot, \cdot): N^{*}\left(\lambda ; V, L^{2}(\Omega)\right)=N\left(\lambda ; V, L^{2}(\Omega), a\right)=N(\lambda)$.

(b) When $W$ is one of the aforementioned Sobolev spaces, one deduces easily from the definitions and Lemma $4.1(\mathrm{~b})$ that $N^{*}\left(\lambda ; W, L^{2}(\omega)\right)$ increases with the space $W$, as well as with the open set $\omega$. (For this latter fact, see [Mt3, Eq. (3.3), p. 147].)

The next result [Mt3, Proposition 3.4, p. 151] will enable us to obtain boundary estimates for the Dirichlet problem in the proof of Theorem 2.1; it will thus be of interest when $\partial \omega \cap \partial \Omega \neq \varnothing$. 
Proposition 4.5. Let $\Omega$ be an arbitrary bounded open set in $\mathbb{R}^{n}$ and let $\omega$ be an open subset of $\Omega$. Then there exists a positive constant $C$-depending only on $n$ and $m$-such that for all $\lambda>0$ :

$$
N^{*}\left(\lambda ; \mathscr{H}_{0}^{m}(\Omega), L^{2}(\omega)\right) \leq C\left|\omega_{\sqrt{n} \lambda^{-1 / 2 m}} \cap \Omega\right| \lambda^{n / 2 m} .
$$

Here, $\omega_{\varepsilon}=\left\{x \in \mathbb{R}^{n}: d(x, \omega)<\varepsilon\right\}$ is given as in equation (2.1) and $N^{*}\left(\lambda ; \mathscr{H}_{0}^{m}(\Omega), L^{2}(\omega)\right)$ is defined as in (4.8).

As was pointed out in [Mt3], the proof relies on methods from approximation theory ( $i$-widths estimates in Sobolev spaces) used, in particular, in [BiSo and Ek] (see also [Pn, Chapter VII]); we include it in the basic case when $m=1$ in order to keep the derivation of Theorem 2.1 (essentially) self-contained for the Dirichlet problem.

Proof of Proposition 4.5. Assume that $m=1$. [The case when $m \geq 2$ is deduced from that when $m=1$ by a bootstrap argument (and a suitable extension of Green's formula); see [Mt3, Proposition 3.1, p. 148].] Given $\varepsilon>0$, let $\left\{Q_{\zeta}\right\}_{\zeta \in \mathbb{Z}^{n}}$ be a tessellation of $\mathbb{R}^{n}$ by disjoint congruent open cubes of side $\varepsilon$ (and hence volume $\left|Q_{\zeta}\right|=\varepsilon^{n}$ ). Let $K:=\left\{\zeta \in \mathbb{Z}^{n}: Q_{\zeta} \cap \omega \neq \varnothing\right\}$ and let $K^{\prime} \subset K$ be defined by $K^{\prime}=\left\{\zeta \in K:\left|Q_{\zeta} \cap \Omega\right| \geq \frac{1}{2}\left|Q_{\zeta}\right|\right\}$. Set $\omega^{\prime}=\operatorname{Int}\left[\bigcup_{\zeta \in K^{\prime}}\left(\overline{Q_{\zeta}} \cap \Omega\right)\right]$; then, clearly, $\omega \subset \omega^{\prime} \subset \omega_{\sqrt{n} \varepsilon} \cap \Omega$ and $\frac{1}{2} \varepsilon^{n}\left(\# K^{\prime}\right) \leq\left|\omega^{\prime}\right|$. Thus

$$
i:=\# K^{\prime} \leq 2\left|\omega_{\sqrt{n} \varepsilon} \cap \Omega\right| \varepsilon^{-n} .
$$

Let $f \in \mathscr{H}_{0}^{1}(\Omega)$. Recall that by definition [see (4.7)], $f$ extends to $\tilde{f} \in$ $H^{1}\left(\mathbb{R}^{n}\right)$ such that $\tilde{f}=0$ and $\nabla \tilde{f}=0|\cdot|$-a.e. in $\mathbb{R}^{n} \backslash \Omega$. On each $Q_{\zeta} \cap \Omega$, we shall approximate $f$ by the mean value of $\tilde{f}$ on $Q_{\zeta}$ (for $\zeta \in K^{\prime}$ ), and by 0 (for $\zeta \in K \backslash K^{\prime}$ ). More precisely, we set, for $\zeta \in K^{\prime}, f_{\zeta}=\frac{1}{\left|Q_{\xi}\right|} \int_{Q_{\xi}} \tilde{f}(x) d x$; then

$$
\left\|f-f_{\zeta}\right\|_{L^{2}\left(Q_{\xi} \cap \Omega\right)}^{2} \leq \frac{1}{\left|Q_{\zeta}\right|} \int_{Q_{\xi} \times Q_{;}}|\tilde{f}(x)-\tilde{f}(y)|^{2} d y d x .
$$

We now apply Lemma 4.3 (with $U_{1}=U_{2}=U:=Q_{\zeta}$ ) and note that $\|\tilde{f}\|_{H^{1}\left(Q_{\xi}\right)}=$ $\|f\|_{H^{1}\left(Q_{\xi} \cap \Omega\right)}$ to obtain

$$
\left\|f-f_{\zeta}\right\|_{L^{2}\left(Q_{\xi} \cap \Omega\right)}^{2} \leq n 2^{n} \varepsilon^{2}\|f\|_{H^{\prime}\left(Q_{\xi} \cap \Omega\right)}^{2} \quad \forall \zeta \in K^{\prime} .
$$

Similarly, with $\Omega^{c}:=\mathbb{R}^{n} \backslash \Omega$ and for $\zeta \in K \backslash K^{\prime}$, we write

$$
\|f\|_{L^{2}\left(Q_{\xi} \cap \Omega\right)}^{2}=\frac{1}{\left|Q_{\zeta} \cap \Omega^{c}\right|} \int_{\left(Q_{\xi} \cap \Omega\right) \times\left(Q_{\xi} \cap \Omega^{c}\right)}|\tilde{f}(x)-\tilde{f}(y)|^{2} d y d x ;
$$

hence, by Lemma 4.3 (with $U_{1}:=Q_{\zeta} \cap \Omega, U_{2}:=Q_{\zeta} \cap \Omega^{c}$, and $U:=Q_{\zeta}$ ) and since for $\zeta \in K \backslash K^{\prime},\left|Q_{\zeta} \cap \Omega^{c}\right|>\left|Q_{\zeta} \cap \Omega\right|$, we have:

$$
\|f\|_{L^{2}\left(Q_{\xi} \cap \Omega\right)}^{2} \leq n 2^{n} \varepsilon^{2}\|f\|_{H^{\prime}\left(Q_{;} \cap \Omega\right)}^{2} \quad \forall \zeta \in K \backslash K^{\prime} .
$$


By combining (4.11) and (4.12), we deduce that given any $f \in \mathscr{H}_{0}^{1}(\Omega)$, with $\|f\|_{H^{1}(\Omega)} \leq 1$, there exists $g:=\sum_{\zeta \in K^{\prime}} f_{\zeta} 1_{Q_{\zeta}}$ such that

$$
\|f-g\|_{L^{2}(\omega)}^{2}\left(\leq n 2^{n} \varepsilon^{2}\|f\|_{H^{1}\left(\omega^{\prime}\right)}^{2}\right) \leq n 2^{n} \varepsilon^{2} .
$$

It now suffices to consider the $i$-dimensional subspace of $L^{2}\left(\omega^{\prime}\right)$ spanned by the characteristic functions $1_{Q_{\zeta}}, \zeta \in K^{\prime}$ (where $i:=\# K^{\prime}$ ), to infer from Definition 4.1 that

$$
d_{i}^{2}\left(S\left(\mathscr{H}_{0}^{1}(\Omega)\right)_{/ \omega^{\prime}} ; L^{2}\left(\omega^{\prime}\right)\right) \leq n 2^{n} \varepsilon^{2}
$$

Consequently, for $0<\lambda \leq n^{-1} 2^{-n} \varepsilon^{-2}$,

$$
N^{*}\left(\lambda ; \mathscr{H}_{0}^{1}(\Omega), L^{2}(\omega)\right) \leq N^{*}\left(\lambda ; \mathscr{H}_{0}^{1}(\Omega), L^{2}\left(\omega^{\prime}\right)\right) \leq i \leq 2\left|\omega_{\sqrt{n} \varepsilon} \cap \Omega\right| \varepsilon^{-n} .
$$

[The first inequality in (4.15) follows from Remark 4.4(b) since $\omega \subset \omega^{\prime}$; the second results from (4.14) and Definition 4.2, while the third one is just (4.10).] By choosing, for a given $\lambda>0, \varepsilon=n^{-1 / 2} 2^{-n / 2} \lambda^{-1 / 2}$, we obtain (4.9) with $m=1$, as desired.

The following result [Mt3, Proposition 3.8, p. 158] (see Remark 4.5(b))which is the counterpart of Proposition 4.5 for the Sobolev space $H^{m}(\Omega)$-will be used to obtain boundary estimates for the Neumann problem.

Proposition $4.5^{\prime}$. Let $\Omega$ be a bounded open set in $\mathbb{R}^{n}$ that satisfies the " $\left(\mathrm{C}^{\prime}\right)$ condition". Then there exist positive constants $\lambda_{0}^{\prime}, c^{\prime}$ and $C$ such that for all $\varepsilon>0$ and all $\lambda \geq \lambda_{0}^{\prime}$ :

$$
N^{*}\left(\lambda ; H^{m}(\Omega), L^{2}\left(\widetilde{\Gamma}_{\varepsilon}\right)\right) \leq C\left|\Gamma_{\varepsilon+c^{\prime} \lambda^{-1 / 2 m}}\right| \lambda^{n / 2 m} .
$$

Here, $\Gamma=\partial \Omega, \widetilde{\Gamma}_{\varepsilon}=\{x \in \Omega: d(x, \Gamma)<\varepsilon\}$, as in (2.5), and $\Gamma_{\delta}=\left\{x \in \mathbb{R}^{n}\right.$ : $d(x, \Gamma)<\delta\}$, as in $(2.1)$.

Remarks 4.5. (a) Proposition $4.5^{\prime}$ is proved in much the same way as Proposition 4.5 by means of $i$-width estimates and (a variant of) Lemma 4.3 once an open cover adapted to the " $\left(C^{\prime}\right)$ condition" (see Definition 2.2 ) is chosen.

(b) Actually, according to the conclusion of [Mt3, Proposition 3.8, p. 158] (and with our notation), the right-hand side of (4.16) should be equal to $C\left|\left(\widetilde{\Gamma}_{\varepsilon}\right)_{\delta}\right|$ with $\delta:=c^{\prime} \lambda^{-1 / 2 m}$. However, one easily checks (see the comment following (4.23a) below) that $\left(\widetilde{\Gamma}_{\varepsilon}\right)_{\delta} \subset \Gamma_{\varepsilon+\delta}$, from which $(4.16)$-in the form we have written it-follows immediately.

4.2. Proof of Theorem 2.1. We now establish Theorem 2.1. We first consider the Dirichlet problem [i.e., $V=H_{0}^{m}(\Omega)$ ] in $\S 4.2$.A and then the Neumann [i.e., $V=H^{m}(\Omega)$ ] (or, more generally, mixed) problem in $\S 4.2$.B. In this latter case, the proof will follow similar lines except in a few significant instances which we will point out. 
4.2.A. The Dirichlet problem. We suppose here that the hypotheses of Theorem 2.1 for Dirichlet boundary conditions are satisfied. Hence hypotheses $(2.10)$ and (2.11) hold with $V=H_{0}^{m}(\Omega)$; further, $\Omega$ is a nonempty bounded open set of $\mathbb{R}^{n}$ such that $\widetilde{\mathscr{M}}_{d}(\Gamma)<+\infty$ for some $d \in[n-1, n]$. [Recall that $\Gamma=\partial \Omega$ denotes the boundary of $\Omega$ and that $\widetilde{\mathscr{M}}_{d}(\Gamma)$-the $d$-dimensional upper Minkowski content of $\Gamma$, relative to $\Omega$-is defined in (2.6).]

We now outline the main steps in the proof of Theorem 2.1:

By using a refinement of the method of "Dirichlet-Neumann bracketing" for irregular open sets, we obtain an estimate for the "counting function" $N(\lambda)$, that involves an "interior term" and a "boundary term". By localization on cubes, the "interior term" gives rise to the leading term $\phi(\lambda)$ in Weyl's asymptotic formula, and a "remainder term" $R(\lambda)$; the latter one and the above "boundary term" can be estimated in function of the "fractality" of the boundary $\Gamma$.

More precisely, by suitably choosing tessellations of $\mathbb{R}^{n}$ into small cubes whose size tends to zero as you approach $\Gamma$ (and hence inducing Whitneytype coverings of $\Omega$ ), we show that the "boundary term" is $O\left(\lambda^{d / 2 m}\right)$, as $\lambda \rightarrow$ $+\infty$, and that the same is true of $R(\lambda)$ except possibly when $d=n-1$, the topological dimension of $\Gamma$; in this latter case, we show instead that $R(\lambda)$ is $O\left(\lambda^{d / 2 m} \log \lambda\right)$.

We conclude, as desired, that Weyl's asymptotic formula holds with a remainder that is $O\left(\lambda^{d / 2 m}\right)$ in the "fractal" case when $d>n-1$, and $O\left(\lambda^{d / 2 m} \log \lambda\right)$ when $d=n-1$.

Different versions of the proof of Theorem 2.1 can be adopted; however, in essence, they can all be described as above.

It will be convenient to use the following notation: for $\lambda>0$ and $\omega$ open, $\omega \subset \Omega$, we set

$$
N_{0}(\lambda ; \omega)=N\left(\lambda ; H_{0}^{m}(\omega), L^{2}(\Omega), a\right) .
$$

[We refer to Proposition 4.1 for the precise definition of the right-hand side of (4.17); see (4.4) and (4.5).] Note that, in particular, $N(\lambda)=N_{0}(\lambda ; \Omega)$. (See Example 4.1.) Intuitively, $N_{0}(\lambda ; \omega)$ can be thought of as the "counting function" associated with a realization of the variational eigenvalue problem: $\mathscr{A} u=\lambda u$, on $\omega$, with Dirichlet boundary conditions. Moreover, we let

$$
\begin{aligned}
& N_{0}^{*}(\lambda ; \omega)=N^{*}\left(\lambda ; H_{0}^{m}(\Omega), L^{2}(\omega)\right) \text { and } \\
& N_{1}^{*}(\lambda ; \omega):=N^{*}\left(\lambda ; \mathscr{H}_{0}^{m}(\Omega), L^{2}(\omega)\right) .
\end{aligned}
$$

[Here, the right-hand side of each equality in (4.18) is given as in (4.8); see Definition 4.2. Further, $\mathscr{H}_{0}^{m}(\Omega)$ is the Sobolev space introduced in $\S 4$.1.C; see (4.7).] If $\mu_{\mathscr{A}}^{\prime}(\omega)$ is defined as in (2.18) [with $\Omega$ replaced by $\omega$ ], we also set

$$
\phi(\lambda ; \omega)=\mu_{\mathscr{A}}^{\prime}(\omega) \lambda^{n / 2 m} \text { and } \phi(\lambda)=\phi(\lambda ; \Omega) .
$$

[Recall for later use that the (Browder-Gårding) measure $\mu_{\mathscr{A}}^{\prime}(\cdot)$ is absolutely continuous with respect to Lebesgue measure $|\cdot|_{n}$. (For example, if $\mathscr{A}=$ $-\Delta+1$, then $\left.\left.\mu_{\mathscr{A}}^{\prime}(\omega)=(2 \pi)^{-n} \mathscr{B}_{n}|\omega|_{n}.\right)\right]$ 
With these notations, the conclusion of Theorem 2.1 reads as follows:

$$
|N(\lambda)-\phi(\lambda)|=O\left(\lambda^{d / 2 m}\right)
$$

[resp., $O\left(\lambda^{d / 2 m} \log \lambda\right)$ ], as $\lambda \rightarrow+\infty$, when $d>n-1$ [resp., $=n-1$ ].

The proof of (4.20) [and hence of Theorem 2.1] is divided into four steps.

STEP 1. The following key result holds:

Proposition 4.6 (Estimate near the boundary). There exist positive constants $\varepsilon_{0}, \lambda_{0}$, and $C_{0}$ such that for all positive $\varepsilon<\varepsilon_{0}$ and all $\lambda \geq \lambda_{0} \varepsilon^{-2 m}$, we have

$$
N_{1}^{*}\left(\lambda ; \widetilde{\Gamma}_{\varepsilon}\right) \leq C_{0} \varepsilon^{n-d} \lambda^{n / 2 m} .
$$

Here, $\widetilde{\Gamma}_{\varepsilon}=\{x \in \Omega: d(x, \Gamma)<\varepsilon\}=\Gamma_{\varepsilon} \cap \Omega$ denotes the open $\varepsilon$-neighborhood of $\Gamma$, relative to $\Omega$, as in (2.5); further, $N_{1}^{*}\left(\lambda ; \widetilde{\Gamma}_{\varepsilon}\right)$ is given as in (4.18).

Proof. First, fix $\varepsilon>0$. Apply Proposition 4.5-which as we recall, relies on the method of $i$-width from approximation theory and Lemma 4.3-with $\omega:=\widetilde{\Gamma}_{\varepsilon}$. Hence, in view of (4.18), we deduce that there exists a positive constant $C$ such that

$$
N_{1}^{*}\left(\lambda ; \widetilde{\Gamma}_{\varepsilon}\right) \leq C\left|\left(\widetilde{\Gamma}_{\varepsilon}\right)_{\delta} \cap \Omega\right| \lambda^{n / 2 m} \quad \forall \lambda>0,
$$

with $\delta:=\sqrt{n} \lambda^{-1 / 2 m}$ and $A_{\delta}=\left\{x \in \mathbb{R}^{n}: d(x, A)<\delta\right\}$ defined as in (2.1). Now, we note that $\left(\widetilde{\Gamma}_{\varepsilon}\right)_{\delta} \cap \Omega \subset \widetilde{\Gamma}_{\varepsilon+\delta}$ and hence

$$
\left|\left(\widetilde{\Gamma}_{\varepsilon}\right)_{\delta} \cap \Omega\right| \leq\left|\widetilde{\Gamma}_{\varepsilon+\delta}\right| .
$$

[Indeed, it easily follows from the definitions that for $A \subset B \subset \mathbb{R}^{n}$, we have $A_{\varepsilon} \subset B_{\varepsilon}$ and $\left(B_{\varepsilon}\right)_{\delta} \subset B_{\varepsilon+\delta}$. Thus, since $\widetilde{\Gamma}_{\varepsilon} \subset \Gamma_{\varepsilon}$, we obtain $\left(\widetilde{\Gamma}_{\varepsilon}\right)_{\delta} \subset\left(\Gamma_{\varepsilon}\right)_{\delta} \subset$ $\Gamma_{\varepsilon+\delta}$ and hence $\left(\tilde{\Gamma}_{\varepsilon}\right)_{\delta} \cap \Omega \subset \Gamma_{\varepsilon+\delta} \cap \Omega=\widetilde{\Gamma}_{\varepsilon+\delta}$.]

Next, since, by assumption, $\widetilde{\mathscr{M}}_{d}(\Gamma):=\lim \sup _{\varepsilon \rightarrow 0^{+}} \varepsilon^{-(n-d)}\left|\widetilde{\Gamma}_{\varepsilon}\right|<+\infty$, we deduce that there exist positive constants $\varepsilon_{0}^{\prime}$ and $c$ such that

$$
\left|\widetilde{\Gamma}_{\varepsilon}\right| \leq c \varepsilon^{n-d} \quad \forall \varepsilon<\varepsilon_{0}^{\prime} .
$$

Finally, we take any $\lambda_{0}>0$ and set $\varepsilon_{0}=\varepsilon_{0}^{\prime}\left[1+\sqrt{n}\left(\lambda_{0}\right)^{-1 / 2 m}\right]^{-1}$; then, for $\varepsilon<\varepsilon_{0}$ and $\lambda \geq \lambda_{0} \varepsilon^{-2 m}$, we have $\varepsilon+\delta:=\varepsilon+\sqrt{n} \lambda^{-1 / 2 m} \leq\left[1+\sqrt{n}\left(\lambda_{0}\right)^{-1 / 2 m}\right] \varepsilon<$ $\varepsilon_{0}^{\prime}$. Thus, we conclude from (4.22), (4.23a), and (4.23b) that $N_{1}^{*}\left(\lambda ; \tilde{\Gamma}_{\varepsilon}\right) \leq$ $C\left|\widetilde{\Gamma}_{\varepsilon+\delta}\right| \lambda^{n / 2 m} \leq C_{0} \varepsilon^{n-d} \lambda^{n / 2 m}$, as desired.

Remark 4.6. The counterpart of Proposition 4.6-with the Sobolev space $H^{m}(\Omega)$ instead of $\mathscr{H}_{0}^{m}(\Omega)$-still holds for the Neumann problem provided that hypothesis (2.13) [the " $\left(C^{\prime}\right)$ condition”] is assumed. (See $\S 4$.2.B.) We must then use Proposition 4.5' instead of Proposition 4.5 and require that $\mathscr{M}_{d}(\Gamma)$ [and not just $\left.\widetilde{\mathscr{M}_{d}}(\Gamma)\right]$ is finite. This is the place in the proof of Theorem 2.1 (and its 
corollaries) where appears naturally the distinction between the Minkowski dimension $D$ (for the Neumann problem) and the relative Minkowski dimension $\widetilde{D}$ (for the Dirichlet problem).

STEP 2. We construct as in [CoHi, §VI.5, pp. 443-445 or Mt3, §VI.3, pp. 197-199] a sequence of tessellations $\left\{Q_{\zeta}^{p}\right\}_{\zeta \in \mathbb{Z}^{n}}, p=0,1,2, \ldots$, having the following properties:

For each nonnegative integer $p,\left\{Q_{\zeta}^{p}\right\}_{\zeta \in \mathbb{Z}^{n}}$ is a tessellation of $\mathbb{R}^{n}$ into a countable family of congruent and nonoverlapping open ( $n$-dimensional) cubes of side $\varepsilon_{p}:=2^{-p}$ such that $\bigcup_{\zeta \in \mathbb{Z}^{n}} \overline{Q_{\zeta}^{p}}=\mathbb{R}^{n}$. Further, the cubes of the $p$ th "generation" are obtained by halving the sides of each cube of the previous generation.

We shall use these tessellations to approximate the open set $\Omega$ as well as its boundary $\Gamma$. Hence, we define (by induction on $p$ ) the following index set $I_{p}$ and open subsets $\Omega_{p}^{\prime}, \omega_{p}$ of $\Omega:$ if $I_{0}:=\left\{\zeta \in \mathbb{Z}^{n}: Q_{\zeta}^{0} \subset \Omega\right\}$, we set $\Omega_{0}^{\prime}=\bigcup_{\zeta \in I_{0}} Q_{\zeta}^{0}$ and $\omega_{0}=\Omega \backslash \overline{\Omega_{0}^{\prime}}$. Furthermore, $I_{1}:=\left\{\zeta \in \mathbb{Z}^{n}: Q_{\zeta}^{1} \subset \omega_{0}\right\}=$ $\left\{\zeta \in \mathbb{Z}^{n}: Q_{\zeta}^{1} \subset \Omega\right.$ and $\left.\zeta \notin I_{0}\right\}, \Omega_{1}^{\prime}:=\Omega_{0}^{\prime} \cup\left(\bigcup_{\zeta \in I_{1}} Q_{\zeta}^{1}\right)$, and $\omega_{1}:=\Omega \backslash \overline{\Omega_{1}^{\prime}}$; and so on. More precisely, if $p \geq 1$ and we have defined $I_{q}, \Omega_{q}^{\prime}$, and $\omega_{q}$ for $q \leq p-1$, we let

$$
I_{p}:=\left\{\zeta \in \mathbb{Z}^{n}: Q_{\zeta}^{p} \subset \omega_{p-1}\right\}=\left\{\zeta \in \mathbb{Z}^{n}: Q_{\zeta}^{p} \subset \Omega \text { and } \zeta \notin \bigcup_{q=1}^{p-1} I_{q}\right\}
$$

moreover, we set

$$
\Omega_{p}^{\prime}=\Omega_{p-1}^{\prime} \cup\left(\bigcup_{\zeta \in I_{p}} Q_{\zeta}^{p}\right) \quad \text { and } \quad \omega_{p}=\Omega \backslash \overline{\Omega_{p}^{\prime}} .
$$

The reader may find it helpful to represent graphically some of the sets involved in this construction. [Obviously, $\Omega_{p}, \omega_{p}$ do not stand here for $\varepsilon$ neighborhoods; this notation should not create any confusion since the subscript $p$ will always be an integer.]

Note that by construction, the $I_{q}$ 's are pairwise disjoint and hence the following disjoint union holds for $p \geq 1$ :

$$
\Omega_{p}^{\prime}=\bigcup_{q=0}^{p}\left(\bigcup_{\zeta \in I_{q}} Q_{\zeta}^{q}\right) \text {. }
$$

Moreover, we observe that for all $p \geq 1$ :

$$
\omega_{p} \subset \widetilde{\Gamma}_{c_{1} \varepsilon_{p}},
$$

with $c_{1}:=1+\sqrt{n}$ (or, more precisely, any constant $c_{1}>\sqrt{n}$ ) and $\varepsilon_{p}=2^{-p}$.

Indeed, let $x \in \omega_{p}$; then $x \in \overline{Q_{\zeta}^{p}}$ for some $\zeta$. Clearly, by (4.24b), $\overline{Q_{\zeta}^{p}} \cap \Gamma \neq$ $\varnothing$ and hence $d(x, \Gamma)<c_{1} \varepsilon_{p}$; since also $x \in \Omega$, we conclude that $x \in \widetilde{\Gamma}_{c_{1} \varepsilon_{p}}$. 
Throughout the end of the present step and Step 3, we shall work with $p$ fixed sufficiently large. Then, in the last step of the proof of Theorem 2.1, we shall make a particular choice of $p$ in a function of $\lambda$ in order to optimize our estimates.

Convention. In the rest of this section, positive real (resp., integral) constants will be denoted by $\lambda_{1}, c_{0}, c_{1}^{\prime}, C_{1}, \ldots$ (resp., $\left.p_{1}, p_{2}, p_{1}^{\prime}, \ldots\right)$; they will depend only on (all or possibly some of) $n, d, m, \Omega$, and $\mathscr{A}$.

Proposition 4.7. There exist positive constants $\lambda_{1}>1, C_{1}$, and $p_{1}$ such that for all integers $p \geq p_{1}$ and all $\lambda \geq \lambda_{1}\left(\varepsilon_{p}\right)^{-2 m}$, we have

$$
|N(\lambda)-\phi(\lambda)| \leq C_{1}\left[\left(\varepsilon_{p}\right)^{n-d} \lambda^{n / 2 m}+R_{p}(\lambda)\right],
$$

where

$$
R_{p}(\lambda):=\sum_{q=0}^{p}\left(\# I_{q}\right)\left[1+\left(\varepsilon_{q}\right)^{n-1} \lambda^{(n-1) / 2 m}\right] .
$$

Proof. Fix $p \geq 2$ and $\lambda>0$. For notational simplicity, we set $\Omega^{\prime}=\Omega_{p}^{\prime}$ and $\omega=\omega_{p}$, where $\Omega_{p}^{\prime}, \omega_{p}$ are given by $(4.24 \mathrm{~b})$ above; recall that $\Omega^{\prime}$ and $\omega$ are disjoint open subsets of $\Omega$. Intuitively, $\Omega^{\prime}$ (resp., $\omega$ ) can be thought of as approximating the open set $\Omega$ (resp., the boundary $\Gamma$ ) "from within" $\Omega$.

We use a refinement of the method of "Dirichlet-Neumann bracketing" for irregular open sets (see, e.g., [Mt3; FlLa1, Lemma 5.2, p. 317; FlLa2], and in the classical case, [CoHi, Chapter VI or ReSi, Propositions 3 and 4, pp. 269-270]). Specifically, we apply Proposition 4.3 , with $W_{0}:=H_{0}^{m}\left(\Omega^{\prime}\right)$ and $W:=H_{0}^{m}(\Omega)$ [as well as $H:=L^{2}(\Omega)$ and $b:=a$ ], to deduce that

$$
N_{0}\left(\lambda ; \Omega^{\prime}\right) \leq N(\lambda) \leq N_{0}\left(\lambda ; \Omega^{\prime}\right)+\mathscr{N}(\lambda ; \omega),
$$

where

$$
\mathscr{N}(\lambda ; \omega):=N\left(\lambda ; Z_{\lambda}, L^{2}(\Omega), a\right)
$$

and

$$
Z_{\lambda}:=\left\{u \in H_{0}^{m}(\Omega): a(u, v)=\lambda(u, v)_{L^{2}(\Omega)}, \forall v \in H_{0}^{m}\left(\Omega^{\prime}\right)\right\} ;
$$

recall that $N(\lambda):=N_{0}(\lambda ; \Omega)$ and that, by $(4.17)$,

$$
N_{0}\left(\lambda, \Omega^{\prime}\right)=N\left(\lambda ; H_{0}^{m}\left(\Omega^{\prime}\right), L^{2}(\Omega), a\right) .
$$

(See the comment following (4.34) below; of course, since $H_{0}^{m}\left(\Omega^{\prime}\right) \subset H_{0}^{m}(\Omega)$ because $\Omega^{\prime} \subset \Omega$, one could also use the more familiar Proposition 4.2(i) to derive the first inequality of (4.29).) [Observe that, with the notation of Proposition $4.3, Z_{\lambda}$ in (4.30b) is the orthogonal complement of $W_{0}:=H_{0}^{m}\left(\Omega^{\prime}\right)$ in $W:=H_{0}^{m}(\Omega)$, with respect to the inner product $a-\lambda$; this justifies, in particular, the use of the notation $\mathscr{N}(\lambda ; \omega)$ in (4.30a) since, by construction, $\Omega=\Omega^{\prime} \cup \omega$, up to a set of $|\cdot|_{n}$-measure zero. We point out, however, that the notation for 
$\mathscr{N}(\lambda ; \omega)$ is only suggestive and does not refer to a boundary value problem on $\omega$.

As was indicated in the introduction of this section, we will have to compare with $\phi(\lambda)$ the "interior term" $N_{0}\left(\lambda ; \Omega^{\prime}\right)$, as well as to estimate the "boundary term" $\mathscr{N}(\lambda ; \omega)$. In preparation for that, we note that since $\phi(\lambda)=\phi(\lambda ; \Omega)$, (4.29) implies:

$$
A_{1}+A_{2} \leq N(\lambda)-\phi(\lambda) \leq A_{1}+A_{2}+A_{3}
$$

where we have set

$$
\begin{gathered}
A_{1}=N_{0}\left(\lambda ; \Omega^{\prime}\right)-\phi\left(\lambda ; \Omega^{\prime}\right) \\
A_{2}=\phi\left(\lambda ; \Omega^{\prime}\right)-\phi(\lambda ; \Omega)
\end{gathered}
$$

and

$$
A_{3}=\mathscr{N}(\lambda ; \omega) \text {. }
$$

We first estimate $A_{1}$. In view of (4.25), Lemma 4.2 implies

$$
N_{0}\left(\lambda ; \Omega^{\prime}\right)=\sum_{q=0}^{p} \sum_{\zeta \in I_{q}} N_{0}\left(\lambda ; Q_{\zeta}^{q}\right) ;
$$

moreover, by $(4.19),(4.25)$ and since $\mu_{\mathscr{A}}^{\prime}(\cdot)$ is a measure,

$$
\phi\left(\lambda ; \Omega^{\prime}\right)=\sum_{q=0}^{p} \sum_{\zeta \in I_{q}} \phi\left(\lambda ; Q_{\zeta}^{q}\right)
$$

Hence (4.32a) and (4.33) yield

$$
\left|A_{1}\right| \leq \sum_{q=0}^{p} \sum_{\zeta \in I_{q}}\left|N_{0}\left(\lambda ; Q_{\zeta}^{q}\right)-\phi\left(\lambda ; Q_{\zeta}^{q}\right)\right| .
$$

[The shorthand notation (for the "counting function") adopted here is quite convenient for our purpose; however, some care must be exerted in interpreting it and comparing it with the more cumbersome notation of $\S 4.1$. For example, in (4.29), $W_{0}:=H_{0}^{m}\left(\Omega^{\prime}\right)$ is considered as a (closed) subspace of $W:=H_{0}^{m}(\Omega) \subset L^{2}(\Omega)$, whereas, in $(4.33 \mathrm{a})$, it is considered as a (closed) sub-

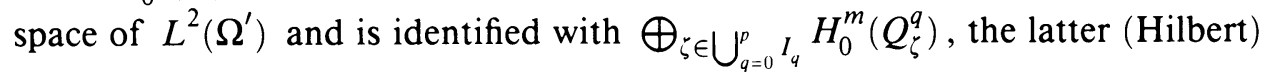
sum being orthogonal both with respect to the $L^{2}$ and $a$ inner products; see [Mt3, §V.2, especially pp. 180-182].]

We may assume that $\mathscr{A}$ is equal to its leading part $\mathscr{A}^{\prime}$; i.e., that $\mathscr{A}$ is a homogeneous operator of order $2 m$ (with locally constant coefficients). [Indeed, as will be seen in Lemma 4.4 (and Appendix B) below, the substitution of $\mathscr{A}^{\prime}$ for $\mathscr{A}$ introduces a perturbation term that does not affect the outcome.] We can thus apply Proposition 4.4(i) [with $W_{Q}=H_{0}^{m}(Q)$ ] to estimate (uniformly) 
each summand in (4.34) and deduce that there exists a positive constant $C$ such that for all $p \geq 1$ and $\lambda>0$ :

$$
\left|A_{1}\right| \leq C \sum_{q=0}^{p}\left(\# I_{q}\right)\left[1+\left(\varepsilon_{q}\right)^{n-1} \lambda^{(n-1) / 2 m}\right]=C R_{p}(\lambda)
$$

note that for every open cube $Q \subset \Omega$, the leading coefficients of $\mathscr{A}$ are constant [see Remark 4.3(b)]. Further, recall that the $I_{q}$ 's are disjoint and that for a given $q$, each cube $Q_{\zeta}^{q}$ has the same size $\varepsilon_{q}=2^{-q}$.

Next, we estimate $A_{2}$ in terms of the remaining "boundary strip" $\omega=\omega_{p}$. In view of (4.19) and (4.32b), we have successively:

$$
\begin{aligned}
\left|A_{2}\right| & =\phi(\lambda ; \Omega)-\phi\left(\lambda ; \Omega^{\prime}\right)=\left[\mu_{\mathscr{A}}^{\prime}(\Omega)-\mu_{\mathscr{A}}^{\prime}\left(\Omega^{\prime}\right)\right] \lambda^{n / 2 m} \\
& =\mu_{\mathscr{A}}^{\prime}(\omega) \lambda^{n / 2 m} \leq c^{\prime}|\omega| \lambda^{n / 2 m}
\end{aligned}
$$

note that the third equality in (4.36) follows since, by (4.24b), $\omega=\Omega \backslash \Omega^{\prime}$ up to a set of $|\cdot|$ - [and hence $\mu_{\mathscr{A}}^{\prime}(\cdot)$ ]-measure zero. [We remark that since $A_{2}=-\varphi(\lambda ; \omega) \leq 0$, we could have suppressed $A_{2}$ in the right-hand side of (4.31).] Next we recall that by $(4.26)$,

$$
\omega=\omega_{p} \subset \widetilde{\Gamma}_{c_{1} \varepsilon_{p}}
$$

further, since $\varepsilon_{p}=2^{-p} \downarrow 0$ and $\widetilde{\mathscr{M}}_{d}(\Gamma)=\lim \sup _{\rho \rightarrow 0^{+}} \rho^{-(n-d)}\left|\widetilde{\Gamma}_{\rho}\right|<+\infty$, we deduce from (4.37) as in (4.23b) that there exist $c_{1}^{\prime}$ and $p_{1}^{\prime}$ such that

$$
|\omega|=\left|\omega_{p}\right| \leq\left|\widetilde{\Gamma}_{c_{1} \varepsilon_{p}}\right| \leq c_{1}^{\prime}\left(\varepsilon_{p}\right)^{n-d} \quad \forall p \geq p_{1}^{\prime} .
$$

Equations (4.36) and (4.38) imply that for all $p \geq p_{1}^{\prime}$ and $\lambda>0$ :

$$
\left|A_{2}\right| \leq c_{1}^{\prime}\left(\varepsilon_{p}\right)^{n-d} \lambda^{n / 2 m}
$$

Finally, we estimate the "boundary term" $A_{3}:=\mathscr{N}(\lambda ; \omega)$, defined by (4.30). By [Mt3, Lemma 5.8, p. 181]-which relies, in particular, on the definition of $i$-width and Proposition 4.4(ii) - and by taking into account our notation and simpler hypotheses on the (homogeneous) form $a$, there exists a positive constant $c$ such that for all $\lambda>0$ :

$$
\mathscr{N}(\lambda ; \omega) \leq N_{0}^{*}(c \lambda ; \omega)+c R_{p}(\lambda)
$$

where $R_{p}(\lambda)$ is given by (4.28). Next, we note that for all $\lambda>0$,

$$
N_{0}^{*}(\lambda ; \omega) \leq N_{1}^{*}(\lambda ; \omega) \leq N_{1}^{*}\left(\lambda ; \widetilde{\Gamma}_{c_{1} \varepsilon_{p}}\right)
$$

Recall that for $j=0$ or $1, N_{j}^{*}(\lambda ; U)$-that occurs in (4.40) and (4.41)is defined by (4.18), and that, in view of Remark 4.4(b), $N_{j}^{*}(\lambda ; U)$ increases with $U$ and $N_{0}^{*}(\lambda ; U) \leq N_{1}^{*}(\lambda ; U)$ since $H_{0}^{m}(U) \subset \mathscr{H}_{0}^{m}(U)$; hence, the first inequality in (4.41) is immediate while the second one follows from (4.37).

We can thus apply Proposition 4.6 (from Step 1) [i.e., the "boundary estimate" for $N_{1}^{*}\left(\lambda ; \widetilde{\Gamma}_{c_{1} \varepsilon_{p}}\right)$ ] to deduce from (4.40) and (4.41) that there exist 
positive constants $\lambda_{1}>1, C_{1}^{\prime}$, and $p_{1} \geq p_{1}^{\prime}$ such that for all $p \geq p_{1}$ and $\lambda \geq \lambda_{1}\left(\varepsilon_{p}\right)^{-2 m}(\geq 1)$, we have:

$$
\mathscr{N}(\lambda ; \omega)=A_{3} \leq C_{1}^{\prime}\left(\varepsilon_{p}\right)^{n-d} \lambda^{n / 2 m}+c R_{p}(\lambda) .
$$

The conclusion of Proposition 4.7 now follows by combining (4.31), (4.32), (4.35), (4.39), and (4.42).

Remarks 4.7. (a) A great advantage of the above version of the method of "Dirichlet-Neumann bracketing" as well as of the method used to derive estimate (4.42) is that it will enable us to provide a uniform treatment of both the Dirichlet and Neumann problems, and, at the same time, to obtain sufficiently sharp estimates under minimal hypotheses.

(b) For the Neumann problem considered in $\S 4.2 . B$, the condition $\widetilde{\mathscr{M}}_{d}(\Gamma)<$ $+\infty$, used to derive (4.38), will be automatically satisfied since $\widetilde{\mathscr{M}}_{d}(\Gamma) \leq \mathscr{M}_{d}(\Gamma)$ and by assumption, $\mathscr{M}_{d}(\Gamma)<+\infty$, in that case.

In the course of the proof of Proposition 4.7, we have used the following perturbation result, which is established in Appendix B:

Lemma 4.4. If Proposition 4.7 holds for the leading part $\mathscr{A}^{\prime}$ of the operator $\mathscr{A}$, then it also holds for $\mathscr{A}$.

STEP 3. We now estimate the "remainder term" $R_{p}(\lambda)$, which as we shall see, is also of a boundary nature.

In view of (4.28), we can write for $\lambda>0$ and $p \geq 1$ :

$$
R_{p}(\lambda)=S_{p} \lambda^{(n-1) / 2 m}+T_{p}
$$

where

$$
S_{p}:=\sum_{q=0}^{p}\left(\# I_{q}\right)\left(\varepsilon_{q}\right)^{n-1} \quad \text { and } \quad T_{p}:=\sum_{q=0}^{p}\left(\# I_{q}\right) ;
$$

observe that $S_{p}$ and $T_{p}$ are independent of $\lambda$.

In the following key estimate, the roughness of the boundary $\Gamma$ will play an essential role. It will thus be convenient to introduce a coefficient which, in some sense, measures the "fractality" of $\Gamma$; set

$$
\theta=d-(n-1) \text {. }
$$

Since, by assumption, $d \in[n-1, n]$, we have $\theta \in[0,1]$. Further, if $\Gamma$ is "fractal" (i.e., $\widetilde{D} \in(n-1, n])$, then $\theta \in(0,1]$; indeed, the hypothesis that $\widetilde{\mathscr{M}}_{d}(\Gamma)<+\infty$ implies that $d \geq \widetilde{D}$, and whence $d>n-1$ in this case.

Proposition 4.8. There exist positive constants $c_{2}, c_{3}$, and $p_{2}$-depending only on $\Omega, n$, and $d$-such that the following inequalities hold for all $p \geq p_{2}$ :

(i) If $\theta \in(0,1]$, then $S_{p} \leq c_{2}+c_{3}\left(\varepsilon_{p}\right)^{-\theta}=c_{2}+c_{3} 2^{p \theta}$.

(ii) If $\theta=0$, then $S_{p} \leq c_{2}+c_{3} p$.

Here, $\theta \in[0,1]$ is defined by (4.45) and $S_{p}$ by (4.44). 
Remark 4.8. We stress that if $\Gamma$ is "fractal"-which is the case of greatest interest to us-we must necessarily be in case (i); in particular, case (ii)which corresponds to the situation studied in [Mt2,3]-can only occur if $\Gamma$ is "nonfractal".

The difference between the "fractal" and the "nonfractal" case in the remainder estimates obtained in Theorem 2.1 (and especially its corollaries) finds its origin in the dichotomy observed in the conclusion of Proposition 4.8.

Proof of Proposition 4.8. Recall that by construction [see (4.24a) and (4.26)]:

$$
\bigcup_{\zeta \in I_{q}} Q_{\zeta}^{q} \subset \omega_{q-1} \subset \widetilde{\Gamma}_{c_{1} \varepsilon_{q-1}} \quad \forall q \geq 2,
$$

with $c_{1}:=1+\sqrt{n}$. Since $\widetilde{\mathscr{M}}_{d}(\Gamma)<+\infty$, we can apply (4.23b) and deduce from (4.46) that there exist $c, c^{\prime}>0$ and $p_{2}>1$ such that $\forall q \geq p_{2}$,

$$
\left(\# I_{q}\right)\left(\varepsilon_{q}\right)^{n} \leq\left|\widetilde{\Gamma}_{c_{1} \varepsilon_{q-1}}\right| \leq c^{\prime}\left(\varepsilon_{q-1}\right)^{n-d}=c\left(\varepsilon_{q}\right)^{n-d} ;
$$

note that $\varepsilon_{q}=2^{-q}$ so that $\varepsilon_{q-1}=2 \varepsilon_{q}$.

According to (4.45) and (4.47), we have:

$$
\left(\# I_{q}\right)\left(\varepsilon_{q}\right)^{n-1} \leq c\left(\varepsilon_{q}\right)^{(n-1)-d}=c\left(\varepsilon_{q}\right)^{-\theta} \quad \forall q \geq p_{2} .
$$

Next, we fix $p>p_{2}$ and break $S_{p}$ [in (4.44)] into the two sums $\sum_{q=0}^{p_{2}}$ and $\sum_{q=p_{2}+1}^{p}$; hence we deduce that there exists a positive constant $c_{2}$ such that

$$
S_{p} \leq c_{2}+\sum_{p}
$$

where $\sum_{p}:=\sum_{q=p_{2}+1}^{p}\left(\# I_{q}\right)\left(\varepsilon_{q}\right)^{n-1}$. By (4.48), we have

$$
\sum_{p} \leq c \sum_{q=p_{2}+1}^{p}\left(\varepsilon_{q}\right)^{-\theta}
$$

Case (ii) of Proposition 4.8 follows immediately from (4.49) and (4.50) since then $\theta=0$ and hence $\sum_{p} \leq c p$.

Assume now that we are in case (i); i.e., $\theta \neq 0$. By (4.50) and since $\varepsilon_{q}=2^{-q}$, we have successively:

$$
\begin{aligned}
\sum_{p} & \leq c \sum_{q=p_{2}+1}^{p}\left(2^{\theta}\right)^{q}=c 2^{\left(p_{2}+1\right) \theta} \frac{2^{\left(p-p_{2}\right) \theta}-1}{2^{\theta}-1} \leq c 2^{\left(p_{2}+1\right) \theta} \frac{2^{\left(p-p_{2}\right) \theta}}{2^{\theta}-1} \\
& =\frac{c 2^{\theta}}{2^{\theta}-1} 2^{p \theta}=: c_{3} 2^{p \theta}=c_{3}\left(\varepsilon_{p}\right)^{-\theta} ;
\end{aligned}
$$

note that since $\theta \neq 0$, we have $2^{\theta} \neq 1$ and also $2^{\theta}-1>0$.

Consequently, case (i) follows from (4.49) and (4.51). This completes the proof of Proposition 4.8.

We conclude Step 3 by obtaining an estimate for $T_{p}$, given by (4.44). 
Lemma 4.5. There exist positive constants $c_{2}, c_{3}$, and $p_{2}$ such that the following inequalities hold for all $p \geq p_{2}$ :

(i) If $d \neq 0$, then $T_{p} \leq c_{2}+c_{3}\left(\varepsilon_{p}\right)^{-d}=c_{2}+c_{3} 2^{p d}$.

(ii) If $d=0$, then $T_{p} \leq c_{2}+c_{3} p$.

Remarks 4.9. (a) If $n \geq 2$, then we are necessarily in case (i) since $d \geq n-1>$ 0 . Moreover, case (ii) can only occur when $n=1$ and $\Gamma$ is "nonfractal" (i.e., $\widetilde{D}=n-1=0$ ). Hence-in contrast to Proposition 4.8 (and except in the one-dimensional case)-the dichotomy of Lemma 4.5 is only apparent.

(b) In agreement with one's intuition, the more irregular the boundary $\Gamma$, the larger $\theta=d-(n-1) \in[0,1]$ and $d \in[n-1, n]$, and hence the worse the estimate in case (i) of Proposition 4.8 and Lemma 4.5.

Proof of Lemma 4.5. By (4.48) [or (4.47)], there exist $c>0$ and $p_{2}>1$ such that

$$
\# I_{q} \leq c\left(\varepsilon_{q}\right)^{-d}=c 2^{q d} \quad \forall q \geq p_{2} .
$$

We now proceed as in the second part of the proof of Proposition 4.8: if $d=0$ [case (ii)], then clearly $S_{p} \leq c_{2}+c p$. On the other hand, if $d \neq 0$ [case (i)], we break $T_{p}:=\sum_{q=0}^{p}\left(\# I_{q}\right)$ into two sums and use (4.52) to conclude that:

$$
\begin{aligned}
T_{p} & \leq c_{2}+\sum_{q=p_{2}+1}^{p}\left(\# I_{q}\right) \leq c_{2}+c \sum_{q=p_{2}+1}^{p}\left(2^{d}\right)^{q} \\
& \leq c_{2}+c \frac{2^{(p+1) d}}{2^{d}-1}=: c_{2}+c_{3} 2^{p d}=c_{2}+c_{3}\left(\varepsilon_{p}\right)^{-d},
\end{aligned}
$$

as desired.

STEP 4. We now conclude the proof of Theorem 2.1. We must therefore establish estimate (4.20).

According to Proposition 4.7 (Step 2), there exist positive constants $C_{1}, p_{1}$, and $\lambda_{1}>1$ such that $\forall p \geq p_{1}$ and $\forall \lambda \geq \lambda_{1}\left(\varepsilon_{p}\right)^{-2 m}$ :

$$
|N(\lambda)-\phi(\lambda)| \leq C_{1}\left[\left(\varepsilon_{p}\right)^{n-d} \lambda^{n / 2 m}+R_{p}(\lambda)\right] .
$$

In light of Step 3, we have to distinguish two cases:

Case 1 (Case (i) of Theorem 2.1). Assume that $d \in(n-1, n]$. Then $\theta:=$ $d-(n-1) \in(0,1]$ and $d \neq 0$; hence, by case (i) of Proposition 4.8 and Lemma 4.5 (Step 3), there exist positive constants $c_{1}, c_{2}$, and $p_{2}$ such that $\forall p \geq p_{2}$ :

$$
R_{p}(\lambda) \leq\left[c_{1}+c_{2}\left(\varepsilon_{p}\right)^{-\theta}\right] \lambda^{(n-1) / 2 m}+c_{1}+c_{2}\left(\varepsilon_{p}\right)^{-d}
$$

we may as well assume that $p_{2}>p_{1}$. Combining (4.53) and (4.54), we deduce 
that $\forall p \geq p_{2}$ and $\forall \lambda \geq \lambda_{1}\left(\varepsilon_{p}\right)^{-2 m}$ :

$$
\begin{aligned}
|N(\lambda)-\phi(\lambda)| & \leq C_{1}^{\prime}\left[\left(\varepsilon_{p}\right)^{n-d} \lambda^{n / 2 m}+\lambda^{(n-1) / 2 m}+\left(\varepsilon_{p}\right)^{-\theta} \lambda^{(n-1) / 2 m}+1+\left(\varepsilon_{p}\right)^{-d}\right] \\
& =C_{1}^{\prime}\left[2^{-p(n-d)} \lambda^{n / 2 m}+\lambda^{(n-1) / 2 m}+2^{p \theta} \lambda^{(n-1) / 2 m}+1+2^{p d}\right] .
\end{aligned}
$$

Finally, we claim that if we choose $p$ such that

$$
2^{-p}=\varepsilon_{p} \approx \lambda^{-1 / 2 m} \text { and hence } 2^{p}=\left(\varepsilon_{p}\right)^{-1} \approx \lambda^{1 / 2 m} \text { as } \lambda \rightarrow+\infty,
$$

then for all $\lambda$ large enough, each term in the right-hand side of (4.55) has an upper bound of the form $C \lambda^{d / 2 m}$, as desired.

More explicitly, let $p=p(\lambda)$ be the largest integer so that $2^{p} \leq\left(\lambda / \lambda_{1}\right)^{1 / 2 m}$; i.e.,

$$
p=p(\lambda):=\left[\frac{1}{2 m} \log _{2}\left(\frac{\lambda}{\lambda_{1}}\right)\right],
$$

where $[\nu]$ denotes the integer part of $\nu$. Since, by definition, we have $2^{p} \leq$ $\left(\lambda / \lambda_{1}\right)^{1 / 2 m}$ and $2^{p+1}>\left(\lambda / \lambda_{1}\right)^{1 / 2 m}$, there exist positive constants $c_{1}^{\prime}, c_{2}^{\prime}$ so that $c_{1}^{\prime} \lambda^{1 / 2 m} \leq 2^{p}=\left(\varepsilon_{p}\right)^{-1} \leq c_{2}^{\prime} \lambda^{1 / 2 m}$; i.e., (4.56) holds. Now, let $\lambda_{2}$ be so large that $\lambda_{2}>\lambda_{1}$ and $p\left(\lambda_{2}\right) \geq p_{2}$. Then, for all $\lambda \geq \lambda_{2}$, we have with $p:=p(\lambda)$ : $\left(\varepsilon_{p}\right)^{n-d} \lambda^{n / 2 m} \leq C \lambda^{[-(n-d)+n] / 2 m}=C \lambda^{d / 2 m}, \lambda^{(n-1) / 2 m} \leq \lambda^{d / 2 m} \quad($ since $n-1 \leq d$ and $\lambda \geq 1),\left(\varepsilon_{p}\right)^{-\theta} \lambda^{(n-1) / 2 m} \leq \lambda^{[\theta+(n-1)] / 2 m}=c \hat{\lambda}^{d / 2 m}$ and $\left(\varepsilon_{p}\right)^{-d} \leq C \lambda^{d / 2 m}$. Since, by construction, $\lambda \geq \lambda_{1}\left(\varepsilon_{p}\right)^{-2 m}$ and $p:=p(\lambda) \geq p_{2}$, we thus deduce from (4.55) that

$$
|N(\lambda)-\phi(\lambda)| \leq C \lambda^{d / 2 m} \quad \forall \lambda \geq \lambda_{2} .
$$

This yields estimate (4.20) in this case and concludes the proof of case (i) of Theorem 2.1.

Remark 4.10. Let $\theta=d-(n-1)$, as before, and set $\theta^{\prime}=n-d$; then $\theta, \theta^{\prime} \in$ $[0,1]$ and $\theta+\theta^{\prime}=1$. Thus $\theta, \theta^{\prime}$-which arose naturally in the proof of Theorem 2.1-might be called the "conjugate fractional exponents" associated with the boundary $\Gamma$.

Moreover, it is easy to see that the above choice of $p$ is optimal; indeed, in view of (4.55), we must clearly have

$$
\left(\varepsilon_{p}\right)^{n-d} \lambda^{n / 2 m}=\left(\varepsilon_{p}\right)^{\theta^{\prime}} \lambda^{n / 2 m} \approx\left(\varepsilon_{p}\right)^{-\theta} \lambda^{(n-1) / 2 m}
$$

and hence $\varepsilon_{p}=\left(\varepsilon_{p}\right)^{\theta+\theta^{\prime}} \approx \lambda^{-1 / 2 m}$, as in (4.56).

Case 2 (Case (ii) of Theorem 2.1). Assume that $d=n-1$. Then $\theta=0$ and, if $n \geq 2, d \neq 0$ while if $n=1, d=0=n-1$. By case (ii) of Proposition 4.8 and by Lemma 4.5 [see Remark 4.9(a)], we have $\forall p \geq p_{2}$ :

$$
R_{p}(\lambda) \leq\left(c_{1}+c_{2} p\right) \lambda^{(n-1) / 2 m}+B
$$

where $B:=c_{1}+c_{2}\left(\varepsilon_{p}\right)^{-(n-1)}\left(\right.$ resp., $\left.B:=c_{1}+c_{2} p\right)$ if $n \geq 2$ (resp., $\left.n=1\right)$. 
Clearly, in light of (4.53) and (4.58), the optimal choice for $p$ is again given by (4.56). More precisely, we set, as in (4.57), $p=\left[(1 / 2 m) \log _{2}\left(\lambda / \lambda_{1}\right)\right]$. Then, the first term, $A$, in the right-hand side of (4.58) is the dominant one; indeed, since $\varepsilon_{p} \approx \lambda^{-1 / 2 m},\left(\varepsilon_{p}\right)^{-(n-1)} \approx \lambda^{(n-1) / 2 m}$ and thus in every case, $B \leq c A$ for all $\lambda$ large enough. Since $p \leq c^{\prime} \log \lambda$, we have $R_{p}(\lambda) \leq C^{\prime} \lambda^{(n-1) / 2 m} \log \lambda$ and, since $\left(\varepsilon_{p}\right)^{n-d} \lambda^{n / 2 m}=\varepsilon_{p} \lambda^{n / 2 m} \approx \lambda^{(n-1) / 2 m}=O\left(\lambda^{(n-1) / 2 m} \log \lambda\right)$, we conclude from (4.53) as in Case 1 that there exists $\lambda_{2}$ such that

$$
|N(\lambda)-\phi(\lambda)| \leq C \lambda^{(n-1) / 2 m} \log \lambda \quad \forall \lambda \geq \lambda_{2} .
$$

This yields estimate (4.20) in this case and concludes the proof of Theorem 2.1 for the Dirichlet problem.

Remarks 4.11. (a) Case 2, in which $d=n-1$ and $\Gamma$ is necessarily "nonfractal", corresponds to the situation studied in [Mt3, §VI.3, pp. 197-199] and-for the Laplacian and under much more restrictive assumptions on $\Omega$-in [CoHi, $\S$ VI.5, pp. 443-445]. [The (harmless) dichotomy of Lemma 4.5 does not seem to have been pointed out in [Mt2, 3].] The estimate $O\left(\lambda^{(n-1) / 2 m} \log \lambda\right)$ is the best one known for general open sets of this type.

(b) The estimate $O\left(\lambda^{d / 2 m}\right)$ obtained in Case 1 is in general the "best possible", as will be seen in $\S 5.1$. Further, as was mentioned earlier, the somewhat surprising dichotomy observed in the conclusion of Theorem 2.1 is due to that obtained in Proposition 4.8; from a technical point of view, it has its origin in the following elementary fact: the partial sums of a geometric series of ratio $2^{\theta}$ have a different form according to whether $\theta \neq 0$ or $\theta=0$. At present, we do not have a more conceptual explanation to offer although it would certainly be interesting to find one.

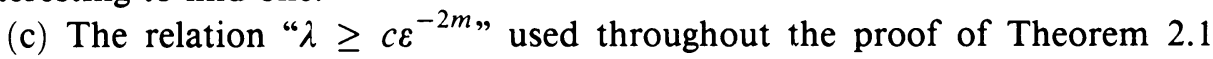
is very reminiscent of the "uncertainty principle" from quantum mechanics. [Strictly speaking, for the Laplacian $(m=1)$, for example, the relation " $\lambda \geq$ $c \varepsilon^{-2 "}$ must hold for some positive constant $c$ and all positive $\varepsilon$ small enough.] Indeed, heuristically, it can be thought of as being a precise mathematical counterpart of the following well known quantum physical fact: in order to probe the fine structure of microscopic "matter", the wavelength of the incoming "wave" must be (sufficiently) small. [One can imagine being in possession of a "fractal microscope" which at smaller and smaller scales $\varepsilon$, requires larger and larger wavenumbers $\lambda^{1 / 2}$, and hence smaller and smaller wavelengths (proportional to) $\lambda^{-1 / 2}$ ( $\leq c^{\prime} \varepsilon$, for the Laplacian). (Incidentally, this analogy helps understand why the fine details of the boundary $\Gamma$ can only be "seen" in the high energy (or equivalently, frequency) limit.)]

In a sense, our work can be viewed a posteriori as an attempt to extend "microlocal analysis"-which is traditionally valid in the "smooth" domain (see, e.g., [Ff] and references therein) - to the "fractal" realm. 
4.2.B. The Neumann problem. We explain here how to modify the proof of Theorem 2.1 in the case of Neumann boundary conditions. Towards the end of this section, we shall also give an extension of Theorem 2.1 for the Neumann problem valid, for example, for "quasidisks" and their higher-dimensional analogues.

We now assume that the hypotheses of Theorem 2.1 for Neumann (or, more generally, mixed Dirichlet-Neumann) boundary conditions are satisfied. Hence, in particular, hypotheses (2.9)-(2.11) hold and $\Omega$ is a bounded open set of $\mathbb{R}^{n}$ satisfying the " $\left(\mathrm{C}^{\prime}\right)$ condition" [hypothesis $\left.(2.13)\right]$ with boundary $\Gamma$ such that $\mathscr{M}_{d}(\Gamma)<+\infty$, for some $d \in[n-1, n]$.

In the present case, we set for $\omega$ an open subset of $\Omega$,

$$
\begin{aligned}
& N_{0}(\lambda ; \omega):=N\left(\lambda ; H_{0}^{m}(\omega), L^{2}(\Omega), a\right) \text { and } \\
& N_{1}(\lambda ; \omega)=N\left(\lambda ; H^{m}(\omega), L^{2}(\Omega), a\right) .
\end{aligned}
$$

[Note that $N_{0}(\lambda ; \omega)$ in (4.59) is defined just as in (4.17) for the Dirichlet problem.] Further, we replace $(4.18)$ by

$$
N_{1}^{*}(\lambda ; \omega):=N^{*}\left(\lambda ; H^{m}(\Omega), L^{2}(\omega)\right) .
$$

[Here, the right-hand side of (4.60) is given as in (4.8).] Since $H_{0}^{m}(\Omega) \subset V \subset$ $H^{m}(\Omega)$, by hypothesis (2.9), it follows from Proposition 4.2(i) that $N_{0}(\lambda ; \Omega) \leq$ $N(\lambda)=N\left(\lambda ; V, L^{2}(\Omega), a\right) \leq N_{1}(\lambda ; \Omega)$; consequently-and because we have established Theorem 2.1 for the Dirichlet problem in $\S 4.2$.A-it suffices to consider the Neumann problem. We thus assume from now on that $V=H^{m}(\Omega)$ and hence $N(\lambda)=N_{1}(\lambda ; \Omega)$. (See Example 4.1.)

Then, the counterpart of Proposition 4.6 for the Neumann problem still holds, but with $N_{1}^{*}\left(\lambda ; \widetilde{\Gamma}_{\varepsilon}\right)$ in (4.21) now given by (4.60) instead of (4.18). (In view of Proposition 4.5' [which can be applied since $\Omega$ satisfies the " $\left(\mathrm{C}^{\prime}\right)$ condition" by hypothesis $(2.13)$ ] and the fact that $\mathscr{M}_{d}(\Gamma)$ [and not simply $\widetilde{\mathscr{M}}_{d}(\Gamma)$ ] is assumed to be finite in this case, the proof parallels that of Proposition 4.6, as was indicated in Remark 4.6.)

The statements of the remaining results in $\S 4.2$.A stay unchanged. We now briefly indicate how to adapt the proof of Proposition 4.7 to the Neumann problem: with the present notation and according to a refinement of the method of "Dirichlet-Neumann bracketing", (4.29) [and hence (4.31)] remains valid. More precisely, we apply Proposition 4.3 , with $W_{0}:=H_{0}^{m}\left(\Omega^{\prime}\right)$, just as before, and with $W:=H^{m}(\Omega)$ [instead of $W:=H_{0}^{m}(\Omega)$ ] (as well as with $H:=L^{2}(\Omega)$ and $\left.b:=a\right)$. This yields (4.29) and hence (4.31); however, in (4.30), $\mathcal{N}(\lambda ; \omega)=N\left(\lambda ; Z_{\lambda}, L^{2}(\Omega), a\right)$, as before, but now, $Z_{\lambda}:=\{u \in$ $\left.H^{m}(\Omega): a(u, v)=\lambda(u, v)_{L^{2}(\Omega)}, \forall v \in H_{0}^{m}\left(\Omega^{\prime}\right)\right\}$ denotes the orthogonal complement of $W_{0}:=H_{0}^{m}\left(\Omega^{\prime}\right)$ in $W:=H^{m}(\Omega)$, with respect to the scalar product $a-\lambda$. Next, we note that the terms $A_{1}$ and $A_{2}$ in (4.32a) and (4.32b), respectively, are exactly the same as for the Dirichlet problem. So estimate (4.35) follows from Proposition 4.4(i) [with $W_{Q}=H_{0}^{m}(Q)$ ] . [Recall that Lemma 4.4 
is established in Appendix B both for the Dirichlet and the Neumann problems.] Further, (4.38) —and hence estimate (4.39) - still holds because if $\mathscr{M}_{d}(\Gamma)$ is finite, so is $\widetilde{\mathscr{M}}_{d}(\Gamma)$. Finally, we obtain an upper estimate for the "boundary term" $A_{3}:=\mathscr{N}(\lambda ; \omega)$, with $\omega:=\omega_{p}$. [Mt3, Lemma 5.8, p. 181] still yields the counterpart of $(4.40): \mathscr{N}(\lambda ; \omega) \leq N_{1}^{*}(c \lambda ; \omega)+c R_{p}(\lambda)$, where $N_{1}^{*}(\lambda ; U)$ is given by (4.60); further, by (4.37) and the monotonicity of $N_{1}^{*}(\lambda ; U)$ with respect to $U$ [see Remark 4.4(b)], the analogue of $(4.41)$ reads: $N_{1}^{*}(\lambda ; \omega) \leq N_{1}^{*}\left(\lambda ; \widetilde{\Gamma}_{c_{1} \varepsilon_{p}}\right)$. We then apply the counterpart of Proposition 4.6 to deduce from the last two inequalities the analogue of (4.42). We can thus conclude the proof of Proposition 4.7 as in $\S 4.2$. .A.

The proofs of Proposition 4.8, Lemma 4.5, as well as of Step 4, remain valid without change if we note-as was done earlier-that $\mathscr{M}_{d}(\Gamma)<+\infty$ implies $\widetilde{\mathscr{M}}_{d}(\Gamma)<+\infty$. This completes the proof of Theorem 2.1 for the Neumann problem.

We conclude this section by providing a generalization of Theorem 2.1 and of its corollaries in the case of Neumann boundary conditions. To do so, we shall need the following definition.

Definition 4.3. Let $V:=H^{m}(\Omega)$. An open set $\Omega$ is said to satisfy the "extension property" (for the space $V$ ) if there exists a continuous linear extension operator $\mathscr{E}: V \rightarrow H^{m}\left(\mathbb{R}^{n}\right)$ [so that every element $u$ in $V:=H^{m}(\Omega)$ is the restriction to $\Omega$ of an element $\mathscr{E} u$ in $H^{m}\left(\mathbb{R}^{n}\right)$ ].

Example 4.2. Assume that $V=H^{1}(\Omega)$ and $n=2$. Then the simply connected domain $\Omega$ satisfies the "extension property" if and only if $\Omega$ is a quasidisk (i.e., is bounded by a quasicircle $\Gamma$ ). (See [VGL] and, e.g., [M, §1.5.1].) [Recall that a quasicircle is the image of a circle under a quasiconformal map of the plane onto itself; for an equivalent characterization-due to Ahlfors-in terms of a chord-arc condition, see, e.g., [M, p. 70] and the references therein.] Such domains arise naturally, for instance, in harmonic analysis, complex analysis and approximation theory. For a concrete example, the reader may wish to consult [M, Example 1, pp. 70-71].

It is noteworthy that the boundary $\Gamma$ can then be arbitrarily irregular; indeed, it is possible to construct-as is done in [GeVa] - a family of quasidisks for which $H$, the Hausdorff dimension of $\Gamma$, takes on every value in $[1,2)$.

More generally, for $V=H^{m}(\Omega)$ and $n \geq 2$, sufficient conditions for the domain $\Omega$ to satisfy the "extension property" (for $V$ ) are obtained by Jones in [Jn]. This class of "Jones domains" contains that of strong Lipschitz domains (see, e.g., [M, §1.1.16]) and, when $n=2$, coincides with that of quasidisks.

We are now in a position to state the promised generalization of Theorem 2.1:

Theorem 4.1. Let $\Omega$ be a bounded open subset of $\mathbb{R}^{n}$. Then, for the Neumann problem, all the results of $\S 2.2$ (namely, Theorems 2.1-2.3 as well as Corollary 
2.2) still hold if in hypothesis (2.13), we replace the " $\left(\mathrm{C}^{\prime}\right)$ condition" by the "extension property" [ for the space $\left.V:=H^{m}(\Omega)\right]$.

More generally, the present theorem extends to the Dirichlet-Neumann problem if in Definition 4.3, we replace the space $H^{m}(\Omega)$ by $V$, with $V$ as in hypothesis (2.9).

Proof. This is established in the same way as Theorem 2.1 for the Neumann problem if we observe that Proposition $4.5^{\prime}$ (and hence the counterpart of Proposition 4.6) remains valid if $\Omega$ obeys the "extension property" instead of the " $\left(\mathrm{C}^{\prime}\right)$ condition", a consequence of [BiSo, Ek] and the method of proof of Proposition 4.5.

Remarks 4.12. (a) When $\Gamma$ is "nonfractal", a special case of Theorem 4.1 (or, more precisely, of the counterpart of Theorem 2.1 and Corollary 2.2) is obtained in $[\mathrm{Mt} 2]$.

(b) I wish to thank Professors Alexander G. Ramm and Stephen William Semmes for pointing out references [M] and [Jn], respectively, after they heard me lecture on these results.

(c) Of course, in Theorem 4.1, the Neumann spectrum is discrete.

\section{EXAMPLES, CONJECTURE AND OPEN PROBLEMS}

We now illustrate our results by considering various concrete examples $(\S 5.1)$; this will show, in particular that our remainder estimates are in general "best possible". We also propose several open problems and a conjecture; the latterstated in $\$ 5.2$-extends and modifies the Weyl-Berry conjecture.

5.1. Examples. Let $\Omega$ be a bounded open set of $\mathbb{R}^{n}(n \geq 1)$, with boundary $\Gamma$; as before, we denote by $D=D(\Gamma)$ or $H=H(\Gamma)$ the Minkowski or Hausdorff dimension of $\Gamma$, respectively; further, $\widetilde{D}=\widetilde{D}(\Gamma)$ stands for the Minkowski dimension of $\Gamma$, relative to $\Omega$. (See Definitions 2.1 and 3.1.)

Unless otherwise specified, we shall always work with the Dirichlet Laplacian in the following examples. (Recall that since we deal with the Dirichlet problem, $\Omega$ is allowed to be an arbitrary bounded open set; of course, our results could also apply, under appropriate assumptions, to higher order operators as well as Neumann boundary conditions.) It will be convenient to set $\phi(\lambda)=(2 \pi)^{-n} \mathscr{B}_{n}|\Omega|_{n} \lambda^{n / 2}$, with $\mathscr{B}_{n}=\pi^{n / 2} /(n / 2) !$, the volume of the unit ball in $\mathbb{R}^{n}$, as before. (Hence, $\phi(\lambda)=\pi^{-1}|\Omega|_{1} \lambda^{1 / 2}$ or $(4 \pi)^{-1}|\Omega|_{2} \lambda$, for $n=1$ or 2 , respectively.)

Our first example will show in particular that our remainder estimates are "best possible" and that the original Berry conjecture-expressed in terms of the Hausdorff instead of the Minkowski dimensions-fails in general. It will also provide us with further information about the relationships between "fractal" and spectral geometry. Moreover, Example 5.1 will easily be generalized to yield a one-parameter family of examples in $\mathbb{R}^{n} \quad(n \geq 1)$ having similar features and for which the Minkowski dimension $D$ (equal to $\widetilde{D}$ in this case) takes on every 
value in $(n-1, n)$ whereas $H \equiv n-1$. (See Example 5.1'.)

Example 5.1. Fix an arbitrary positive real number $a$. Consider the following bounded open set $\Omega \subset \mathbb{R}$ :

$$
\Omega=\bigcup_{i=1}^{\infty}\left((i+1)^{-a}, i^{-a}\right) ;
$$

then

$$
\Gamma=\bar{A}=A \cup\{0\}, \quad \text { where } A:=\left\{i^{-a}: i=1,2, \ldots\right\} .
$$

Further, we claim that

$$
H=0, \quad \widetilde{D}=D=(a+1)^{-1}, \quad \text { and } 0<\widetilde{\mathscr{M}}_{\widetilde{D}}(\Gamma)<+\infty ;
$$

moreover, a direct computation yields

$$
\phi(\lambda)-N(\lambda) \approx \lambda^{\widetilde{D} / 2} \text { as } \lambda \rightarrow+\infty .
$$

[Recall that this means that there exist (strictly) positive constants $c_{1}, c_{2}$, and $\lambda_{0}$ such that $\left.c_{1} \lambda^{\widetilde{D} / 2} \leq \phi(\lambda)-N(\lambda) \leq c_{2} \lambda^{\widetilde{D} / 2}, \forall \lambda \geq \lambda_{0}.\right]$ Here, $\phi(\lambda)=\pi^{-1} \lambda^{1 / 2}$ since $n=1$ and $|\Omega|_{1}=1$, and $\lambda^{\widetilde{D} / 2}=\lambda^{1 / 2(a+1)}$, by (5.2).

According to Theorem 2.3 (or, more precisely, the analogue of Corollary 2.1 for the Dirichlet Laplacian) and since $\widetilde{\mathscr{M}}_{\widetilde{D}}(\Gamma)<+\infty$ by $(5.2)$, we know (without any calculation) that

$$
|\phi(\lambda)-N(\lambda)|=O\left(\lambda^{\widetilde{D} / 2}\right) \text { as } \lambda \rightarrow+\infty .
$$

In view of (5.3), we conclude that our remainder estimate (5.4) [which also clearly follows from (5.3) in this situation] is sharp in this case. Moreover, since by (5.2), $H=0$, Berry's conjecture-as formulated in [Be1, 2] in terms of the Hausdorff dimension-obviously fails here.

We now briefly justify (5.2). (See Appendix $C$ for a complete derivation.) Since $\Gamma$ is countable, $H=H(\Gamma)=0$. (See $\S 3$.) Further, we have proved in Example 3.2 that $D(A)=(a+1)^{-1}$; thus $D=D(\Gamma)=D(\bar{A})=D(A)=(a+1)^{-1}$. Moreover, a direct calculation-based on (2.6) of Definition 2.1-shows that $0<\widetilde{\mathscr{M}}_{D}(\Gamma)<+\infty$. [Actually, we can show that

$$
\widetilde{\mathscr{M}}_{D}(\Gamma)=2^{a /(a+1)}\left(a^{1 /(a+1)}+a^{-a /(a+1)}\right)=a^{D}\left(2^{1-D} /(1-D)\right) ;
$$

the interested reader will find a proof of this fact in Appendix C.] In view of Remark 2.1(b), this implies in particular that $\widetilde{D}=D=(a+1)^{-1}$ and thus yields (5.2).

Remarks 5.1. (a) Actually, if we assume that estimate (5.3) has already been established, with $\widetilde{D}$ replaced by $D=(a+1)^{-1}$, there is another instructive method for showing that $\widetilde{D}=D$; it can also be used in similar situations. In view of Remark $2.1(\mathrm{a})$, we always have $\widetilde{D} \leq D$. Moreover, if $\widetilde{D}<D$, then we 
can choose $d \in(\widetilde{D}, D)$; for this $d,|\phi(\lambda)-N(\lambda)|=O\left(\lambda^{d / 2}\right)$, by Theorem 2.3, since $d>\widetilde{D}$. (Observe that we do not use here the fact that $\widetilde{\mathscr{M}}_{\widetilde{D}}(\Gamma)<+\infty$.) On the other hand, $|\phi(\lambda)-N(\lambda)| \approx \lambda^{D / 2}$, by assumption; hence a contradiction since $d<D$. We thus conclude that $\widetilde{D}=D$.

(b) We note that the one-dimensional example associated with a given value of $a$ could arise "physically" by studying the steady-states vibrations of a string with infinitely many nodes located at the points 0 and $i^{-a}(i=1,2, \ldots)$.

We now indicate how to establish estimate (5.3). Recall that the eigenvalues of $-d^{2} / d x^{2}$ on the open interval $I:=(\alpha, \beta)$, with Dirichlet boundary conditions at $\alpha$ and $\beta$, are $\lambda_{j}=(\pi / l)^{2} j^{2}$, for $j=1,2, \ldots$, where $l:=\beta-\alpha$. If $N(\lambda ; I)$ denotes the associated "counting function", and if $[\nu]$ denotes the integer part of $\nu$, it follows in particular that $N(\lambda ; I)=0$ for $\lambda<\pi^{2} / l^{2}$ and

$$
N(\lambda ; I)=\#\{j \geq 1: j \leq l(\sqrt{\lambda} / \pi)\}=[l(\sqrt{\lambda} / \pi)] \text { for } \lambda>0 .
$$

Set $I_{i}=\left((i+1)^{-a}, i^{-a}\right)$ and $l_{i}=i^{-a}-(i+1)^{-a} \approx i^{-(a+1)}$, as $i \rightarrow \infty$. Let

$$
i(\lambda)=\max \left\{i \geq 1: l_{i} \geq \pi / \sqrt{\lambda}\right\} ;
$$

clearly, $i(\lambda) \approx(\pi / \sqrt{\lambda})^{-1 /(a+1)} \approx \lambda^{1 / 2(a+1)}=\lambda^{D / 2}$, as $\lambda \rightarrow+\infty$, since $D=$ $1 /(a+1)$. Furthermore, Lemma 4.2 and (5.5) yield

$$
N(\lambda)=\sum_{i=1}^{i(\lambda)} N\left(\lambda ; I_{i}\right)=\sum_{i=1}^{i(\lambda)}\left[l_{i}\left(\frac{\sqrt{\lambda}}{\pi}\right)\right] .
$$

Consequently, since $\phi(\lambda)=\sqrt{\lambda} / \pi$ and $1-\sum_{i=1}^{i(\lambda)} l_{i}=(i(\lambda)+1)^{-a} \approx \lambda^{-(1-D) / 2}$, as $\lambda \rightarrow+\infty$, because $a D=a /(a+1)=1-D$, it easily follows that

$$
\begin{aligned}
\phi(\lambda)-N(\lambda) & =\frac{\sqrt{\lambda}}{\pi}-\sum_{i=1}^{i(\lambda)}\left[l_{i}\left(\frac{\sqrt{\lambda}}{\pi}\right)\right] \approx \frac{\sqrt{\lambda}}{\pi}\left(1-\sum_{i=1}^{i(\lambda)} l_{i}\right) \\
& =\frac{\sqrt{\lambda}}{\pi}(i(\lambda)+1)^{-a} \approx \lambda^{(1 / 2)-((1-D) / 2)}=\lambda^{D / 2},
\end{aligned}
$$

as $\lambda \rightarrow+\infty$; hence (5.3) holds. [In order to justify the first sign " $\approx$ " in (5.8), one can use simple inequalities comparing the integer and "real" parts, as well as the aforementioned asymptotic behavior of $i(\lambda)$. More precisely, let $f(\lambda)$ [resp., $g(\lambda)$ ] denote the second (resp., third) term in (5.8); then clearly, $f(\lambda)=$ $g(\lambda)+\sum_{i=1}^{i(\lambda)}\left\{l_{i}(\sqrt{\lambda} / \pi)\right\}$ (where $\{\nu\}:=\nu-[\nu] \in[0,1)$ denotes the fractional part of $\nu$ ) and hence $g(\lambda) \leq f(\lambda) \leq g(\lambda)+i(\lambda)$ or, equivalently, $1 \leq f(\lambda) / g(\lambda) \leq$ $1+(i(\lambda) / g(\lambda))$; from which it follows that $f(\lambda) \approx g(\lambda)$ since by the second equality in (5.8), $i(\lambda) / g(\lambda) \approx \lambda^{D / 2} / \lambda^{D / 2}=1$, as $\lambda \rightarrow+\infty$.]

We have thus obtained a one-parameter family of (one-dimensional) examples for which our remainder estimate is sharp and Berry's original conjecture fails; indeed, as $a$ varies in $(0,+\infty), H \equiv 0$ whereas $\widetilde{D}$ takes on every value in $(0,1)$. 
Remarks 5.2. (a) This simple example illustrates very clearly some of the basic differences between the Minkowski and Hausdorff dimensions mentioned in $\S 3$. Indeed, the fact that $H=H(\Gamma)=0$ because $\Gamma$ is countable follows since Hausdorff measure is induced by an outer measure and is, in particular, countably subadditive. On the other hand, the fact that the Minkowski content is only finitely subadditive and is thus a premeasure (or "content") but does not induce a measure, enables one to have $D=D(\Gamma) \neq 0$ in this case. Consequently, the "bad" theoretical properties of the Minkowski dimension in this context are crucial for obtaining our results as well as for investigating the spectral properties of "fractals".

(b) One might hope to replace the Minkowski dimension by a different type of fractional dimension having "better" theoretical properties. In particular, the notion of monotone " $\sigma$-stable index" (or dimension) comes to mind. (The set function $\rho: \mathscr{P}\left(\mathbb{R}^{n}\right) \rightarrow(0,+\infty)$ is said to be " $\sigma$-stable" if $\rho\left(\bigcup_{i=1}^{\infty} A_{i}\right)=$ $\sup _{i>1} \rho\left(A_{i}\right)$; see [Tr2, pp. 58-59]. Here, $\mathscr{P}\left(\mathbb{R}^{n}\right)$ denotes the power set of $\mathbb{R}^{n}$.) Besides the Hausdorff dimension, a special case of $\sigma$-stable index is the "packing dimension", Dim, recently introduced in [Tr2], and which is a sort of regularization of the Minkowski dimension; see also the review paper by $\mathrm{J}$. Peyrière in [Ce, pp. 151-157]. [By [ $\operatorname{Tr} 2$, Corollary 1, p. 60], we have $H(A) \leq \operatorname{Dim}(A) \leq D(A)$, for any $A \subset \mathbb{R}^{n}$, and hence $\operatorname{Dim}$ would have been a natural candidate for trying to improve our remainder estimates and possibly modify Berry's original conjecture accordingly.] However, once again, the above example rules out this class of "fractal" dimensions since, in particular, the $\sigma$-stability implies that the dimension of a countable set-like that of a single point-must be equal to zerc.

These considerations reinforce us in the belief that the modified Weyl-Berry conjecture-as stated in $\S 5.2$ below-is properly formulated (especially for the Dirichlet problem).

(c) In the process of checking that $0<\widetilde{\mathscr{M}}_{\widetilde{D}}(\Gamma)<+\infty$, one shows that $\widetilde{\mathscr{M}}_{\widetilde{D}}(\Gamma)$ is the limit (and not just the upper limit) of $\varepsilon^{-(n-\widetilde{D})}\left|\widetilde{\Gamma}_{\varepsilon}\right|$, as $\varepsilon \rightarrow 0^{+}$. [See Theorem $\mathrm{C}$ (as well as Remarks $\mathrm{C}$ ) in Appendix C.] It follows that $\Gamma$ is Minkowski measurable, relative to $\Omega$ (in the sense of Definition 2.1). Hence, this simple example is a good testing ground for verifying the (modified) Weyl-Berry conjecture. (See Remark 5.7(d).)

(d) Identical calculations show that for Neumann boundary conditions and/or when $\mathscr{A}:=(-\Delta)^{m}$ (with $m \geq 1$ ), our remainder estimates are also sharp in the counterpart of Example 5.1. [Note that our hypotheses for the Neumann problem are satisfied since, for instance, $\Omega$ clearly satisfies the "extension property" for $H^{m}(\Omega)$ (see Definition 4.3 and Theorem 4.1).] (Comments entirely similar to (c) and (d) apply to Example $5.1^{\prime}$ below except that in (d), for the Neumann problem, one must substitute " $N(\lambda)-\phi(\lambda)$ " for " $\phi(\lambda)-N(\lambda)$ " in $(5.3)$ and its proof, when $n \geq 2$.)

Example 5.1' . The previous example extends naturally to higher dimensions. 
In fact, set $\Omega=\bigcup_{i=1}^{\infty}\left(I_{i} \times J\right)$, where $J:=(0,1)^{n-1}$ and $n \geq 1$. (When $n=1$, $\Omega$ is the same open set as in Example 5.1.) Then $\Omega \subset \mathbb{R}^{n}$ and analogous statements hold in this case. In particular, $\widetilde{D}=D=(n-1)+(a+1)^{-1}$ and hence, as the parameter $a$ runs through $(0,+\infty), \widetilde{D}$ takes on every value in $(n-1, n)$ whereas $H \equiv n-1$; further, $0<\widetilde{\mathscr{M}}_{\widetilde{D}}(\Gamma)<+\infty$. [More precisely, we can show that $\widetilde{\mathscr{M}}_{\widetilde{D}}(\Gamma)=\left(2^{1-\widetilde{D}} /(1-\widetilde{D})\right) a^{\widetilde{D}}$ and that $\Gamma$ is even Minkowski measurable.] (See Remarks $C$ in Appendix C.)

In this manner, we obtain a one-parameter family of examples for which our remainder estimate $(5.4)$ is "best possible", in the sense that estimate $(5.3)$ holds; in addition, for any of these examples, Berry's original conjecture clearly fails whereas our "modified Weyl-Berry conjecture"-stated in $\S 5.2$ below-is quite likely to hold.

Remarks 5.3. (a) Just as in Example 5.1, the "conjugate fractional exponents" of $\Gamma$-as defined in Remark 4.10 by $\theta=\widetilde{D}-(n-1)=\{\widetilde{D}\}$ and $\theta^{\prime}=n-\widetilde{D}=$ $1-\theta$-are equal to $(a+1)^{-1}$ and $a(a+1)^{-1}$, respectively. (Since $\widetilde{D} \in[n-1, n]$, $\theta$ (resp., $\theta^{\prime}$ ) could be called the fractional dimension (resp., codimension) of $\Gamma$.) Clearly, the symmetry $a \leftrightarrow a^{-1}$ exchanges $\theta$ and $\theta^{\prime}$; it is therefore natural to call the case when $\theta\left(=\theta^{\prime}\right)=1 / 2$ - which corresponds to the value $a=1$ of the parameter-the "midfractal" case. (Recall that $\theta+\theta^{\prime}=1$, with $\theta, \theta^{\prime} \in[0,1]$.) Finally, we note that the smaller $a$, the larger $\theta$ and $\widetilde{D}$, and hence the more "fractal" the boundary $\Gamma$.

(b) It would be interesting to slightly modify Example $5.1^{\prime}$ in order to obtain a connected (planar) open set $\Omega$ (a "comb" with finer and finer teeth, for instance) for which the counterpart of both (5.2) and (5.3) can be established.

Example 5.2. We now consider the main example in [BrCa]. Let $\Omega \subset \mathbb{R}^{2}$ be the (disconnected) bounded open set studied in [BrCa, Example 2, pp. 107-112]; $\Omega$ is the countable disjoint union of all the small open cubes belonging to the successive "generations" defined below.

Let $\left\{P_{i}\right\}_{i=1}^{\infty}$ be a nondecreasing sequence of positive integers. The 0 th generation contains one square of side 1 , considered as being both small and large; the 1st generation contains four large squares, each of which has side $1 / 3$ and is divided into $\left(P_{1}\right)^{2}$ congruent small squares, etc. Similarly, the $i$ th generation is composed of $4 \times 5^{i-1}$ large squares, each of which has side $3^{-i}$ and is divided into $\left(P_{i}\right)^{2}$ congruent small squares; and so on. (See [BrCa, Figure 1, p. 107].)

It is shown in $\left[\mathrm{BrCa}\right.$, p. 108] that, irrespective of the sequence $\left\{P_{i}\right\}, H=$ $\log 5 / \log 3$ and $0<\mathscr{H}_{H}(\Gamma)<+\infty$. Now, given any fixed real number $a \geq 1$, we let $P^{i}=\left[a^{i}\right]$ for $i=1,2, \ldots$, in order to simplify the calculations. Then, according to [ $\mathrm{BrCa},(2.2)$ and (2.5), pp. 110 and 112], we have

$$
\widetilde{D}=\log \left(5 a^{2}\right) / \log (3 a) \text { and } 0<\widetilde{\mathscr{M}}_{\widetilde{D}}(\Gamma)<+\infty
$$


note that $H=\widetilde{D}$ if and only if $a=1$ and that $H$ remains fixed whereas $\widetilde{D}$ takes on every value in $[H, 2)$, as $a$ varies in $[1,+\infty)$. Moreover, by $[\mathrm{BrCa}$, (2.1), p. 110], estimate (5.3) holds with $\widetilde{D}$ given by (5.9); here, $\phi(\lambda)=(2 \pi)^{-1} \lambda$ since $|\Omega|_{2}=1+\frac{4}{5} \sum_{i=1}^{\infty}\left(\frac{5}{9}\right)^{i}=2$. [Recall that we work here with $\mathscr{A}=-\Delta$ and not with $\mathscr{A}=-\frac{1}{2} \Delta$, as in [BrCa]; see Remark 2.5(b).] Estimate (5.3) is obtained by explicit computation.

Further, since $\widetilde{\mathscr{M}}_{\widetilde{D}}(\Gamma)<+\infty$ by $(5.9)$, Theorem 2.3 yields (without any calculation) estimate (5.4), with $\widetilde{D}$ as above. In view of (the counterpart of) (5.3) and (5.4), our remainder estimate is also sharp in this case. In addition, as was observed in [BrCa], Berry's original conjecture fails when $a>1$ since then $H<\widetilde{D}$ and (5.3) holds.

Let $Z(t)=\int_{0}^{\infty} e^{-\lambda t} d N(\lambda)$ be the "partition function" associated with the present problem. Then the pre-Tauberian form of (5.3) is

$$
(2 \pi t)^{-1}-Z(t) \approx t^{-\widetilde{D} / 2} \text { as } t \rightarrow 0^{+}
$$

this is deduced from (5.3) by means of an Abelian argument. (See the method of proof of Theorem A in Appendix A.) Moreover, Theorem 2.2 yields the counterpart of $(5.4)$ :

$$
\left|(2 \pi t)^{-1}-Z(t)\right|=O\left(t^{-\widetilde{D} / 2}\right) \quad \text { as } t \rightarrow 0^{+} .
$$

Hence, in light of (5.10) and (5.11), our remainder estimate for the "partition function" is also sharp in this case. [Of course, an entirely analogous comment could be made about Examples 5.1 and 5.1 ${ }^{\prime}$ above.]

Remarks 5.4. (a) In contrast to Examples 5.1 and $5.1^{\prime}$, it is not known whether $\Gamma$ is Minkowski measurable, relative to $\Omega$, in this case. (See [BrCa, p. 112].)

(b) Even for this rather simple example, our results provide some additional information. Indeed, when $\left\{P_{i}\right\}$ is an arbitrary nondecreasing sequence, it is not possible in general to compute $\widetilde{D}$ explicitly or to establish (5.3) [or even (5.4)] by a direct calculation. Nevertheless, Theorem 2.3 still yields (5.4), except for $\widetilde{D}$ replaced by $d>\widetilde{D}$, with $d$ arbitrarily close to $\widetilde{D}$.

(c) It is clear that $H$-which, as was recalled earlier, is independent of $\left\{P_{i}\right\}-$ cannot take into account the fine scale structure of $\Gamma$ as well as $\widetilde{D}$ does; intuitively, as was stressed in $\S 3$, this is due to the fact that the Hausdorff (resp., Minkowski) dimension is defined in terms of cubes of size $\leq \varepsilon$ (resp., $=\varepsilon)$, with $\varepsilon$ arbitrarily small. (See, in particular, Corollary 3.1.)

In [ $\mathrm{BrCa}$, pp. 112-113], it is shown by means of a probabilistic argument how a slight change of $\Omega$ yields a connected open set $\Omega^{\prime}$, with boundary $\Gamma^{\prime}$, 
for which $\left(\left|\Omega^{\prime}\right|_{2}=|\Omega|_{2}=2\right.$ and $)$

$$
(2 \pi t)^{-1}-Z^{\prime}(t) \approx t^{-\widetilde{D} / 2} \text { as } t \rightarrow 0^{+}
$$

however, it is mentioned there that the authors could not control the asymptotics of $N^{\prime}(\lambda)$ in the process. (Here, $N^{\prime}(\lambda)$ [resp., $Z^{\prime}(t)$ ] denotes the "counting [resp., partition] function" associated with the Dirichlet Laplacian on $\Omega^{\prime}$. Further, $\Omega^{\prime}$ is obtained by slightly opening up the sides of the large squares in each generation involved in the definition of $\Omega$; see [ $\mathrm{BrCa}$, Figure $4, \mathrm{p} .112]$.)

We now indicate how to use our results to obtain further information about this (modified) example. By construction, $\Gamma^{\prime} \subset \Gamma$ and $\Omega \subset \Omega^{\prime}$; hence, by a simple extension of Remark $2.1(\mathrm{e})$ and with the obvious notation, $\widetilde{D}^{\prime}:=$ $D\left(\Gamma^{\prime} ; \Omega^{\prime}\right) \leq D(\Gamma ; \Omega)=: \widetilde{D}$. Next, we claim that $\widetilde{D}=\widetilde{D}^{\prime}$. To see this, we argue much as in Remark 5.1(a): assume that $\widetilde{D}^{\prime}<\widetilde{D}$ and then choose $d \in\left(\widetilde{D}^{\prime}, \widetilde{D}\right)$; for this $d$, Theorem 2.3 (or 2.2) yields, since $d>\widetilde{D}^{\prime}:\left|(2 \pi t)^{-1}-Z^{\prime}(t)\right|=$ $O\left(t^{-d / 2}\right)$, as $t \rightarrow 0^{+}$. Since $d<\widetilde{D}$, this contradicts $(5.12)$. We thus conclude that $\widetilde{D}=\widetilde{D}^{\prime}$.

Now that we know that $\widetilde{D}=\widetilde{D}^{\prime}$, we can easily check that $\widetilde{\mathscr{M}_{\widetilde{D}}}\left(\Gamma^{\prime}\right)$ is finite; indeed, since $\Gamma^{\prime} \subset \Gamma$ and by $(5.9)$, we have $\widetilde{\mathscr{M}}_{\widetilde{D}}\left(\Gamma^{\prime}\right) \leq \widetilde{\mathscr{M}}_{\widetilde{D}}(\Gamma)<+\infty$.

Consequently, this enables us to apply Theorems 2.3 and 2.2 to deduce that

$$
\left|\phi(\lambda)-N^{\prime}(\lambda)\right|=O\left(\lambda^{\widetilde{D}^{\prime} / 2}\right) \text { and }\left|(2 \pi t)^{-1}-Z^{\prime}(t)\right|=O\left(t^{-\widetilde{D}^{\prime} / 2}\right),
$$

as $\lambda \rightarrow+\infty$ and $t \rightarrow 0^{+}$, respectively. In view of (5.12) and (5.13) and since $\widetilde{D}^{\prime}=\widetilde{D}$, this provides us with an example of a connected open set for which our remainder estimate for the "partition function" is sharp. It is very likely-although not proved here-that our remainder estimate for the "counting function" is also sharp in this case.

Example 5.3 (Planar spirals). Given the importance of spirals for the description of patterns occurring in Nature (see, e.g., [Th, especially Chapter VI]), the present example-which is inspired by [DMT] and supplements Example 3.4-may be of interest in later applications.

Let $\Omega \subset \mathbb{R}^{2}$ be a relatively compact domain bounded by the planar spiral $A$. Here, $A$ is assumed to be bounded and to rotate around the origin while converging to it. More explicitly, let $f$ be a decreasing continuous function on $[0,+\infty)$ such that $f(\theta) \rightarrow 0$, as $\theta \rightarrow+\infty$; then, the polar equation of the spiral $A$ is given by $r=f(\theta)$.

Strictly speaking, the boundary $\Gamma=\partial \Omega$ is the disjoint union of the graph of the spiral $A$, the origin $O$ and the (open) horizontal line segment $L$ connecting the points $(f(0), 0)$ and $(f(2 \pi), 0)$. However, this does not affect our discussion because $\Gamma=\bar{A} \cup L$, with $\bar{A}=A \cup\{O\}$; whence

$$
D=D(\Gamma)=\max (D(A), D(\{O\}), D(L))=D(A)
$$

and

$$
H=H(\Gamma)=\max (H(A), H(\{O\}), H(L))=H(A),
$$


since $H(\{O\})=D(\{O\})=0, D(L)=H(L)=1$ while $D(A) \geq H(A) \geq 1$.

Now, it follows from Example 3.4 that $H=1$, the topological dimension of $\Gamma$, whereas $D$ can take any value in $[1,2]$. Intuitively, the slower the convergence to the origin, the larger $D$; that is, the more "fractal" $\Gamma$. [The results about the dimensions of spirals used in this example are derived in [DMT].]

Assume that $f(\theta)=(1+\theta)^{-a}$, with $a>0$; then $D=\max \left(1,2(1+a)^{-1}\right)$ and $0<\mathscr{M}_{D}(\Gamma)<+\infty$. Hence, if $a<1$ (resp., $a \geq 1$ ), Theorem 2.3 yields (since $D \geq \widetilde{D}$ )

$$
|N(\lambda)-\phi(\lambda)|=O\left(\lambda^{D / 2}\right) \quad\left[\text { resp., } O\left(\lambda^{D / 2} \log \lambda\right)\right] \quad \text { as } \lambda \rightarrow+\infty,
$$

where $\phi(\lambda)=(2 \pi)^{-1}|\Omega|_{2} \lambda$ and $D=2(1+a)^{-1} \in(1,2)$ [resp., $D=1$ ] .

Remarks 5.5. (a) We do not know whether the remainder estimate (5.14) is sharp although we suspect that this is so, at least in the "fractal" case when $D>1$.

(b) If $f(\theta)=\exp (-\theta)$, then $D=1$; on the other hand, if $f(\theta)=$ $(\log (2+\theta))^{-1}$, then $D=2$ since $\Gamma$ tends to fill in a neighborhood of the origin, a situation reminiscent of the Peano curve. Of course, in the latter most extreme case-as was pointed out in Remark $2.4(\mathrm{~d})-(5.14)$ cannot be sharp since $\lambda=\lambda^{D / 2}$ is not $o(\lambda)=o\left(\lambda^{n / 2}\right)$ whereas Weyl's asymptotic formula (2.17) holds.

Example 5.4 (Koch drum). In our examples thus far, the boundary $\Gamma$ was not assumed to be "self-similar". We now consider the case when $\Gamma$ is one of the classical "self-similar fractals". It should naturally play an important role both as a mathematical and physical model.

Let $\Gamma$ be the triadic Koch curve, also called "snowflake curve" in the literature. (See, e.g., [Kh and Md1, pp. 42-45].) It follows from the self-similarity of $\Gamma$ [see especially Remarks 3.4. and Lemma 3.1(i)] that $D=H=\log 4 / \log 3=$ $1.2618 \ldots$ and $\mathscr{M}_{D}(\Gamma)<+\infty$. (This latter fact can also be checked directly.) Let $\Omega \subset \mathbb{R}^{2}$ be the bounded domain having for boundary $\Gamma$. Then, according to Theorems 2.3 and 2.2, $|N(\lambda)-\phi(\lambda)|=O\left(\lambda^{D / 2}\right)$, as $\lambda \rightarrow+\infty$; and similarly for the "partition function" $Z(t)$. This example has many significant physical applications, including, in particular, to the study of the vibrations of a "Koch drum", the scattering of radio-waves by a "triadic Koch island" [Be 1, 2], as well as to the propagation of waves at the surface of a lake surrounded by a "triadic Koch coastline" [Md1, pp. 40-45].

An interesting-albeit difficult-open problem is to determine whether "one can hear the fractal dimension of a Koch drum"; that is, whether the above remainder estimate for $N(\lambda)$ is best possible or, more ambitiously, whether the modified Weyl-Berry conjecture-as formulated in $\S 5.2$ below-holds in this case. [We conjecture that our error estimates are sharp but that there is no second term proportional to $\lambda^{D / 2}$, in this situation. Indeed, the hypotheses of Conjecture 5.1' below are satisfied, but not those of Conjecture 5.1.] 
Remarks 5.6. (a) Our present results are well out of reach of the methods of classical spectral geometry (e.g., [C, GuKz, Iv1,2, Ms1,2, OsWi, Ph, Se1,2], and relevant references therein) since the boundary $\Gamma$-considered as a parametrized curve-is nonrectifiable and nowhere differentiable.

(b) Naturally, we could replace $\Gamma$ by a more general "von Koch-Mandelbrot" curve of the kind considered in [Md1, Chapter 6 or Ce, $\S 1 . b$ and p. 30]; it is noteworthy that if such a (closed) curve is simple and self-similar, then $D=$ $H=\log N / \log (1 / r)$, as in Lemma 3.1(i), and hence $D$ takes on every value in [1, 2], as one varies the parameters $N$ and $r$. (See, e.g., [Md1, p. 39].)

(c) We could also consider three-dimensional analogues of Example 5.4: for instance [Be1, 2], a "fractal resonator"; that is, in our case, a resonant cavity with "fractal" boundary.

Example 5.5 (Cantor graph, combs, and quasidisks). Finally, we mention some other examples to which our results can be applied. For the Neumann Laplacian, our remainder estimates hold, for instance, in the following situations: open sets with cusp, bounded, in particular, by the graph of the Cantor singular function (recall that Theorem 2.3 can be applied in this case since the " $\left(C^{\prime}\right)$ condition" is satisfied; see Example 2.2); further, domains bounded by quasicircles (quasidisks) and their higher-dimensional analogues (Jones domains [Jn]); recall that Theorem 4.1 applies here because the "extension property" holds for $H^{1}(\Omega)$ ). (See Example 4.2.) A special case of interest is provided by the quasidisk described in [M, Example 1, pp. 70-71]. A related and physically significant example to which our results for the Neumann problem apply is provided by the "Koch drum" studied in Example 5.4, since it can be shown as in [M, pp. 70-71] that the snowflake curve is a quasicircle. As was noted in Example 4.2 , the boundary $\Gamma$ of a quasidisk can be arbitrarily rough [GeVa] (as measured by means of the Hausdorff dimension).

Of course, for the Dirichlet Laplacian, our results apply to any of the above open sets, since no restriction (besides boundedness) is then imposed upon $\Omega$. Moreover, still for the Dirichlet Laplacian, they also apply to the (planar) "combs" considered in [Mt3, §VII.1, pp. 200-204]; recall that some of the latter ones were used in [Mt3] to show that Weyl's asymptotic formula (2.17) need not hold for the Neumann Laplacian. It is then natural to wonder whether, for the Neumann problem, part of our results (and methods) can be extended to include those "combs" for which the Neumann spectrum is discrete but does not behave according to Weyl's classical asymptotic formula. We hope to investigate this question in a later work.

We close $\S 5.1$ by stating the following two problems:

Problems. (a) For the Neumann Laplacian, find an alternative proof-based either on probabilistic or on heat equation methods-of our remainder estimate for the partition function $Z(t)$, obtained in the "fractal" case in Theorem 2.2 (or 4.1). (Recall that in this case, $\Omega$ is assumed to satisfy either the " $\left(C^{\prime}\right)$ condition" or the "extension property".) 
(b) A related although certainly more challenging problem is to attempt using wave equation methods in order to obtain refined spectral information about $\Omega$.

5.2. Modified Weyl-Berry conjecture. Recall from Definition 2.1 that $\Gamma$ is said to be Minkowski measurable (or "contented"), relative to $\Omega$, if

$$
0<\widetilde{\mathscr{M}}_{\widetilde{D}}(\Gamma)<+\infty \quad \text { and } \quad \widetilde{\mathscr{M}}_{\widetilde{D}}(\Gamma)=\lim _{\varepsilon \rightarrow 0^{+}} \varepsilon^{-(n-\widetilde{D})}\left|\widetilde{\Gamma}_{\varepsilon}\right|
$$

[Observe that we require here that a true limit-and not just an upper limitholds in (5.15).] Of course, $\widetilde{D}$ is the Minkowski dimension of $\Gamma$, relative to $\Omega$, and $\widetilde{\Gamma}_{\varepsilon}$ is given as in (2.5).

Similarly, $\Gamma$ is said to be Minkowski measurable (or "contented") if

$$
0<\mathscr{M}_{D}(\Gamma)<+\infty \text { and } \mathscr{M}_{D}(\Gamma)=\lim _{\varepsilon \rightarrow 0^{+}} \varepsilon^{-(n-D)}\left|\Gamma_{\varepsilon}\right|
$$

here, $D$ is the Minkowski dimension of $\Gamma$ and $\Gamma_{\varepsilon}$ is defined as in (2.1).

On the basis of this work and of the earlier work of Brossard and Carmona in [BrCa], we can now correct and refine in the following manner Berry's original conjecture $[\mathrm{Be} 1,2]$ for the asymptotic of the eigenvalues of the Laplacian on a bounded open set with "fractal" boundary:

Conjecture 5.1 (Modified Weyl-Berry conjecture). (i) [Dirichlet problem] Let $\Omega$ be a bounded open set of $\mathbb{R}^{n}(n \geq 1)$, with boundary $\Gamma$. Assume that $\Gamma$ is Minkowski measurable, relative to $\Omega$ (i.e., that (5.15) holds). Further, suppose that $\widetilde{D}$ belongs to the open interval $(n-1, n)$. Then, for the Dirichlet Laplacian, we have

$$
N(\lambda)=(2 \pi)^{-n} \mathscr{B}_{n}|\Omega|_{n} \lambda^{n / 2}-c_{n, \widetilde{D}} \widetilde{\mathscr{M}}_{\widetilde{D}}(\Gamma) \lambda^{\widetilde{D} / 2}+o\left(\lambda^{\widetilde{D} / 2}\right) \quad \text { as } \lambda \rightarrow+\infty,
$$

where $c_{n, \widetilde{D}}$ is a positive constant depending only on $n$ and $\widetilde{D}$.

(ii) [Neumann problem] Let $\Omega$ be a bounded open set of $\mathbb{R}^{n}(n \geq 2)$, with boundary $\Gamma$, satisfying either the " $\left(C^{\prime}\right)$ condition" or the "extension property". (See Definition 2.2 or 4.3.) Assume, in addition, that $\Gamma$ is Minkowski measurable (i.e., that $\left(5.15^{\prime}\right)$ holds). Further, suppose that $D$ belongs to the open interval $(n-1, n)$. Then, for the Neumann Laplacian, we have

$$
\left(5.16^{\prime}\right) \quad N(\lambda)=(2 \pi)^{-n} \mathscr{B}_{n}|\Omega|_{n} \lambda^{n / 2}+c_{n, D^{\prime}} \mathscr{M}_{D}(\Gamma) \lambda^{D / 2}+o\left(\lambda^{D / 2}\right) \quad \text { as } \lambda \rightarrow+\infty \text {, }
$$

where $c_{n, D}^{\prime}$ is a positive constant depending only on $n$ and $D$. [When $n=1$, we conjecture that $\left(D=\widetilde{D}\right.$ and) estimate $(5.16)$ [rather than $\left(5.16^{\prime}\right)$ ] holds in case (ii).]

Remarks 5.7. (a) We stress that we deal here-as we have done throughout this work-with the variational Dirichlet (resp., Neumann) problem in case (i) [resp., (ii)]. Moreover, since in case (i) [resp., (ii)], $\widetilde{D}>n-1$ (resp., $D>n-1)$, the boundary $\Gamma$ is assumed to be "fractal". 
(b) [Conjecture 5.1 $1^{\prime}$ Let $\phi(\lambda)=(2 \pi)^{-n} \mathscr{B}_{n}|\Omega|_{n} \lambda^{n / 2}$. Then, in case (i) [resp., (ii)], a different form of Conjecture 5.1 would consist in replacing (5.16) [resp., $\left(5.16^{\prime}\right)$ ] by the weaker conclusion

$$
\left.\phi(\lambda)-N(\lambda) \approx \lambda^{\tilde{D} / 2} \quad \text { [resp., } N(\lambda)-\phi(\lambda) \approx \lambda^{D / 2}\right] \text { as } \lambda \rightarrow+\infty,
$$

and the hypothesis of "Minkowski measurability" by the weaker assumption $0<\widetilde{\mathscr{M}}_{\widetilde{D}_{*}} \leq \widetilde{\mathscr{M}}_{\widetilde{D}}<+\infty$ (resp., $\left.0<\mathscr{M}_{D_{*}} \leq \mathscr{M}_{D}<+\infty\right)$. [Here, $\mathscr{M}_{D_{*} \text { denotes }}$ the lower Minkowski content of $\Gamma$, defined by taking the lower (rather than the upper) limit in (2.3); and similarly for $\widetilde{\mathscr{M}}_{\widetilde{D}_{*}}$. Of course, $\mathscr{M}_{D}=: \mathscr{M}_{D}^{*}$ still denotes the upper Minkowski content of $\Gamma$.] Our results and methods of proof (as well as Examples 5.1-5.1 ${ }^{\prime}$ and 5.2) provide good evidence for that.

For simplicity, we have limited ourselves to the Laplacian in the above statements; however, in view of Corollaries 2.1 and 2.2, Conjecture 5.1 ${ }^{\prime}$ can easily be extended to the more general elliptic operators of order $2 m$ considered in this paper. [Set $\phi(\lambda)=\mu_{\mathscr{\&}}^{\prime}(\Omega) \lambda^{n / 2 m}$, with $\mu_{\mathscr{A}}^{\prime}(\Omega)$ as in (2.18), and substitute in the conclusion $\lambda^{d / 2 m}$ for $\lambda^{d / 2}$, with $d=\widetilde{D}$ or $D$, respectively, further, assume that hypotheses (2.10) and (2.11) hold, with $V=H_{0}^{m}(\Omega)$ or $H^{m}(\Omega)$, for the Dirichlet or the Neumann problem, respectively.] The case of the pluriharmonic operator $\mathscr{A}:=(-\Delta)^{m} \quad(m \geq 1)$ is of particular interest.

(c) As a preliminary step, one can also consider the pre-Tauberian form of Conjectures 5.1 and 5.1'-in which the estimate for the "counting function" $N(\lambda)$ is replaced by the corresponding one for the "partition function" $Z(t)$. (For the Dirichlet Laplacian and under certain assumptions, aspects of this problem are examined in [ $\mathrm{BrCa}, \S 3]$.)

(d) The examples studied in $\S 5.1$ provide us with a variety of situations in which to test the modified Weyl-Berry conjecture, as well as its weaker forms mentioned in (b) and (c). (See especially Examples 5.1 and 5.1 ${ }^{\prime}$, in conjunction with Remark 5.2(c).)

(e) In the statement of Conjectures 5.1 and $5.1^{\prime}$, we may assume more generally that (instead of being bounded) $\Omega$ has finite volume. Indeed, all the results of this paper remain valid for such open sets.

In the present work, we have obtained a partial resolution of the above modified Weyl-Berry conjecture; in the process, we have learned new facts and developed new intuition about this and related problems. Hence, further attempts to verify (or disprove) this conjecture should lead to an even better understanding of the relationships between spectral and "fractal" geometry.

\section{APPENDIX A: AsYMPTOTICS OF THE PARTITION FUNCTION}

We show here how to derive Theorem 2.2 from Theorem 2.1 and its corollaries. To do so, we shall need the following simple refinement of the classical Abelian theorem. [This result is surely known but since we could not find a precise reference for it, we include a proof below. The classical Abelian theorem, as stated, for example, in [Si, Theorem 10.2, p. 107], corresponds to case (ii) with $\delta=\gamma$.] 
Theorem A (Abelian theorem with remainder). Let $\nu$ be a positive Borel measure on $[0,+\infty)$ that is concentrated on $\left[\lambda_{1},+\infty\right)$, for some $\lambda_{1}>0$. Set $F(\lambda)=\nu([0, \lambda))$, for $\lambda>0$.

(i) Assume that for some real constants $L, \gamma$, and $\delta$ with $0 \leq \delta \leq \gamma$,

$$
F(\lambda)=L \lambda^{\gamma}+O\left(\lambda^{\delta}\right) \text { as } \lambda \rightarrow+\infty .
$$

Then, if $G(t):=\int_{0}^{\infty} e^{-t x} d \nu(x)$, for $t \geq 0$, we have

$$
G(t)=L \gamma ! t^{-\gamma}+O\left(t^{-\delta}\right) \text { as } t \rightarrow 0^{+} .
$$

(ii) Moreover, this theorem still holds if " $O$ " is replaced by " $O$ " both in hypothesis (A-1) and the conclusion (A-2).

Proof of Theorem 2.2. Let $\nu:=\sum_{i=1}^{\infty} \delta_{\lambda_{i}}$, where $\left\{\lambda_{i}\right\}_{i=1}^{\infty}$ is the sequence of (positive) eigenvalues of $\left(\mathrm{P}_{\mathscr{A}}\right)$, repeated according to multiplicity and written in increasing order, as in (2.14); here, $\delta_{\alpha}$ denotes the Dirac measure at point $\alpha$. Obviously, $\nu$ is concentrated on $\left[\lambda_{1},+\infty\right)$. Further, $F(\lambda)=\nu([0, \lambda])=$ $\sum_{\lambda_{i} \leq \lambda} 1=N(\lambda)$, the "counting function" of $\left(\mathrm{P}_{\mathscr{A}}\right)$. Hence $G(t)=\int_{0}^{\infty} e^{-t x} d \nu(x)$ $=\sum_{i=1}^{\infty} e^{-\lambda_{i} t}$, the "partition function" associated with $\left(\mathrm{P}_{\mathscr{A}}\right)$. In view of Theorem A, the conclusion of Theorem 2.2 follows from Corollaries 2.1-2.2. Note that in this case, $L:=\mu_{\mathscr{A}}^{\prime}(\Omega), \gamma:=n / 2 m$, and $\delta:=d / 2 m$.

Proof of Theorem A. (i) By (Lebesgue-Stieltjes) integration by parts, $G(t)=$ $t \int_{0}^{\infty} e^{-t x} F(x) d x=\int_{0}^{\infty} e^{-y} F(y / t) d y$. Set $\varepsilon(\lambda)=\lambda^{-\delta}\left(F(\lambda)-L \lambda^{\gamma}\right)$, for $\lambda>0$, so that $F(\lambda)=L \lambda^{\gamma}+\lambda^{\delta} \varepsilon(\lambda)$. Then, since $\gamma !=\int_{0}^{\infty} e^{-y} y^{\gamma} d y$, we have

$$
G(t)=L \gamma ! t^{-\gamma}+t^{-\delta} \eta(t),
$$

where $\eta(t):=\int_{0}^{\infty} e^{-y} y^{\delta} \varepsilon(y / t) d y$, for $t>0$. Next, we claim that the function $\varepsilon$ is bounded on $(0,+\infty)$. [Indeed, since $F(\lambda)=0$ for $\lambda<\lambda_{1}, \varepsilon(\lambda)=-L \lambda^{\gamma-\delta}$ for $\lambda<\lambda_{1}$ (with $\gamma-\delta \geq 0$ ) and thus $\varepsilon$ is bounded near 0 ; further, $\varepsilon$ is bounded on every compact interval $[a, b] \subset(0,+\infty)$ since $F$ is nondecreasing. Hence the claim since, by assumption, $\varepsilon$ is bounded near $+\infty$.] We now deduce from the claim that $\|\eta\|_{L^{\infty}} \leq \delta !\|\varepsilon\|_{L^{\infty}}<+\infty$ and hence (A-3) yields (A-2).

(ii) In this case, we know in addition that $\varepsilon(\lambda) \rightarrow 0$, as $\lambda \rightarrow+\infty$. Then the conclusion follows from the claim and the dominated convergence theorem.

\section{Appendix B: Perturbation lemma}

We establish here Lemma 4.4 that was used in the proof of Proposition 4.7.

Let $V$ denote $H_{0}^{m}(\Omega)$ or $H^{m}(\Omega)$ according to whether we work with the Dirichlet or the Neumann problem, respectively, as in §4.2.A or 4.2.B. We proceed as in [Mt3, pp. 178-179 or FlLa2, pp. 352-353] but also take into account our simpler hypotheses on $\mathscr{A}$. We deduce from the interpolation inequalities (see, e.g., [Ag, p. 24]) that there exists a positive constant $c_{1}$ such that for all 
$\tau \in(0,1)$ and $u \in V$, we have $\left|a(u, u)-a^{\prime}(u, u)\right| \leq\left(\tau+\frac{1}{2}\right) a(u, u)+t\|u\|_{L^{2}(\Omega)}^{2}$, where $t:=c_{1}\left(\tau^{1-2 m}+1\right)$; here, $a^{\prime}$ is the leading form associated with $a$. It then follows from Proposition 4.2(ii) that $N\left(\lambda^{\prime} ; a^{\prime}\right) \leq N(\lambda ; a) \leq N\left(\lambda^{\prime \prime} ; a^{\prime}\right)$, with $\lambda^{\prime}:=\lambda\left(\frac{1}{2}-\tau\right)-t$ and $\lambda^{\prime \prime}:=\left(\frac{3}{2}+\tau\right)(\lambda+t)$; naturally, $N(\lambda ; a)$ or $N\left(\lambda ; a^{\prime}\right)$ refers here to the "counting function" associated with the operator $\mathscr{A}$ or $\mathscr{A}^{\prime}$, respectively, on all of $\Omega$.

Next, choose $\tau=c \lambda^{-1 / 2 m}$ and $\lambda \geq c^{\prime}\left(\varepsilon_{p}\right)^{-2 m}$ (with $c^{\prime}$ and hence $\lambda$ large enough); then $\lambda \geq c^{\prime \prime}\left[\left(\varepsilon_{p}\right)^{-2 m}+\tau^{-2 m}\right]$; further, $c_{1}^{\prime} \lambda \leq \lambda^{\prime} \leq \lambda \leq \lambda^{\prime \prime} \leq c_{2}^{\prime} \lambda$ and $0 \leq\left(\lambda^{\prime \prime}\right)^{n / 2 m}-\lambda^{n / 2 m} \leq C\left[\lambda^{n / 2 m}+\lambda^{(n-1) / 2 m}\right]$. Since clearly, by (2.18), $\mu_{\mathscr{A}^{\prime}}^{\prime}(\Omega)=\mu_{\mathscr{A}}^{\prime}(\Omega)$, we can now conclude the proof of Lemma 4.4 by applying Proposition 4.7 to $\mathscr{A}^{\prime}$ and choosing $p$ sufficiently large.

\section{Appendix C: Computation of Minkowski content}

We show here, in particular, how to compute the (relative) Minkowski content $\widetilde{\mathscr{M}}_{\widetilde{D}}(\Gamma)$ in Examples 5.1 and $5.1^{\prime}$. We note that in these examples, all we really needed to know was that $0<\widetilde{\mathscr{M}}_{\widetilde{D}}(\Gamma)<+\infty$; however, the more precise information obtained here should be useful in later work on the subject.

Theorem C. Given $a>0$, let $\Omega=\bigcup_{i=1}^{\infty} I_{i} \subset \mathbb{R}$, with $I_{i}=\left((i+1)^{-a}, i^{-a}\right)$, for all $i \geq 1$, as in Example 5.1. Then $\Gamma:=\partial \Omega$ is Minkowski measurable, relative to $\Omega$ (in the sense of Definition 2.1) and we have

$$
\widetilde{D}=\widetilde{D}(\Gamma)=\frac{1}{a+1} \quad \text { and } \quad \widetilde{\mathscr{M}}_{\widetilde{D}}(\Gamma)=2^{a /(a+1)}\left(a^{1 /(a+1)}+a^{-a /(a+1)}\right)=\frac{2^{1-\widetilde{D}}}{1-\widetilde{D}} a^{\widetilde{D}}
$$

Proof. For $i \geq 1$, let $l_{i}=b_{i}-b_{i+1}$ denote the length of $I_{i}$, with $b_{i}:=i^{-a}$. Fix $\varepsilon>0$ less than $1 / 4$. Clearly, $\widetilde{\Gamma}_{\varepsilon}=\bigcup_{i=1}^{\infty}\left(\widetilde{\Gamma}_{i}\right)_{\varepsilon}$ and for each $i \geq 1,\left|\left(\widetilde{\Gamma}_{i}\right)_{\varepsilon}\right|=$ $\min \left(l_{i}, 2 \varepsilon\right)$. [Here, $|\cdot|$ stands for $|\cdot|_{1}$ and $\Gamma_{i}$ denotes the boundary of $I_{i}$. Further, we work implicitly with $A:=\Gamma \backslash\{0\}=\left\{j^{-a}: j=1,2, \ldots\right\}$ in place of $\Gamma$; this does not affect the outcome since $\Gamma=\bar{A}$ and hence $\widetilde{D}=\widetilde{D}(A)$ and $\widetilde{\mathscr{M}}_{\widetilde{D}}(\Gamma)=\widetilde{\mathscr{M}}_{\widetilde{D}}(A)$.] Thus, if we let

$$
i_{0}=i_{0}(\varepsilon)=\min \left\{i \geq 1: \varepsilon \geq l_{i} / 2\right\}-1 \text {, }
$$

we have

$$
\left|\widetilde{\Gamma}_{\varepsilon}\right|=\sum_{i=1}^{\infty}\left|\left(\widetilde{\Gamma}_{i}\right)_{\varepsilon}\right|=2 \varepsilon i_{0}(\varepsilon)+\sum_{i=i_{0}(\varepsilon)+1}^{\infty} l_{i} .
$$

Since $\sum_{i=i_{0}(\varepsilon)+1}^{\infty} l_{i}=b_{i_{0}(\varepsilon)+1}$, it follows from $(\mathrm{C}-3)$ that for $0 \leq d \leq n:=1$,

$$
L_{d}(\varepsilon):=\varepsilon^{-(n-d)}\left|\widetilde{\Gamma}_{\varepsilon}\right|=\varepsilon^{d}\left(\varepsilon^{-1}\left|\widetilde{\Gamma}_{\varepsilon}\right|\right)=\varepsilon^{d}\left(2 i_{0}(\varepsilon)\right)+\varepsilon^{-(1-d)} b_{i_{0}(\varepsilon)+1} .
$$

According to (C-4) and Definition 2.1, we have

$$
\widetilde{D}=\inf \left\{d \geq 0: \lim _{\varepsilon \rightarrow 0^{+}} L_{d}(\varepsilon)=0\right\} \quad \text { and } \quad \widetilde{\mathscr{M}}_{\widetilde{D}}(\Gamma)=\limsup _{\varepsilon \rightarrow 0^{+}} L_{\widetilde{D}}(\varepsilon) .
$$


Now, in the present case, $l_{i}=i^{-a}-(i+1)^{-a} \sim a i^{-(a+1)}$, as $i \rightarrow \infty$; hence, as $\varepsilon \rightarrow 0^{+}, i_{0}(\varepsilon) \sim(2 \varepsilon / a)^{-1 /(a+1)}$ and also, $b_{i_{0}(\varepsilon)+1}=\left(i_{0}(\varepsilon)+1\right)^{-a} \sim(2 \varepsilon / a)^{a /(a+1)}$. Consequently,

$$
\begin{gathered}
\varepsilon^{d}\left(2 i_{0}(\varepsilon)\right) \sim 2^{a /(a+1)} a^{1 /(a+1)} \varepsilon^{d-1 /(a+1)}, \\
\varepsilon^{-(1-d)} b_{i_{0}(\varepsilon)+1} \sim 2^{a /(a+1)} a^{-1 /(a+1)} \varepsilon^{d-1 /(a+1)},
\end{gathered}
$$

as $\varepsilon \rightarrow 0^{+}$. [We note that in the "midfractal" case when $a=1$, the right-hand sides of (C-6) coincide.] Thus, by (C-4) and (C-6), we have as $\varepsilon \rightarrow 0^{+}$,

$$
L_{d}(\varepsilon) \sim 2^{a /(a+1)}\left(a^{1 /(a+1)}+a^{-a /(a+1)}\right) \varepsilon^{d-1 /(a+1)} .
$$

In light of (C-4), (C-5), and (C-7), we conclude that $\widetilde{D}=1 /(a+1)$ and

$$
\widetilde{\mathscr{M}}_{\widetilde{D}}(\Gamma)=\lim _{\varepsilon \rightarrow 0^{+}} L_{\widetilde{D}}(\varepsilon)=2^{a /(a+1)}\left(a^{1 /(a+1)}+a^{-a /(a+1)}\right)=\frac{2^{1-\widetilde{D}}}{1-\widetilde{D}} a^{\widetilde{D}},
$$

as desired; in particular, $\Gamma$ is Minkowski measurable, relative to $\Omega$, since the limit in (C-8) exists and belongs to $(0,+\infty)$.

Remarks C. (a) An entirely similar-although somewhat more involved-computation shows that in Example 5.1 $, \widetilde{D}=(n-1)+(a+1)^{-1}, \Gamma$ is Minkowski measurable, and $\widetilde{\mathscr{M}}_{\widetilde{D}}(\Gamma)$ is still given by the right side of $(\mathrm{C}-1)$.

(b) According to Remark 5.1(a), we have $\widetilde{D}=D$ in Example 5.1 (as well as $\left.5.1^{\prime}\right)$. Of course, this fact can also be deduced from a direct calculation analogous to the above one; the latter would also show that $\mathscr{M}_{D}(\Gamma)=\widetilde{\mathscr{M}}_{\widetilde{D}}(\Gamma)$ in this case.

(c) In Example $5.1^{\prime}$ where $n \geq 1$, we can justify the fact that $H=H(\Gamma)=$ $n-1$ as follows: clearly, $\Gamma=\bigcup_{i=1}^{\infty} A_{i}$, with $A_{i}:=\left\{q_{i}\right\} \times(0,1)^{n-1}$ for $i \geq 0$; here, $q_{i}:=i^{-a}$ (resp., $=0$ ) if $i \geq 1$ (resp., $=0$ ). Since obviously, $H\left(A_{i}\right) \equiv$ $n-1$, it thus follows from the properties of the Hausdorff dimension [see $\S 3$ and Remarks 5:2(a),(b)] that $H=\sup _{i \geq 0} H\left(A_{i}\right)=n-1$.

Note added in proof. We indicate here some recent results obtained since this paper was written. In the one-dimensional case (i.e., when $n=1$ ), we have now established the "modified Weyl-Berry conjecture" (Conjecture 5.1) and obtained in the process some unexpected and intriguing connections with the Riemann zeta-function. As was suggested in particular in Remark 5.7(d) above, Example 5.1 played a key role in this situation. (See [1], M. L. Lapidus and C. Pomerance, The Riemann zeta-function and the one-dimensional Weyl-Berry conjecture for fractal drums, preprint, 1990, announced in [2], M. L. Lapidus and C. Pomerance, Fonction zêta de Riemann et conjecture de Weyl-Berry pour les tambours fractals, C. R. Acad. Sci. Paris Sér. I. Math. 310 (1990), 343-348.) Moreover, when $n \geq 2$, Conjecture 5.1 was disproved in [3], M. L. Lapidus and C. Pomerance, Epstein zeta-functions and the n-dimensional Weyl-Berry conjecture for fractal drums (in preparation), and a further refinement of (the conclusion 
of) Conjecture 5.1 was proposed in [3] and [4], M. L. Lapidus, Spectral and fractal geometry: from the Weyl-Berry conjecture for the vibrations of fractal drums to the Riemann zeta-function, Proc. UAB International Conference on Mathematical Physics and Differential Equations (Birmingham, March, 1990), (C. Bennewitz, et al., eds.), Academic Press, New York, 1990, pp. 171-201 (in press). This new version of Conjecture 5.1 is expressed in terms of the "spectral zeta-function," which is further studied in [5], M. L. Lapidus, Spectral zeta-functions, vibrating fractal strings and the Dirichlet divisor problem (in preparation), and in [3]. In [1], the authors have also proved Conjecture $5.1^{\prime}$ as well as its converse when $n=1$. A suitable form of the converse of Conjecture 5.1 when $n=1$ has been shown to be closely connected with the Riemann hypothesis in [6], M. L. Lapidus and H. Maier, The Riemann hypothesis, vibrating fractal strings and the modified Weyl-Berry conjecture, to be announced in [7], M. L. Lapidus and H. Maier, Hypothèse de Riemann, cordes fractales vibrantes et conjecture de Weyl-Berry modifiée, C. R. Acad. Sci. Paris Sér. I Math. (to appear).

Finally, we point out that explicit examples when the Minkowski dimensions $D$ and $\widetilde{D}$ differ for the boundary $\Gamma=\partial \Omega$ of a bounded open set, are given in [7], C. Tricot, Dimensions aux bords d'un ouvert, Ann. Sci. Math. Québec 11 (1987), 205-235.

The author wishes to thank Professor Elliott H. Lieb for a thoughtful comment.

He is also grateful to the National Science Foundation for continued support through Grant DMS-8904389, as well as to the University of Georgia for the M. G. Michael Award for Excellence in Research from the Franklin College of Arts and Sciences.

\section{ACKNOWLEDGMENTS}

I am indebted to the National Science Foundation for supporting this research through Grant DMS-8703138. I also wish to acknowledge the support of the CNPq and the Organization of Latin American States at the Instituto de Matemática Pura E Aplicada (IMPA) in Rio de Janeiro, Brazil, as well as the Université Pierre et Marie Curie (Paris VI) and the Université Claude Bernard (Lyon I) in France.

I particularly wish to thank Professor Raphael José Iorio, Jr., for his hospitality during my stay at IMPA and Professor Jean-Bernard Baillon for his invitation to the Universite Claude Bernard, where the beginning of this paper was written during the Summer of 1987 while the author was a Professeur Associé.

This work was presented at the Special Session on "Multidimensional Inverse Problems, Related Problems in Analysis and Applications" at the Annual Meeting of the American Mathematical Society held in Atlanta, Georgia, in January 1988. (Abstract \#839-35-103.) I am also grateful to the organizers of these conferences and to the following institutions, where the main results of 
the paper were presented: the Universite Pierre et Marie Curie in Paris (June 1987), the Centre de Physique Théorique (CNRS, Luminy) of the Universite Aix-Marseille (July 1987), the Conference on "Harmonic Analysis and Related Areas" held at Auburn University, Alabama (November 1987), Boston College (February 1988), the "International Conference on Theory and Applications of Differential Equations" held in Columbus, Ohio (March 1988), as well as the Conference on "Harmonic Analysis and its Applications" and the Workshop on "Differential Geometry, Calculus of Variations, and Computer Graphics," both held at the Mathematical Sciences Research Institute (MSRI) in Berkeley, California (March and May 1988).

Finally, I wish to thank Mrs. Tilda Runner and Ms. Gail Suggs for their patient and diligent typing of the manuscript.

\section{REFERENCES}

[Ad] R. A. Adams, Sobolev spaces, Academic Press, New York, 1975.

[Ag] S. Agmon, Lectures on elliptic boundary value problems, Van Nostrand, Princeton, N. J., 1965.

[Al] F. J. Almgren, Jr., Plateau's problem: an invitation to varifold geometry, Benjamin, New York, 1966.

[BaHi] H. P. Baltes and E. R. Hilf, Spectra of finite systems, B. I.-Wissenschaftsverlag, Vienna, 1976.

[Bd] P. H. Bérard, Remarques sur la conjecture de Weyl, Compositio Math. 48 (1983), 35-53.

[Be1] M. V. Berry, Distribution of modes in fractal resonators, Structural Stability in Physics (W. Güttinger and H. Eikemeier, eds.), Springer-Verlag, Berlin, 1979, pp. 51-53.

[Be2] _ Some geometric aspects of wave motion: wavefront dislocations, diffraction catastrophes, diffractals, Geometry of the Laplace Operator, Proc. Sympos. Pure Math., vol. 36, Amer. Math. Soc., Providence, R. I., 1980, pp. 13-38.

[BiSo] M. S. Birman and M. Z. Solomjak, Piecewise polynomial approximation of functions of the class $W_{p}^{\alpha}$, Math. USSR-Sb. 2 (1967), 295-317.

[Bo] G. Bouligand, Ensembles impropres et nombre dimensionnel, Bull. Sci. Math. (2) 52 (1928), 320-344 and 361-376.

[BG] L. Boutet de Monvel and P. Grisvard, Le comportement asymptotique des valeurs propres d'un opérateur, C.R. Acad. Sci. Paris Sér. A 272 (1971), 23-25.

[BrCa] J. Brossard and R. Carmona, Can one hear the dimension of a fractal?, Comm. Math. Phys. 104 (1986), 103-122.

[C] I. Chavel, Eigenvalues in Riemannian geometry, Academic Press, Orlando, Fla., 1984.

[Ce] G. Cherbit (ed.), Fractals, dimensions non entieres et applications, Masson, Paris, 1987.

[Ch] G. Choquet, Outils topologiques et métriques de l'analyse mathématique, Centre de Documentation Universitaire, Paris, 1969.

[Cn] D. L. Cohn, Measure theory, Birkhäuser, Boston, Mass., 1980.

[Co] R. Courant, Über die Eigenwerte bei den Differentialgleichungen der mathematischen Physik, Math. Z. 7 (1920), 1-57.

[CoHi] R. Courant and D. Hilbert, Methods of mathematical physics, Vol. I, English transl., Interscience, New York, 1953.

[DMT] Y. Dupain, M. Mendès-France, and C. Tricot, Dimension des spirales, Bull. Soc. Math. France 111 (1983), 193-201.

[EdEv] D. E. Edmunds and W. D. Evans, Spectral theory and differential operators, Oxford Univ. Press, Oxford, 1987. 
[Ek] A. El Kolli, Nième épaisseur dans les espaces de Sobolev, J. Approx. Theory 10 (1974), 268-294.

[Fa] W. Falconer, The geometry of fractal sets, Cambridge Univ. Press, London, 1985.

[Fe] H. Federer, Geometric measure theory, Springer, New York, 1969.

[Ff] C. L. Fefferman, The uncertainty principle, Bull. Amer. Math. Soc. (N.S.) 9 (1983), 129206.

[FILa1] J. Fleckinger and M. L. Lapidus, Eigenvalues of elliptic boundary value problems with an indefinite weight function, Trans. Amer. Math. Soc. 295 (1986), 305-324.

[FlLa2] __ Remainder estimates for the asymptotics of elliptic eigenvalue problems with indefinite weights, Arch. Rational Mech. Anal. 98 (1987), 329-356.

[FlMt] J. Fleckinger and G. Métivier, Théorie spectrale des opérateurs uniformément elliptiques sur quelques ouverts irréguliers, C.R. Acad. Sci. Paris Sér. A 276 (1973), 913-916.

[GeVa] F. W. Gehring and J. Väisälä, Hausdorff dimension and quasiconformal mappings, J. London Math. Soc. 6 (1973), 504-512.

[Gi] P. B. Gilkey, Invariance theory, the heat equation, and the Atiyah-Singer index theorem, 2nd ed., Publish or Perish, Wilmington, Del., 1984.

[Gr] D. Gromes, Über die asymptotische Verteilung der Eigenwerte des Laplace Operators für Gebiete auf der Kugeloberfläche, Math. Z. 94 (1966), 110-121.

[GuKz] V. W. Guillemin and D. Kazhdan, Some inverse spectral results for negatively curved 2manifolds, Topology 19 (1980), 301-312.

[Ha] J. Hawkes, Hausdorff measure, entropy, and the independence of small sets, Proc. London Math. Soc. (3) 28 (1974), 700-724.

[Ho1] L. Hörmander, The spectral function of an elliptic operator, Acta Math. 121 (1968), 193-218.

[Ho2] _ The analysis of linear partial differential operators, Vols. III and IV, Springer-Verlag, Berlin, 1985.

[HrWa] W. Hurewicz and H. Wallman, Dimension theory, Princeton Univ. Press, Princeton, N. J., 1941.

[Hu] J. E. Hutchinson, Fractals and self-similarity, Indiana Univ. Math. J. 30 (1981), 713-747.

[Iv1] V. Ja. Ivrii, Second term of the spectral asymptotic expansion of the Laplace-Beltrami operator on manifolds with boundary, Functional Anal. Appl. 14 (1980), 98-106.

[Iv2] _- Precise spectral asymptotics for elliptic operators acting in fiberings over manifolds with boundary, Lecture Notes in Math., vol. 1100, Springer-Verlag, Berlin, 1984.

[JhLa] G. W. Johnson and M. L. Lapidus, Generalized Dyson series, generalized Feynman diagrams, the Feynman integral and Feynman's operational calculus, Mem. Amer. Math. Soc. No. 351, 62 (1986), 1-78.

[Jn] P. W. Jones, Quasiconformal mappings and extendability of functions in Sobolev spaces, Acta Math. 147 (1981), 71-88.

[Ka] M. Kac, Can one hear the shape of a drum?, Amer. Math. Monthly (Slaught Memorial Papers, No. 11) (4) 73 (1966), 1-23.

[Kh] J.-P. Kahane, Courbes étranges, ensembles minces, Bull. Assoc. Professeurs Enseign. Math. Phys. 275/276 (1970), 325-339.

[KhSa] J.-P. Kahane and R. Salem, Ensembles parfaits et séries trigonométriques, Hermann, Paris, 1963.

[Ku] N. V. Kuznetsov, Asymptotic distribution of the eigenfrequencies of a plane membrane in the case when the variables can be separated, Differential Equations 2 (1966), 715-723.

[La1] M. L. Lapidus, Valeurs propres du laplacien avec un poids qui change de signe, C.R. Acad. Sci. Paris Sér. I Math. 298 (1984), 265-268.

[La2] _ Spectral theory of elliptic problems with indefinite weights, Spectral Theory of SturmLiouville Differential Operators (Hans G. Kaper and A. Zettle, eds.), ANL-84-73, Argonne National Laboratory, Argonne, 1984, pp. 159-168. 
[La3] _ Formules de Trotter et calcul opérationnel de Feynman, Thèse de Doctorat d'Etat ès Sciences, Mathématiques, Université Pierre et Marie Curie (Paris VI), France, 1986. (Part II: Problèmes aux valeurs propres elliptiques avec un poids non défini. Part III: Calcul opérationnel de Feynman.)

[La4] _ The differential equation for the Feynman-Kac formula with a Lebesgue-Stieltjes measure, Lett. Math. Phys. 11 (1986), 1-13. (Dedicated to the memory of Mark Kac.)

[La5] _ The Feynman-Kac formula with a Lebesgue-Stieltjes measure and Feynman's operational calculus, Stud. Appl. Math. 76 (1987), 93-132.

[La6] _ The Feynman-Kac formula with a Lebesgue-Stieltjes measure: an integral equation in the general case, Integral Equations Operator Theory 12 (1989), 163-210.

[La7] Strong product integration of measures and the Feynman-Kac formula with a LebesgueStieltjes measure, Suppl. Rend. Circ. Mat. Palermo Ser. II 17 (1987), 271-312.

[La8] _ Asymptotic distribution of the eigenvalues of elliptic boundary value problems and Schrödinger operators with indefinite weights, abridged version of a talk given at the VIIIth Latin American School of Mathematics on "Partial Differential Equations" (IMPA, Rio de Janeiro, Brazil, July 1986).

[La9] _ Can one hear the shape of a fractal drum? Partial resolution of the Weyl-Berry conjecture, Geometric Analysis and Computer Graphics (P. Concus et al., eds.), Proc. Workshop Differential Geometry, Calculus of Variations, and Computer Graphics (MSRI, Berkeley, May 1988), Mathematical Sciences Research Institute Publications, vol. 17, SpringerVerlag, New York, 1990, pp. 119-126.

[La10] _ Elliptic differential operators on fractals and the Weyl-Berry conjecture (in preparation).

[LaFl] M. L. Lapidus and J. Fleckinger-Pellé, Tambour fractal: vers une résolution de la conjecture de Weyl-Berry pour les valeurs propres du laplacien, C.R. Acad. Sci. Paris Sér. I Math. 306 (1988), 171-175.

[LiMa] J. L. Lions and E. Magenes, Non-homogeneous boundary value problems and applications, Vol. I, English transl., Springer-Verlag, Berlin, 1972.

[Lo] G. G. Lorentz, Approximation of functions, 2nd ed., Chelsea, New York, 1986.

[McSn] H. P. McKean and I. M. Singer, Curvature and the eigenvalues of the Laplacian, J. Differential Geom. 1 (1967), 43-69.

[Md1] B. B. Mandelbrot, The fractal geometry of nature, rev. and enlarged ed., Freeman, New York, 1983.

[Md2] _ Les objets fractals, 2nd ed., Flammarion, Paris, 1984.

[MrVu] O. Martio and M. Vuorinen, Whitney cubes, p-capacity, and Minkowski content, Exposition. Math. 5 (1987), 17-40.

[M] V. G. Maz'ja, Sobolev spaces, Springer-Verlag, Berlin, 1985.

[Ms1] R. B. Melrose, Weyl's conjecture for manifolds with concave boundary, Geometry of the Laplace Operator, Proc. Sympos. Pure Math., vol. 36, Amer. Math. Soc., Providence, R. I., 1980, pp. 254-274.

[Ms2] __, The trace of the wave group, Contemp. Math., vol. 27, Amer. Math. Soc., Providence, R. I., 1984, pp. 127-167.

[Mt1] G. Métivier, Théorie spectrale d'opérateurs elliptiques sur des ouverts irréguliers, Séminaire Goulaic-Schwartz, No. 21, Ecole Polytechnique, Paris, 1973.

[Mt2] _ Etude asymptotique des valeurs propres et de la fonction spectrale de problemes aux limites, Thèse de Doctorat d'Etat, Mathématiques, Université de Nice, France, 1976.

[Mt3] _ Valeurs propres de problèmes aux limites elliptiques irréguliers, Bull. Soc. Math. France Mém. 51-52 (1977), 125-219.

[OsWi] R. Osserman and A. Weinstein (eds.), Geometry of the Laplace operator, Proc. Sympos. Pure Math., vol. 36, Amer. Math. Soc., Providence, R. I., 1980.

[PeRi] H. O. Peitgen and P. H. Richter, The beauty of fractals, Springer-Verlag, Berlin, 1986. 
[Ph] Pham The Lai, Meilleures estimations asymptotiques des restes de la fonction spectrale et des valeurs propres relatifs au laplacien, Math. Scand. 48 (1981), 5-38.

[PiTo] L. Pietronero and M. Tozzati (eds.), Fractals in physics, North-Holland, Amsterdam, 1986.

[Pn] A. Pinkus, n-widths in approximation theory, Springer-Verlag, New York, 1985.

[Pr] M. H. Protter, Can one hear the shape of a drum? Revisited, SIAM Rev. 29 (1987), 185-197.

[ReSi] M. Reed and B. Simon, Methods of modern mathematical physics, Vol. IV, Analysis of Operators, Academic Press, New York, 1978.

[Ro] C. A. Rogers, Hausdorff measures, Cambridge Univ. Press, Cambridge, 1970.

[SMR] M. F. Schlesinger, B. B. Mandelbrot, and R. J. Rubin (eds.), Fractals in the physical sciences, J. Statist. Phys. 36 Nos. 5/6, (1984).

[Se1] R. T. Seeley, A sharp asymptotic remainder estimate for the eigenvalues of the Laplacian in a domain of $\mathbb{R}^{3}$, Adv. in Math. 29 (1978), 244-269.

[Se2] __ An estimate near the boundary for the spectral function of the Laplace operator, Amer. J. Math. 102 (1980), 869-902.

[Si] B. Simon, Functional integration and quantum physics, Academic Press, New York, 1979.

[Th] W. Thompson, d'Arcy, On growth and form, abridged ed. (J. T. Bonner, ed.), Cambridge Univ. Press, Cambridge, 1966.

[Tr1] C. Tricot, Jr., Douze définitions de la densité logarithmique, C.R. Acad. Sci. Paris Sér. I Math. 293 (1981), 549-552.

[Tr2] _ Two definitions of fractional dimension, Math. Proc. Cambridge Philos. Soc. 91 (1982), 57-74.

[Tr3] _ Metric properties of compact sets of measure zero in $\mathbb{R}^{2}$, preprint; in Mesures et Dimensions, Thèse de Doctorat d'Etat, Mathématiques, Université Paris-Sud (Orsay), France, 1983.

[Ur] H. Urakawa, Bounded domains which are isospectral but not congruent, Ann. Sci. Ecole Norm. Sup. (4) 15 (1982), 441-456.

[VGL] S. K. Vodopjanov, V. M. Gol'dstein, and T. G. Latfullin, Criteria for extension of functions of the class $L_{2}^{1}$ from unbounded plane domains, Siberian Math. J. 20 (1979), 298-301.

[Wb] H. F. Weinberger, Variational methods for eigenvalue approximation, CBMS Regional Conf. Ser. Appl. Math., vol. 15, SIAM, Philadelphia, Pa., 1974.

[We1] H. Weyl, Über die asymptotische verteilung der Eigenwerte, Gott. Nach. (1911), 110-117.

[We2] _ Das asymptotische Verteilungsgesetz der Eigenwerte linearer partieller Differentialgleichungen, Math. Ann. 71 (1912), 441-479.

[We3] _ Über die Abhängigkeit der Eigenschwingungen einer Membran von deren Begrenzung, J. Angew. Math. 141 (1912), 1-11.

[Ya] S.-T. Yau, Nonlinear analysis in geometry, Enseign. Math. (2) 33 (1987), 109-158.

Department of Mathematics, Yale University, 10 Hillhouse Avenue, Box 2155 Yale Station, New Haven, Connecticut 06520

Current address: Department of Mathematics, University of California, Riverside, California 92521-0135 